Portland State University

PDXScholar

Spring 5-22-2017

\title{
Why Do Animals Do What They Do, When They Do It? \\ Characterizing the Role of the Hypothalamus- \\ Pituitary-Adrenal Axis in Seasonal Life-History \\ Transitions
}

Catherine Anne Dayger Forbes

Portland State University

Follow this and additional works at: https://pdxscholar.library.pdx.edu/open_access_etds

Part of the Animal Sciences Commons, and the Biology Commons

Let us know how access to this document benefits you.

\section{Recommended Citation}

Dayger Forbes, Catherine Anne, "Why Do Animals Do What They Do, When They Do It? Characterizing the Role of the Hypothalamus-Pituitary-Adrenal Axis in Seasonal Life-History Transitions" (2017).

Dissertations and Theses. Paper 3614.

https://doi.org/10.15760/etd.5506

This Dissertation is brought to you for free and open access. It has been accepted for inclusion in Dissertations and Theses by an authorized administrator of PDXScholar. Please contact us if we can make this document more accessible: pdxscholar@pdx.edu. 
Why Do Animals Do What They Do, When They Do It?

Characterizing the Role of the Hypothalamus-Pituitary-Adrenal Axis in Seasonal Life-History Transitions

by

Catherine Anne Dayger

A dissertation submitted in partial fulfillment of the requirements for the degree of

Doctor of Philosophy

in

Biology

Dissertation Committee:

Deborah I. Lutterschmidt, Chair

Bradley Buckley

Suzanne Estes

Michael P. LeMaster

Michael T. Murphy

Natalie Vasey

Portland State University

2017 
(C) 2017 Catherine Anne Dayger 


\begin{abstract}
Resource availability follows seasonal cycles in environmental conditions. To align physiology and behavior with prevailing environmental conditions, seasonal animals integrate cues from the environment with their internal state. One of the systems animals use to integrate those cues is the hypothalamus-pituitary-adrenal (HPA) axis and its primary effector, glucocorticoid hormones. The HPA axis has wide-ranging effects on physiology and behavior and, in the context of a glucocorticoid stress response, is known to mediate tradeoffs between immediate survival and future fitness. The HPA axis also plays an important role in facilitating predictable life-history events. Variation in HPA axis activity has been reported in all vertebrates, often coordinating seasonal reproduction and possibly also transitions between life-history stages. My dissertation research used red-sided garter snakes (Thamnophis sirtalis parietalis) to examine the role of the HPA axis in regulating seasonal life-history transitions, especially in females

In Chapter 2, I hypothesized that seasonal plasticity in stress responses is regulated, in part, by changes in the responsiveness of the adrenal glands to adrenocorticotropic hormone (ACTH). I found that glucocorticoid responses to ACTH challenge were smaller in males than in females during the spring, suggesting that reports of reduced stress responsiveness in males may reflect lower adrenal responsiveness to ACTH. The sex difference in mating season duration and consequently also in the timing of migration led me to hypothesize that sex differences in HPA axis activity could explain sex differences in the timing of migration. Furthermore, adrenal responsiveness to ACTH also varied seasonally in males, but not females, suggesting that female stress
\end{abstract}


responses, which have not been studied, may not vary seasonally.

In Chapter 3, I addressed this gap in our knowledge and investigated potential seasonal variation in female stress responses. In males, baseline glucocorticoids decrease over the course of the mating season resulting in significantly lower baseline levels in males that have begun to migrate. I hypothesized that a change in HPA axis activity occurs during spring and fall migration. Peak stress-induced glucocorticoid concentration occurred at an earlier sampling time in females during the spring compared to the fall. Peak stress-induced glucocorticoid concentrations also occurred at a later sampling time in migrating females than in pre-migratory females during the spring, suggesting that negative feedback regulation of the HPA axis changes as soon as females begin to migrate during the spring.

Female red-sided garter snake populations found in Manitoba are biennial breeders that give birth approximately every other year implying that a female's recent reproductive history can influence whether or not she will reproduce in a given year. Body condition can be used as a proxy for recent reproductive history and can be related to baseline and stress-induced glucocorticoid concentrations. In Chapter 4, I hypothesized that hormonal and behavioral stress responses vary with body condition. Baseline glucocorticoids did not vary with body condition, but females in low body condition showed a significantly larger increase in plasma glucocorticoids in response to capture stress. Body condition, but not capture stress, influenced latency to copulate, suggesting that females are resistant to the behavioral effects of capture stress during the spring mating season. Only females in low body condition increased latency to copulate in 
response to injection of a physiological $(15 \mu \mathrm{g})$ dose of exogenous CORT, while all females responded to a pharmacological $(60 \mu \mathrm{g})$ dose, indicating that behavioral responses to exogenous glucocorticoids vary with female body condition. These data suggest that variation in body condition may be associated with differences in HPA axis sensitivity and/or glucocorticoid receptor (GR) density in the brain.

I directly tested if there is a relationship among body condition, reproductive history and HPA axis activity in Chapter 5. I found that glucocorticoid stress responses and mating behavior did not vary with body condition, nor was body condition related to brain GR or reproductive condition (parturient vs post-parturient females). Only unreceptive females showed a significant stress-induced increase in glucocorticoids, suggesting that reduced stress responsiveness is associated with receptivity. Parturient females mated faster (were more proceptive) than post-parturient females. These data suggest that HPA axis activity influences reproductive "decisions" by modulating receptivity, while proceptivity is related primarily to recent reproductive history.

Together, these chapters help characterize how HPA axis activity varies with season, sex, reproductive history and migration status. By systematically probing the HPA axis in a single, tractable system, I have gained insight into how changes in the HPA axis support and modulate transitions between life-history stages. These results highlight the HPA axis' important function in mediating the critical trade-offs all animals must navigate to be successful in a changing world. 


\section{Dedication}

This, like all of my previous writing projects, is dedicated to my aunt, Mary Sullivan.

Aunt Mary was the wielder of a piquant wit, a verbal jujitsu master, an unconventional thinker, the bearer of a wicked sense of humor, and ceaseless lover of puns.

Aunt Mary was also a professor and poet who died with her PhD in creative writing incomplete.

This one's for you. 


\section{Acknowledgements}

With deepest gratitude, I thank my advisor Deborah Lutterschmidt for her thoughtful guidance and feedback during my time at Portland State University. I also thank my committee, Bradley Buckley, Suzanne Estes, Michael LeMaster, Michael T. Murphy, and Natalie Vasey for their help in shaping my course of study. Many thanks are due to my husband Scot Forbes has supported me through long hours in the lab and at the computer, and is excitedly planning the next adventure with me. I appreciate the help of the Lutterschmidt Lab: Deborah Lutterschmidt, Kim Dolan, Sharon Glaeser, Christina Howard, William Joersz, Ashley Lucas, Kalera Stratton, Raymond Whiteman, Rachel Wilson, and Treven Winters. Thanks also to Adrienne Godshalx for being my enthusiastic cheerleader, and for her help with my writing. I would like to thank my colleagues Kessina Lee, Monte Mattson, James Powell, Claire Riggs, and Kyle Tidwell for being a constant source of encouragement. I appreciate the help of Deb Duffield for encouraging me to learn more about science policy. My gratitude to Brad Buckley and Jason Podrabsky for generously sharing their expertise and equipment while I developed my Western blot assay for glucocorticoid receptor. Thanks to the Family Forbes for welcoming me without hesitation and celebrating my triumphs as if they were their own. Finally, thanks to my mom and dad, Anne de Velder and John Dayger, for shaping me into an independent thinker, for giving me a life-long appreciation for the arts, and especially for believing in me unflinchingly. 
Table of Contents

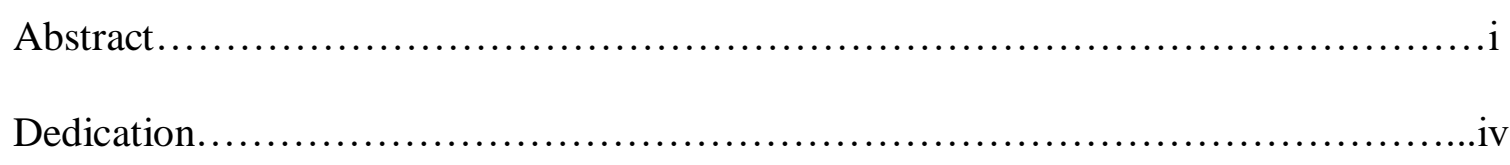

Acknowledgements......................................................

List of Tables............................................................

List of Figures......................................................... viii

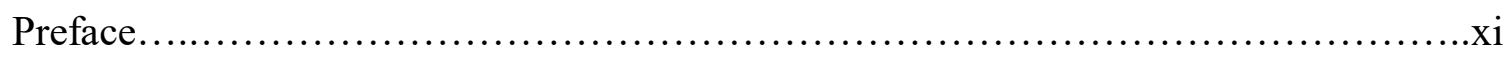

Chapter 1................................................................ Introduction

Chapter 2................................................................ 22

Seasonal and sex differences in responsiveness to adrenocorticotropic hormone contribute to stress response plasticity in red-sided garter snakes (Thamnophis sirtalis parietalis)

Chapter 3.

Patterns of stress responses shift during seasonal life-history transitions: an analysis comparing baseline, maximal and integrated corticosterone in female red-sided garter snakes (Thamnophis sirtalis parietalis).

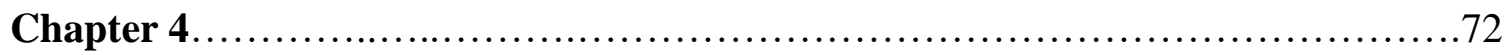

Responses to capture stress and exogenous corticosterone vary with body condition in female red-sided garter snakes (Thamnophis sirtalis parietalis)

Chapter 5

Physiological correlates of reproductive decisions: Relationships among body condition, reproductive status, and the hypothalamus-pituitary-adrenal axis in a reptile

Chapter 6.

Discussion and Conclusions

References. 


\section{List of Tables}

\section{Chapter 1}

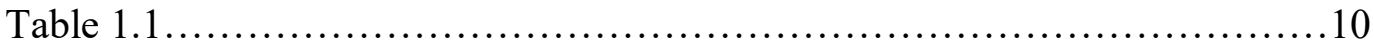

Summary of research on glucocorticoid levels in red-sided garter snakes

\section{Chapter 2}

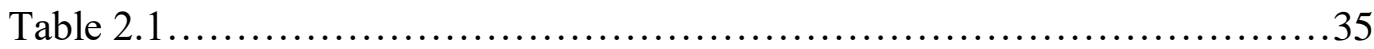
Influence of adrenocorticotropic hormone (ACTH) on plasma corticosterone in male and female red-sided garter snakes during the spring and fall.

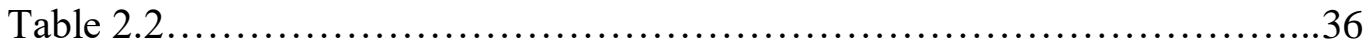
Influence of adrenocorticotropic hormone (ACTH) on plasma androgens in male red-sided garter snakes during the spring and fall. 


\section{Chapter 1}

\section{List of Figures}

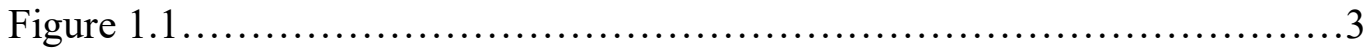

The hypothalamus-pituitary-adrenal (HPA) axis and associated hormones, corticotropin releasing hormone $(\mathrm{CRH})$, adrenocorticotropic hormone (ACTH), and glucocorticoids (GC).

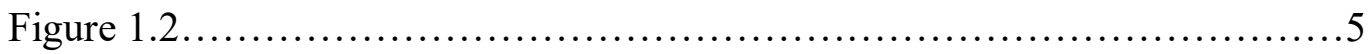

Red-sided garter snake life history cycle.

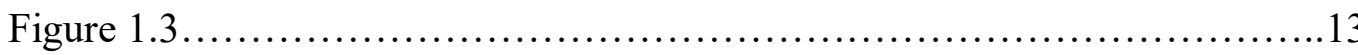

Capture stress methodologies.

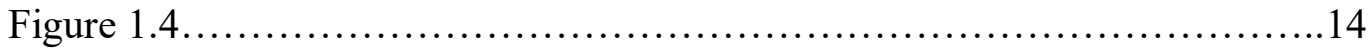

Capture stress can illustrate responsiveness of the HPA axis.

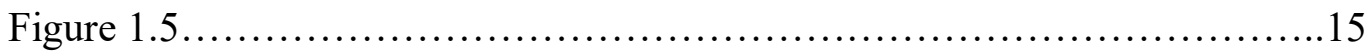

Negative feedback on the HPA axis.

\section{Chapter 2}

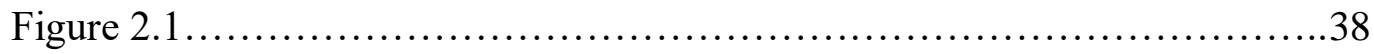

Effect of adrenocorticotropic hormone (ACTH) challenge on plasma corticosterone (mean $\pm \mathrm{SE}$ ) in male and female red-sided garter snakes (Thamnophis sirtalis parietalis) during the spring mating season (A and C, respectively) and fall pre-dormancy period ( $\mathrm{B}$ and $\mathrm{D}$, respectively).

Figure 2.2. .40

Influence of ACTH-induced changes in corticosterone on plasma androgens (mean $\pm \mathrm{SE}$ ) in male red-sided garter snakes (Thamnophis sirtalis parietalis) during the spring (A) and fall (B).

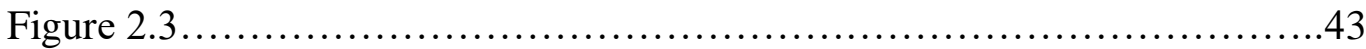
Mean integrated corticosterone response ( $+1 \mathrm{SE})$ to adrenocorticotropic hormone $(\mathrm{ACTH})$ challenge in male and female red-sided garter snakes (Thamnophis sirtalis parietalis) during the spring and fall (A).

\section{Chapter 3}

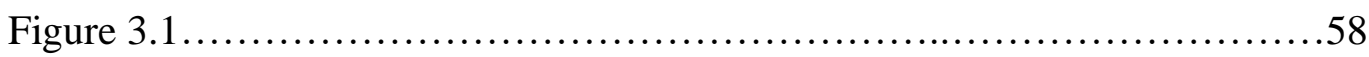

Calculation of the integrated corticosterone response. 
Figure 3.2..... 60

Corticosterone responses to capture stress $( \pm S E M)$ in female red-sided garter snakes (Thamnophis sirtalis parietalis) collected from the den and road during the spring mating season (A,B, respectively) and fall pre-dormancy period (C,D, respectively).

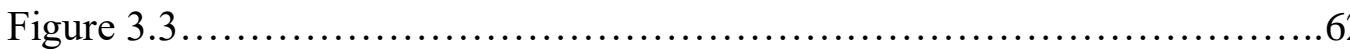

Baseline $(0 \mathrm{~h})$ corticosterone concentrations (A) and maximal corticosterone responses to capture stress (B) in female red-sided garter snakes (Thamnophis sirtalis parietalis) collected from the den and road during the spring and fall.

Figure 3.4 .

Integrated corticosterone responses to capture stress $( \pm$ SEM $)$ in female red-sided garter snakes (Thamnophis sirtalis parietalis) collected from the den and road during the spring and fall.

\section{Chapter 4}

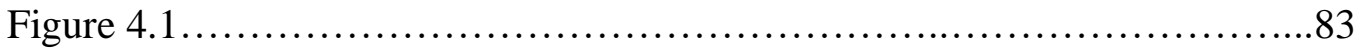

Effect of capture stress on mean plasma corticosterone $(+\mathrm{sem})$ of female redsided garter snakes ( $T$. sirtalis parietalis) before $(0 \mathrm{~h})$, during $(2 \mathrm{~h})$ and after $(4 \mathrm{~h})$ capture-stress treatment.

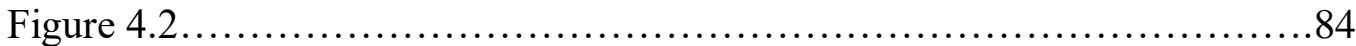

Regression of plasma corticosterone concentrations on body condition before ( 0 h) and during ( $2 \mathrm{~h}$ ) capture stress treatment in female red-sided garter snakes, $T$. sirtalis parietalis $(\mathrm{n}=23)$.

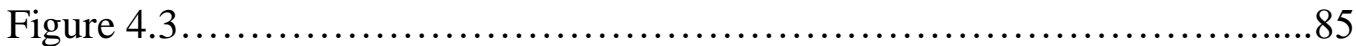

Influence of capture stress on mean latency to copulate (+ sem) in female redsided garter snakes ( $T$. sirtalis parietalis) with positive and negative body condition.

Figure $4.4 \ldots \ldots \ldots \ldots \ldots \ldots \ldots \ldots \ldots \ldots \ldots \ldots \ldots \ldots \ldots \ldots \ldots \ldots \ldots \ldots \ldots \ldots \ldots \ldots . . .68$

Effects of vehicle or exogenous corticosterone (CORT; low, $15 \mu \mathrm{g}$; high, $60 \mu \mathrm{g}$ ) on latency to copulate $(+\mathrm{sem})$ in female red-sided garter snakes $(T$. sirtalis parietalis) with positive and negative body condition.

\section{Chapter 5}

Figure 5.1.

Determination of glucocorticoid receptor (GR) in the brain of red-sided garter snakes (Thamnophis sirtalis parietalis) via Western blot. 


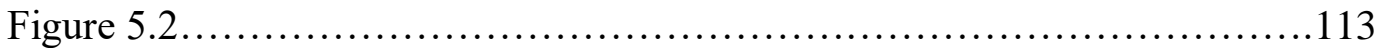

Corticosterone responses to capture stress in female red-sided garter snakes (Thamnophis sirtalis parietalis) in negative $(\mathrm{n}=35)$ and positive $(\mathrm{n}=28)$ body condition; receptive and unreceptive female groups were collapsed for analysis.

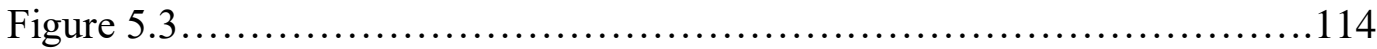

Corticosterone responses to capture stress in receptive $(\mathrm{n}=30)$ and unreceptive $(\mathrm{n}$ = 33) female red-sided garter snakes (Thamnophis sirtalis parietalis).

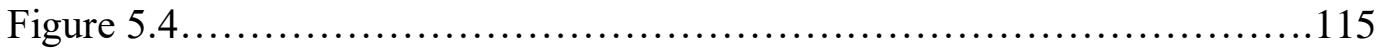

Relationship between reproductive condition and (A) body condition index at spring emergence, (B) stress-induced corticosterone concentrations, and (C) latency to copulate, a measure of proceptivity, in female red-sided garter snakes (Thamnophis sirtalis parietalis). 


\section{Preface}

Chapter 2 is published in the Journal of Experimental Biology

Dayger, C.A., Lutterschmidt, D.I., 2016. Seasonal and sex differences in responsiveness to adrenocorticotropic hormone contribute to stress response plasticity in red-sided garter snakes (Thamnophis sirtalis parietalis). J. Exp. Biol. 219 (7), 1022-1030.

Chapter 3 is published in General and Comparative Endocrinology

Dayger, C.A., Lutterschmidt, D.I., 2017. Patterns of stress responses shift during seasonal life-history transitions: an analysis comparing baseline, maximal and integrated corticosterone in female red-sided garter snakes (Thamnophis sirtalis parietalis). Gen. Comp. Endocrinol. 246, 29-36 .

Chapter 4 is published in Hormones and Behavior

Dayger, C.A., Cease, A.J., Lutterschmidt, D.I., 2013. Responses to capture stress and exogenous corticosterone vary with body condition in female redsided garter snakes (Thamnophis sirtalis parietalis). Horm. Behav. 64 (4), 748-754.

Chapter $\mathbf{5}$ is under review for Hormones and Behavior

Dayger, C.A., LeMaster, M.P., Lutterschmidt, D.I., Physiological correlates of reproductive decisions: Relationships among body condition, reproductive status, and the hypothalamus-pituitary-adrenal axis in a reptile. 


\section{Chapter 1}

\section{Introduction}

The availability of resources can fluctuate seasonally according to a cycle in environmental conditions. Consequently, seasonal animals modulate their physiology and behavior to align with the prevailing environmental conditions by integrating cues about their environment and internal state. By "seasonal" I mean that the animal experiences cyclic life-history stages that are appropriate for the changing environmental conditions (Jacobs, 1996). Jacobs (1996) defined “phenotypic stages," (life-history stages) which "represent major events in the life cycle, such as development, breeding, migration, and molting." Some of the cues that are reported to help time seasonal life-history stages, either as primary or supplementary cues, include day length, temperature, food availability, and social stimuli (Paul et al., 2008; Tamarkin et al., 1985). This process of integrating and responding to cues has high stakes, because animals risk decreased survival and fitness if they fail to appropriately time physiology and behavior. In the face of climate change, however, some cues no longer accurately predict environmental conditions. For example, in the arctic, female caribou migrate to their calving grounds so that they can give birth when there are rich and nutritious grazing opportunities (Post and Forchhammer, 2008). The cue they use to begin their journey is increasing day length, which is a consistent signal of the time of year. However, seasonal growth of the plants that caribou eat depend primarily on increasing temperatures, and thus, the peak food 
availability at the calving grounds is occurring earlier in the season relative to historical data due to climate change. By the time female caribou arrive at the calving grounds there is less forage available and it is less nutritious. Offspring mortality has increased as a result of this trophic mismatch (Post et al., 2001; Post and Forchhammer, 2008). It is critical to understand in detail the mechanisms animals use to transduce environmental cues into appropriate physiology and behavior for each life-history stage. Only with this information can we predict the impacts of global environmental change and manage vulnerable populations effectively.

One of the systems animals use to integrate external and internal cues is the hypothalamus-pituitary-adrenal (HPA) axis. The HPA axis has wide ranging effects on physiology and behavior and, in the context of a glucocorticoid stress response, is known to mediate tradeoffs between immediate survival and future fitness. An unexpected disturbance in an animal's environment can trigger a physiological stress response, causing a cascade of hormones to be released from the HPA axis. Environmental perturbations, deemed "stressors," trigger the release of corticotropin-releasing hormone (CRH) from the hypothalamus, which in turn stimulates the release of adrenocorticotropic hormone (ACTH) from the pituitary gland. ACTH causes the release of glucocorticoids from the adrenal glands (Fig. 1.1). Stress-induced changes in glucocorticoid hormones can coordinate a suite of physiological and behavioral changes, such as mobilizing energy stores, suppressing processes that are not essential to immediate survival, and modifying the immune system to facilitate immediate survival (Sapolsky, 2002; Wingfield et al., 1998). 
Beyond this stress response framework, glucocorticoids also play an important role in facilitating predictable life-history events (Landys et al., 2006). In this case, it is baseline glucocorticoids that play the primary role in mediating the changes in physiology and behavior during predictable life-history events. For instance, baseline glucocorticoid levels rise and fall in a 24-h diel rhythm, with relatively elevated levels occurring at the onset of the active phase. Baseline glucocorticoid concentrations also vary seasonally in many taxa, with elevated levels coinciding with migration in birds and fish and with the breeding season in birds, reptiles, amphibians, and some mammals (Iwata, 1995; Romero, 2002). Therefore, glucocorticoids may help animals coordinate the physiology and behavior that occurs during different life-history stages.

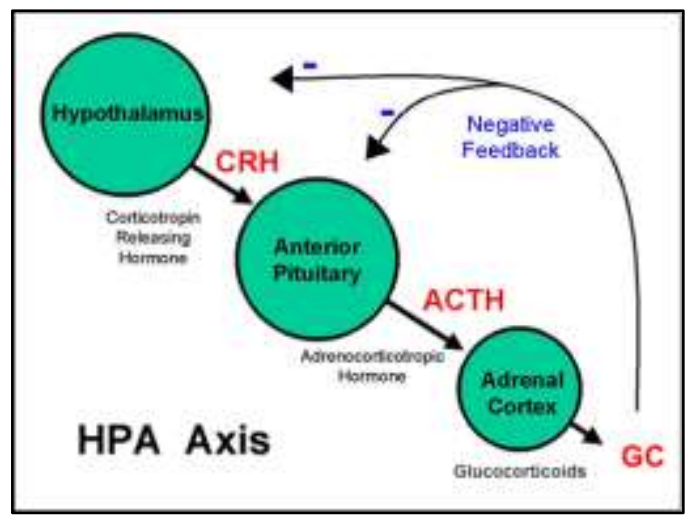

Figure 1.1. The hypothalamus-pituitaryadrenal (HPA) axis and associated hormones, corticotropin releasing hormone (CRH), adrenocorticotropic hormone (ACTH), and glucocorticoids (GC).

Glucocorticoid concentrations are often elevated during the mating season in a variety of taxa, presumably to fuel energetically demanding mating behavior (Landys et al., 2006). In at least some cases, elevated glucocorticoids are required for successful and/or sustained reproductive activity (Lutterschmidt and Maine, 2014; Schiml and 
Rissman, 1999). If glucocorticoid concentrations rise above a threshold, however, such as when exogenous glucocorticoids are administered, mating behavior can be suppressed (Dayger et al., 2013; Lutterschmidt et al., 2004; Moore and Miller, 1984; Moore and Mason, 2001; Salvante and Williams, 2003). For example, injection of exogenous glucocorticoids decreased sexual behavior in male rough-skinned newts in a dosedependent manner (Moore and Miller, 1984). Taken together, these data suggest that there is a range of optimal glucocorticoid concentrations, below which insufficient glucocorticoid concentrations cannot support reproduction. When glucocorticoid concentrations rise above the optimal range, they mediate a trade-off away from reproduction and to some other survival-promoting physiology and behavior.

Consequently, plasticity in HPA axis activity may have evolved to protect activities such as mating behavior or migration when baseline glucocorticoids are already high (Wingfield and Sapolsky, 2003). During periods when baseline glucocorticoids are elevated, stress may not induce an additional increase in glucocorticoids. This plasticity in stress responses likely maximizes reproductive opportunities. For example, animals with limited breeding opportunities often uncouple elevated glucocorticoids from suppression of mating behavior, allowing them to maximize reproductive opportunities even when faced with stressors (Moore et al., 2000a; Vitousek et al., 2010). Thus, there are times when the survival-promoting benefits of glucocorticoids are outweighed by the fitness-promoting benefits of suppressing the stress response.

Variation in HPA axis activity has been reported in all vertebrates, often in ways that contribute to coordinating seasonal reproduction and the transitions between life- 
history stages. The physiological mechanisms that produce this plasticity, however, are poorly understood. I aim to understand the role of the HPA axis, and specifically glucocorticoids, in seasonal reproduction in my model system. Relatively more studies have studied the role of the HPA axis in coordinating seasonal reproduction in males, and therefore much less is known about the role of glucocorticoids in seasonal reproduction in females. My dissertation research focuses on systematically examining the role of glucocorticoids in regulating seasonal life-history transitions, especially in females.

\section{Model organism}

I used red-sided garter snakes (Thamnophis sirtalis parietalis) as a model to investigate the role of the HPA axis in seasonal reproduction. Like all temperate animals, red-sided garter snakes have a distinct seasonal cycle of physiology and behavior (Fig. 1.2). During the freezing Manitoba winters, red-sided garter snakes aggregate underground in limestone dens below the frost line for up to 8 months. In the spring, roughly from mid-April to late-May, snakes emerge from the underground dens to

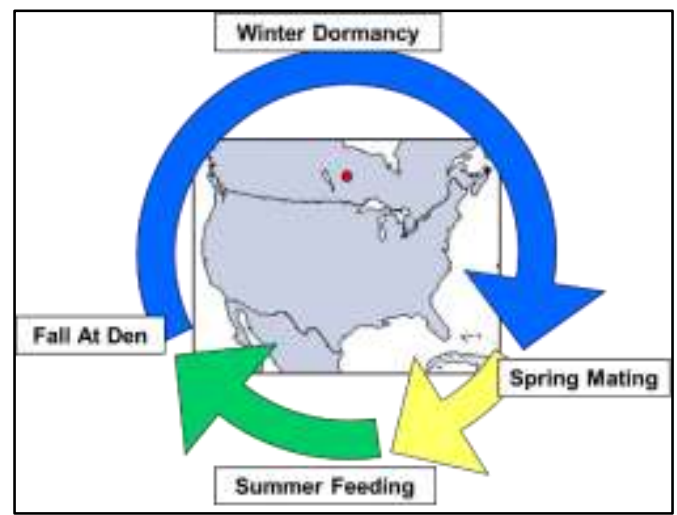

Figure 1.2. Red-sided garter snake life history cycle (modified from Krohmer and Lutterschmidt, 2011). 
engage in vigorous mating behavior. Males emerge first and remain congregated at the den site, awaiting emergence of females. Later in the mating season, females emerge and almost immediately dozens of males seek out and court each female, forming a mating ball often containing more than 50 snakes (Gregory, 1974; Gregory and Stewart, 1975; Shine et al., 2001). The short breeding season in which males and females must mate necessitates that reproductive behavior is remarkably robust to stressors. For instance, males will continue to seek, court and mate with females in the presence of researchers, and will even follow a female's pheromone trail to a researcher that has been handling females (Dayger, pers. observation).

After emergence, the female migrates away from the den site to the summer feeding grounds within about 24 hours (Gregory, 1974; Shine et al., 2001). Males remain at the den site to seek further mating opportunities, often for several weeks. Neither sex feeds during the spring mating season (Crews et al., 1987; Gregory and Stewart, 1975), and thus the energetically-demanding courtship behavior that males engage in can cause males to lose up to $1 \%$ of their body mass each day during the spring mating season (Shine et al., 2001). Like most reptiles, female garter snakes can store sperm (Birkhead and Møller, 1998; Sever and Hamlett, 2001). Females ovulate in the early summer and give birth to live young in the late summer (Gregory, 1977; Whittier and Crews, 1990). Females of live-bearing species in particular are subject to extraordinary energetic demands during reproduction, and some species cannot recover sufficient energy stores to reproduce in consecutive breeding seasons. Thus, female red-sided garter snakes are biennial breeders that give birth approximately every other year (Garstka et al., 1982; 
Gregory, 1977). A female's recent reproductive history can therefore influence whether or not she will reproduce in a given year. Males undergo spermatogenesis in the summer, storing their sperm over the winter for use the following spring. At the end of the summer, both males and females migrate back to the den site where they congregate for the pre-hibernation period before descending underground for the winter.

Red-sided garter snakes are capital breeders that rely on stored energy to fuel reproduction (Bonnet et al., 1998; Drent and Daan, 1980). Researchers often describe energy stores in ectotherms as the deviation from the relationship between body mass and body length, generally referred to as body condition. If an animal is heavier than expected for its length, it is said to be in high body condition with higher-than-average internal energy stores. In a given population, approximately $50 \%$ of females will have given birth more recently and have lower body condition. Females in higher body condition at emergence are significantly more likely to become vitellogenic, so body condition can be used as a proxy for recent reproductive history (Whittier and Crews, 1990). In males, body condition may influence how long it can engage in courtship behavior because males that were heavier-bodied at emergence stayed at the den site longer on average compared to lighter-bodied males (Shine and Mason, 2005).

As temporally dissociated breeders, male red-sided garter snakes do not rely on high sex steroid hormones during the breeding season to activate or sustain reproductive behavior (Crews, 1984; Krohmer et al., 1987; Lutterschmidt, 2012). In this species, cold temperature exposure is necessary to activate reproductive behavior (Garstka et al., 1982) and glucocorticoids are necessary to sustain that behavior once it has been initiated, at 
least in male snakes (Lutterschmidt and Maine, 2014). Therefore, the role of the HPA axis in seasonal reproduction can be studied without the confounding influence of sex steroids in red-sided garter snakes.

\section{Glucocorticoids in red-sided garter snakes}

Garter snakes are an exceptional model for studying the role of the HPA axis in seasonal reproduction because the reproductive behavior and endocrinology of this species has been more thoroughly investigated than in any other reptile, providing an invaluable framework for continued research (Krohmer and Lutterschmidt, 2011). Baseline glucocorticoid concentrations are generally elevated in males during the spring. Over the course of the spring mating season, baseline glucocorticoid concentrations gradually decline (Cease et al., 2007; Krohmer et al., 1987; Lutterschmidt and Maine, 2014) such that baseline concentrations are significantly lower in migrating males collected along their migratory route compared to courting males collected from the den site. Baseline glucocorticoids remain low during the summer in males and then rise again during the fall pre-hibernation period (Lutterschmidt and Mason, 2005; Moore et al., 2001; Whittier et al., 1987). During winter dormancy, there is a trend for baseline glucocorticoids to increase over time, but this effect was not statistically significant (Lutterschmidt, 2015).

In females, baseline glucocorticoids are generally elevated at spring emergence, decline to low levels over the summer, and increase again before the fall pre-dormancy period at the den site (Whittier et al., 1987). Whittier et al. (1987) reported that 
glucocorticoid concentrations of gravid female red-sided garter snakes did not differ from those of non-gravid females. However, baseline glucocorticoid concentrations increase at the end of gestation or immediately preceding parturition in other snake species (Schuett et al., 2004; Smith et al., 2012; Taylor et al., 2004). As opposed to the case in males, during winter dormancy, glucocorticoids increase significantly over time in females (Lutterschmidt, 2015).

The magnitude of stress-induced glucocorticoid levels can also vary seasonally in red-sided garter snakes. During the spring mating season, when baseline glucocorticoids are elevated, capture stress can produce a significant increase in glucocorticoids over baseline in male red-sided garter snakes in some cases (Lutterschmidt and Mason, 2010, 2005; Moore et al., 2000a; but see Cease et al., 2007; Moore et al., 2001). During the summer, when baseline glucocorticoid levels are low, capture stress elicits a significant increase in glucocorticoids in male red-sided garter snakes (Moore et al., 2001). During the fall, capture stress does not elicit a significant stress response in males (Lutterschmidt and Mason, 2005; Moore et al., 2001). Female stress responses have not yet been studied during any season, and thus it is unknown whether stress responses vary seasonally as they do in males, or if sex differences in the glucocorticoid stress response exist. A summary of research on baseline and stress-induced glucocorticoids in red-sided garter snakes is shown in Table 1.1.

\section{Methods to understand the function of the HPA axis}

The primary way to gain an understanding of HPA axis function is to challenge 
the axis in a variety of ways. One common strategy that has been used to assess the activity and sensitivity of the HPA axis is to subject an animal to a standardized stressor and then measure a variety of outcomes, including hormone concentrations, behavior, and immune function, among others. The sensitivity of the HPA axis can also be assessed by injecting exogenous hormones such as $\mathrm{CRH}$, ACTH or glucocorticoids and assessing the

\begin{tabular}{|c|c|c|c|c|c|}
\hline \multicolumn{2}{|c|}{$\begin{array}{c}\text { Season } \\
\text { (life history } \\
\text { stage) }\end{array}$} & \multirow{2}{*}{$\begin{array}{c}\begin{array}{c}\text { Spring } \\
\text { (mating) }\end{array} \\
\begin{array}{c}\text { High, decreases } \\
\text { over time }\end{array}\end{array}$} & \multirow{2}{*}{$\begin{array}{c}\begin{array}{c}\text { Summer } \\
\text { (feeding, } \\
\text { gametogenesis, } \\
\text { gestation) }\end{array} \\
\text { Low }\end{array}$} & \multirow{2}{*}{$\begin{array}{c}\text { Fall } \\
\text { (pre-hibernation) }\end{array}$} & \multirow{2}{*}{$\begin{array}{c}\begin{array}{c}\text { Winter } \\
\text { (dormancy) }\end{array} \\
\begin{array}{c}\text { Increases over } \\
\text { time }\end{array}\end{array}$} \\
\hline \multirow{2}{*}{$\stackrel{\frac{0}{\pi}}{\Sigma}$} & Baseline & & & & \\
\hline & $\begin{array}{r}\text { Stress } \\
\text { induced }\end{array}$ & $\begin{array}{l}\text { Not significantly } \\
\text { higher than } \\
\text { baseline* }\end{array}$ & $\begin{array}{l}\text { Significantly higher } \\
\text { than baseline }\end{array}$ & $\begin{array}{c}\text { Not significantly } \\
\text { higher than baseline }\end{array}$ & Unknown \\
\hline \multirow{2}{*}{ 节 } & Baseline & High & Low & Medium - high & $\begin{array}{c}\text { Increases over } \\
\text { time }\end{array}$ \\
\hline & $\begin{array}{r}\text { Stress } \\
\text { induced }\end{array}$ & Unknown & Unknown & Unknown & Unknown \\
\hline
\end{tabular}

resulting glucocorticoid concentrations or change in behavior. Each of the hormones that are secreted by the HPA axis bind to their corresponding receptors at other levels of the axis or in the periphery, and thus variation in the distribution or quantity of these receptors and which isoforms are expressed (Whitnall, 1993) can be assessed. Glucocorticoids negatively feed back on the HPA axis, controlling their own release via the strength of negative feedback (Whitnall, 1993). By eliciting a negative feedback response and measuring the rate of decline in endogenous glucocorticoids, variation in the strength of negative feedback can be examined. Finally, corticosteroid binding globulins (CBGs) circulate in the blood and bind glucocorticoids; they can either increase 
or decrease the impact of circulating glucocorticoids (Breuner et al., 2013; Breuner and Orchinik, 2002). All of these strategies have been employed by researchers in an effort to understand the mechanisms behind variation in HPA axis activity.

Capture and Restraint Stress. One of the most extensively used methods to probe the HPA axis is by investigating behavioral and hormonal responses to restraint or capture stress. Depending on the taxon being studied, restraint methods can vary from 15 minutes in amphibians (Moore and Miller, 1984), 30 minutes for birds and rodents (Romero et al., 2008, 1998), 90 minutes for fish (Ghisleni et al., 2012) and one to several hours for lizards and snakes (Dunlap and Wingfield, 1995; Moore et al., 2000a). Capture stress techniques are valuable because they stimulate the HPA axis in a physiological context with a standardized stressor. For example, Walker et al. (2015) used a standardized capture stress protocol to increase circulating glucocorticoid levels in free living penguins in different age classes (chick, juvenile, adult) and in different species (Spheniscus magellanicus, Spheniscus mendiculus, Eudyptes chrysocome). The protocol involved capturing a penguin, collecting a baseline blood sample within 3 minutes, covering its head with an opaque cloth bag and holding the restrained bird for 30 minutes. Another blood sample was collected at the end of the capture stress period before the birds were released. By comparing baseline and stress-induced glucocorticoid levels, Walker et al. (2015) discovered that juvenile penguins had a smaller stress response than adult penguins and Rockhopper penguins had a higher stress response than the other two species tested. The magnitude and duration of the stress-induced glucocorticoid increase can vary dramatically depending on a number of factors, including season, sex, internal 
energy stores, life-history stage, and reproductive history (Astheimer et al., 1995; Heath and Dufty, 1998; Kitaysky et al., 1999; Schoech et al., 1997; Zerani et al., 1991). For example, the stress-induced increase in glucocorticoids was smaller in molting male than non-molting male Lapland longspurs (Calcarius lapponicus; Astheimer et al., 1995). This avian example illustrates that capture stress methodologies can shed light on how HPA axis activity changes to accommodate different life-history events.

There are two main strategies for assessing capture stress-induced increases in glucocorticoids. The first is to sample a control group of animals and a separate group of capture stress-treated animals to compare animals undergoing two different experiences (e.g., Lutterschmidt and Mason, 2010). Blood samples from the non-stressed control group are generally paired during collection (Fig. 1.3A). The other method is to compare an individual animal's initial pre-stress sample to the post-stress sample (e.g., Moore et al., 2001) The within-individual method therefore corrects for individual variation in baseline glucocorticoid levels (Fig. 1.3B). Using either method, responses could be assessed at multiple sampling times over the course of the capture stress-treatment and therefore it is possible examine the responsiveness of the HPA axis. A more rapid increase in circulating glucocorticoids in one group would indicate that the HPA axis is more responsive in that group (Fig. 1.4).

HPA axis sensitivity. Another method of assessing the function of the HPA axis is to administer exogenous corticotropin releasing hormone $(\mathrm{CRH})$ or adrenocorticotropic hormone $(\mathrm{ACTH})$ and then measure the hormone response or other relevant output such as behavior or immune function. If the same dose of trophic hormone elicits different 
responses under different contexts, such as season, sex, or life-history stage, it reveals that HPA axis sensitivity to tropic hormones differs among those contexts (Cartledge and Jones, 2007; Girard et al., 1998; Phillips and Klukowski, 2008; Romero et al., 1998). For example, reproductively-active female skinks (Egernia whitii) increased glucocorticoids in response to exogenous $\mathrm{ACTH}$ administration more than females that were reproductively quiescent, suggesting that the adrenal glands are more responsive to

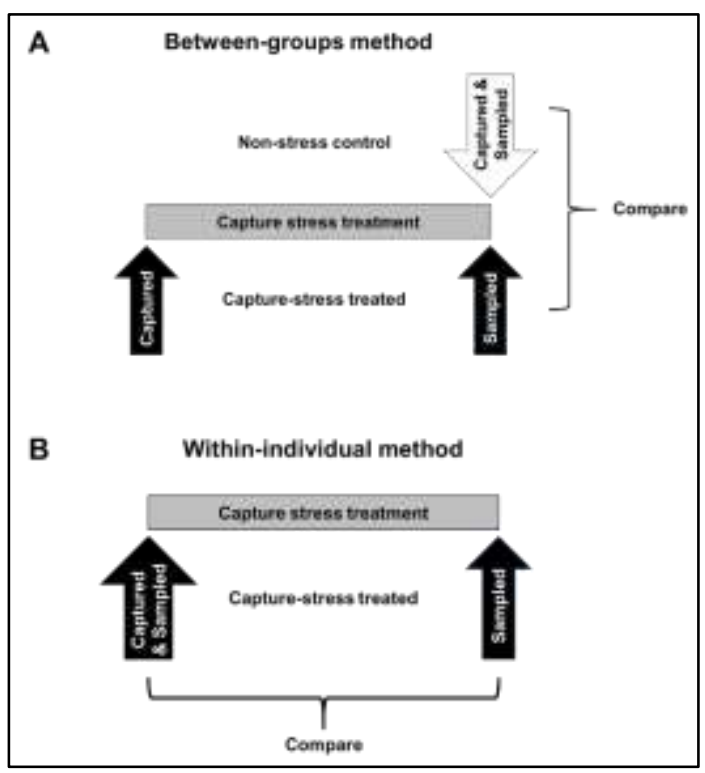

Figure 1.3. Capture stress methodologies. The between-groups method compares a capture stress-treated group to a non-stress control group (A). The within-individual method uses a repeated measures design to compare the post-stress sample to the pre-stress sample within each individual animal (B).

ACTH when females are reproducing than when they are non-reproductive (Cartledge and Jones, 2007). Furthermore, including a dose-response component to the experimental design can probe the sensitivity of the HPA axis in more detail. In Lapland longspurs treated with $100 \mathrm{IU}$ of ACTH during molt, the ACTH-treated birds increased glucocorticoids to a significantly higher level than vehicle-treated birds. During the 
breeding season, neither 100 IU nor 200 IU of ACTH elicited a significant increase in glucocorticoids, indicating that the adrenal glands of breeding birds are less sensitive to ACTH. The authors also assessed pituitary sensitivity to CRH by administering 1 of 2 doses of CRH and found that both adrenal sensitivity to ACTH and pituitary sensitivity to CRH were lower when longspurs were breeding compared to molting. These data suggest that the entire HPA axis was less sensitive during the breeding season. Administering tropic hormones to investigate sensitivity of the HPA axis can therefore uncover variation in the activity of the HPA axis beyond stress-induced reactivity.

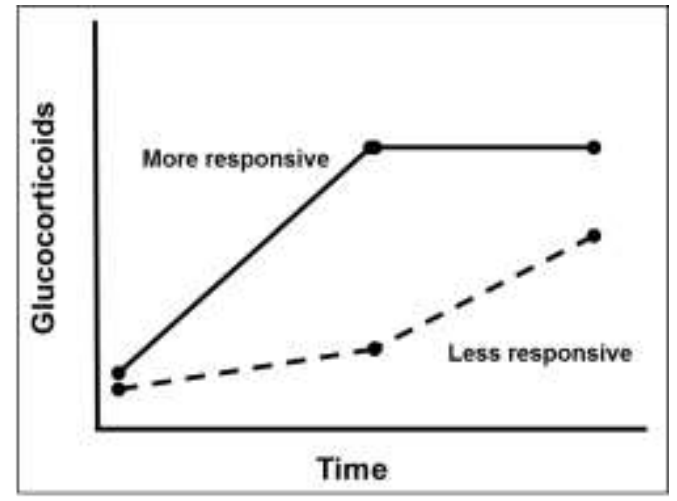

Figure 1.4. Capture stress can illustrate responsiveness of the HPA axis. A more rapid increase in glucocorticoids indicates that the HPA axis is more responsive (solid line). A more gradual increase indicates that the HPA axis is less responsive (dashed line).

Negative Feedback of the HPA axis. Glucocorticoid concentrations can also be modulated through negative feedback effects of elevated circulating glucocorticoids on the HPA axis at the level of the hypothalamus and pituitary gland. Researchers can use capture stress to raise circulating glucocorticoid levels and then administer a synthetic glucocorticoid such as dexamethasone to initiate negative feedback regulation of glucocorticoid production occurs. The advantage is that changes in endogenous 
glucocorticoids can be measured without measuring the synthetic glucocorticoid.

Negative feedback regulation occurs when plasma glucocorticoids bind to glucocorticoid receptors in CRH- and ACTH-producing cells. The strength of negative feedback, or how precisely glucocorticoid concentrations are maintained at homeostatic levels, can be modulated by changing the expression of receptors in the brain, primarily in the hippocampus and hypothalamus (Whitnall, 1993). Negative feedback is described as "strong" if elevated glucocorticoid levels decrease to initial levels quickly and "weak" if glucocorticoid levels remain elevated for a longer period of time (Fig. 1.5). For instance, in diurnal Galápagos marine iguanas (Amblyrhynchus cristatus), elevated, stress-induced glucocorticoid levels decrease rapidly following a dexamethasone injection administered during the day. At night, dexamethasone injection leads to only a modest decline in

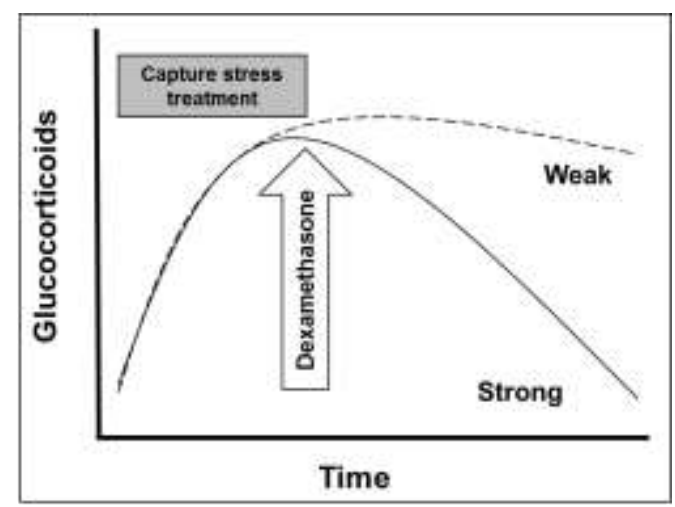

Figure 1.5. Negative feedback on the HPA axis. Capture stress treatment increases glucocorticoid concentrations and then dexamethasone, a synthetic glucocorticoid, is administered. When negative feedback on the HPA axis is "strong," glucocorticoid levels decrease rapidly; when "weak," they decrease gradually or not at all.

circulating glucocorticoid levels (Romero and Wikelski, 2006). Thus, negative feedback on the HPA axis is stronger during the day than at night. Previous studies in all vertebrate 
taxa demonstrate that the strength of negative feedback can vary with time of day, social rank, maternal care, time in captivity and brain region (Dickens et al., 2009; Liu et al., 1997; Mommsen et al., 1999; Nichols et al., 1965; Romero and Wikelski, 2006; Sapolsky, 1983; Yao et al., 2008). These data illustrate that variation in negative feedback may be a ubiquitous mechanism modulating plasma glucocorticoid concentrations.

Negative feedback regulation of plasma glucocorticoids is primarily accomplished by signaling at the hypothalamus and the hippocampus (Whitnall, 1993) via three receptors: a high affinity mineralocorticoid receptor (MR) responsible for baseline glucocorticoid signaling, a lower affinity glucocorticoid receptor (GR) primarily activated during stress-induced signaling, and a membrane-bound receptor (mGR) responsible for rapid, non-genomic effects (Landys et al., 2006; Whitnall, 1993). Because low-affinity GR is bound when circulating glucocorticoid concentrations are relatively high, the suppression of the stress response via negative feedback is likely carried out through signaling at the GR receptor (Whitnall, 1993). Therefore, variation in the amount of brain GR can impact the strength of negative feedback on the HPA axis. Researchers can compare GR mRNA and/or protein content in the brain to assess the strength of negative feedback on the HPA axis. For example, in mice separated from their dams for 180 minutes every day as pups, GR mRNA density in the brain and the strength of negative feedback regulation of the HPA axis was decreased (and therefore glucocorticoid concentrations remain high after stress; Ladd et al., 2004). GR is widely distributed in the brain, but the hypothalamus and hippocampus are common targets of 
negative feedback regulation (Whitnall, 1993). There is some evidence that signaling through MR (Ratka et al., 1989), and/or the ratio of brain MR:GR (Ladd et al., 2004) impacts the strength of negative feedback of the HPA axis, and thus assessing GR and MR content may provide a more complete understanding of negative feedback capacity than assessing GR alone.

Glucocorticoid Inhibitors. An alternative approach to investigating HPA axis activity is to decrease, rather than increase, circulating glucocorticoids. Three drugs have been used in the literature to inhibit either glucocorticoid synthesis or glucocorticoid receptor signaling. Metyrapone is a glucocorticoid synthesis inhibitor that reduces plasma glucocorticoid levels (i.e., Belda et al., 2012; Lutterschmidt and Maine, 2014; Schiml and Rissman, 1999; Yao et al., 2008), mitotane reduces circulating glucocorticoid levels, probably by modifying glucocorticoid clearance (e.g., Breuner and Orchinik, 2001; Terzolo et al., 2014), and mifepristone (also called RU486) antagonizes glucocorticoid receptor signaling (i.e., Belda et al., 2012; Katz et al., 2008; Weiss et al., 2007). While capture stress and tropic hormone administration both generally raise circulating glucocorticoid levels, metyrapone and mifepristone can decrease plasma glucocorticoid concentrations or signaling, respectively, which offers a distinct approach to uncovering the role of glucocorticoids in physiological processes. For example, Lutterschmidt and Maine (2014) demonstrated that experimentally decreasing glucocorticoid concentrations with implants containing metyrapone caused male red-sided garter snakes to prematurely transition to feeding behavior. These data suggest that elevated baseline glucocorticoids are necessary to sustain courtship behavior during the spring in male red-sided garter 
snakes. Experimentally increasing glucocorticoids by any means would not have uncovered this role for glucocorticoids in supporting courtship behavior. In addition, it has been reported that glucocorticoid-releasing implants do not consistently increase circulating glucocorticoids long-term because endogenous negative feedback on the HPA axis can compensate for the glucocorticoid increase (Robertson et al., 2015). Therefore, decreasing glucocorticoids can offer a different perspective on HPA axis regulation.

Each of the methods described investigates different characteristics of the HPA axis. Systematically applying a variety of methods to examine the HPA axis is a powerful approach to understanding how the entire HPA axis responds in different contexts. The literature indicates that HPA axis activity is highly context-dependent and variation in HPA axis activity likely plays a role in supporting seasonal life-history stages. In the following chapters, I have used many of these methods to characterize the role of the HPA axis in seasonal reproduction, especially in females.

\section{Rationale for the chapters}

Baseline glucocorticoid concentrations vary seasonally in male and female redsided garter snakes. Males are also less responsive to capture stress during the spring mating season compared to other times of year. One way that animals adjust baseline glucocorticoids and stress responses is via modulation of HPA axis sensitivity, which can also vary between seasons and life-history stages (e.g., Romero and Wingfield, 1998). Thus, reduced adrenal responsiveness could underlie reports of reduced stress sensitivity during the spring. In Chapter 2, I hypothesized that plasticity in stress responses is 
regulated, in part, by changes in the responsiveness of the adrenal glands to ACTH. To test this hypothesis, I compared hormonal responses to ACTH challenge during the spring and fall. Because female stress responses have not yet been investigated, it is unknown whether females show decreased stress responsiveness during the spring compared to other seasons as males do, or if there is a sex difference in HPA axis activity. Therefore, I compared responses to ACTH during the spring and the fall in both males and females to investigate potential season-by-sex variation.

Because female stress responses in red-sided garter snakes have not been investigated, it is unknown whether the seasonal differences in stress-induced glucocorticoid concentrations are specific to males or also occur in females. My findings in Chapter 2 led me to ask in Chapter 3 whether the lack of a seasonal difference in adrenal responsiveness to ACTH in females corresponded to consistent stress responses between seasons. I hypothesized that stress responses vary seasonally in females, as they do in males. To test this hypothesis, I compared female stress responses during the spring and fall to uncover potential seasonal variation. This experiment also allowed me to uncover potential sex differences in stress responses. Females migrate away from the den more quickly after emerging than males, indicating there is a sex difference in the duration of the mating season. Therefore, I also assessed the stress responses of females during migration to investigate if potential sex differences in HPA axis activity could explain sex differences in the timing of migration.

Female red-sided garter snakes are biennial breeders that give birth approximately every other year (Gregory, 1977; Garstka et al., 1982). A female's recent reproductive 
history can therefore influence whether or not she will reproduce in a given year. In at least some species, HPA axis sensitivity varies with female reproductive state (Cartledge and Jones, 2007; Lutterschmidt et al., 2009; Taylor et al.,2004; Vitousek et al., 2010), which suggests that HPA axis activity may vary with recent reproductive history in redsided garter snakes. Body condition can be used as a proxy for recent reproductive history (Whittier and Crews, 1990) and can be related to baseline and/or stress-induced glucocorticoid concentrations (e.g., Moore et al., 2001, 2000b; Vitousek et al., 2010), although not all studies report this relationship (e.g, Cease et al., 2007; Moore et al., 2000). In Chapter 4, I hypothesized that stress responses vary with body condition. To test this hypothesis, I compared glucocorticoid stress responses and female mating behavior in females in varying body conditions. I also compared female mating behavior in females treated with one of three doses of exogenous glucocorticoids to investigate if sensitivity to glucocorticoids varied with body condition.

In Chapter 4, I learned that female hormonal and behavioral stress responses vary with body condition. Because body condition is likely related to reproductive history, I directly tested in Chapter 5 if there is a relationship among body condition, reproductive history and HPA axis activity. Variation in female stress responses with body condition could be the result of variation in GR protein in the brain. Therefore, I investigated if GR varied with body condition in females. Chapters 2 and 3 both discussed sex differences in HPA axis activity during the spring. Based on these data, and because brain GR has not been examined in red-sided garter snakes in either sex, I also compared brain GR protein in males and females during the spring. 
Together, these chapters help characterize the role of glucocorticoids in seasonal reproduction in male and especially female red-sided garter snakes. My dissertation research investigates how HPA axis activity varies with season, sex, reproductive history and migration status. By systematically probing the HPA axis in a single, tractable system, I have gained insight into how changes in the HPA axis support and modulate transitions between life-history stages. These results highlight the HPA axis' important function in mediating critical trade-offs all animals must navigate to be successful. A better understanding of the mechanisms that dictate why animals do what they do, and when they do them, can improve our ability to predict how animals respond in the face of a rapidly changing world. 


\title{
Chapter 2
}

\section{Seasonal and sex differences in responsiveness to adrenocorticotropic hormone contribute to stress response plasticity in red-sided garter snakes}

(Thamnophis sirtalis parietalis)

Published in the Journal of Experimental Biology, 219(7), 1022-1030.

\begin{abstract}
Like many vertebrates, hormonal responses to stress vary seasonally in red-sided garter snakes (Thamnophis sirtalis parietalis). For example, males generally exhibit reduced glucocorticoid responses to a standard stressor during the spring mating season. We asked whether variation in adrenal sensitivity to adrenocorticotropic hormone (ACTH) explains why glucocorticoid responses to capture stress vary with sex, season, and body condition in red-sided garter snakes. We measured glucocorticoids at 0,1 , and 4 hours after injection with ACTH (0.1 IU/g body mass) or vehicle in males and females during the spring mating season and fall pre-hibernation period. Because elevated glucocorticoids can influence sex steroids, we also examined androgen and estradiol responses to ACTH. ACTH treatment increased glucocorticoids in both sexes and seasons. Spring-collected males had a smaller integrated glucocorticoid response to ACTH than fall-collected males. The integrated glucocorticoid response to ACTH differed with sex during the spring, with males having a smaller glucocorticoid response than females. Although integrated glucocorticoid responses to ACTH did not vary with
\end{abstract}


body condition, we observed an interaction among season, sex and body condition. In males, ACTH treatment did not alter androgens in either season, but androgens decreased during the sampling period. Similar to previous studies, plasma estradiol was low or undetectable during the spring and fall and therefore any effect of ACTH treatment on estradiol could not be determined. These data provide support for a mechanism that partly explains how the HPA axis integrates information about season, sex, and body condition: namely, variation in adrenal responsiveness to ACTH.

\section{Introduction}

In light of the increasing impacts of climate change, habitat loss and fragmentation, endocrine disruption and loss of biodiversity on communities, it is critical that we understand the neuroendocrine mechanisms animals use to integrate information about their environment with their internal state to produce appropriate physiology and behavior. A cohesive understanding of these mechanisms has yet to emerge. The hypothalamus-pituitary-adrenal (HPA) axis is likely central to these mechanisms, because this hormone system enables an animal to integrate multiple signals (i.e., environmental conditions, life-history stage, reproductive history or status, and internal energy balance) and then respond appropriately by modulating circulating hormone concentrations.

In response to an acute perturbation or "stressor," the HPA axis secretes glucocorticoid hormones, which coordinate changes in physiology and behavior that promote immediate survival by mobilizing energy stores and suppressing non-essential processes such as reproduction and digestion (Wingfield, 2008). Importantly, the 
magnitude of the stress-induced increase in glucocorticoids is often context dependent, varying with season (Astheimer et al., 1995; Klukowski, 2011; Lutterschmidt et al., 2009; Moore et al., 2001), sex (Cartledge and Jones, 2007; Pottinger et al., 2013), reproductive state (Cartledge and Jones, 2007; Lutterschmidt et al., 2009), and body condition (Dayger et al., 2013; Heath and Dufty, 1998). For example, male fence lizards (Sceloporus undulatus) generally respond to a standardized capture stress protocol by increasing plasma glucocorticoids. During the breeding season, however, the maximum glucocorticoid concentration attained is smaller compared to the nonbreeding season (Klukowski, 2011). Whether such variation in stress responses results from a change in the capacity of the adrenal glands to secrete glucocorticoids, upstream changes in the hypothalamus or pituitary, or a combination of both, is often unknown.

In addition to context-dependent variation in glucocorticoid synthesis, the responses to increased glucocorticoids, both physiological and behavioral, can also vary with context. For example, exogenous corticosterone treatment increases plasma glucocorticoid concentrations in both territorial orange-blue and non-territorial orange morphs of male tree lizards (Urosaurus ornatus). However, elevated corticosterone elicits a greater suppression of plasma testosterone in orange morphs compared to orange-blue morphs (Knapp and Moore, 1997). In another example, a low physiological dose of exogenous corticosterone suppresses mating behavior only in female red-sided garter snakes (Thamnophis sirtalis parietalis) in below-average body condition; mating behavior of females in above-average body condition is not significantly affected (Dayger et al., 2013). Thus, there are contexts in which elevated glucocorticoid 
concentrations suppress neither sex steroid hormones nor mating behavior, but instead may facilitate reproduction by fueling energetically costly mating behavior (Lutterschmidt and Maine, 2014; Wingfield and Sapolsky, 2003). Indeed, many vertebrates exhibit elevated baseline glucocorticoids during energetically challenging life-history stages such as reproduction (reviewed in Landys et al., 2006; Moore and Jessop, 2003).

While the mechanisms mediating context-dependent variation in stress-induced glucocorticoids are poorly understood, a change in the sensitivity of the HPA axis is likely involved (Romero, 2002; Romero and Wingfield, 2001; Wingfield et al., 1994). One way to better understand the mechanisms underlying plasticity in stress responses is to administer an adrenocorticotropic hormone (ACTH) “challenge.” Administering a sufficiently high dose of pituitary ACTH induces the adrenal glands to secrete maximal levels of glucocorticoids. Comparing maximal glucocorticoid concentrations among contexts (e.g., life history stages, reproductive stages) can reveal how the sensitivity of the adrenal glands varies with those contexts. For example, snow buntings (Plectrophenax nivalis) respond to restraint stress with a larger glucocorticoid response during breeding compared to molting. The higher sensitivity to restraint stress during the breeding season is concomitant with increased sensitivity of the adrenal glands to ACTH (Romero et al., 1998). These and other studies demonstrate that variation in HPA axis sensitivity may mediate the effects of context on stress-induced changes in glucocorticoids and, by extension, physiology and behavior (reviewed in Wingfield, 2008). 
We examined whether variation in responsiveness to ACTH could explain observed differences in hormonal responses to capture stress in a well-studied population of red-sided garter snakes (Thamnophis sirtalis parietalis) in Manitoba, Canada. Following spring emergence from winter dormancy, red-sided garter snakes exhibit mating behavior for approximately 4 weeks. Female snakes generally leave the den postcopulation, but males remain near the den for several weeks, searching for mates and courting emerging females (Shine et al., 2001). Once snakes leave the den, they migrate up to $18 \mathrm{~km}$ to summer feeding areas (Gregory and Stewart, 1975). Neither sex feeds during winter dormancy or while at the den site, but because females migrate away within a few days, they likely have the opportunity to forage more quickly after emergence (Crews et al., 1987; Shine et al., 2001). In the fall, snakes migrate back to the den site before returning underground for winter dormancy.

Baseline glucocorticoids are generally elevated during the spring mating season compared to other times of year, likely to support the energetic demands of mating behavior (Lutterschmidt and Maine, 2014; Moore et al., 2001; Whittier et al., 1987). Redsided garter snakes also exhibit a temporally-dissociated reproductive pattern, whereby peak gonadal activity is temporally dissociated from mating behavior (Crews, 1984). In other words, mating behavior of red-sided garter snakes during the spring occurs when sex steroids tend to be low (reviewed in Lutterschmidt, 2012). Androgens in males and estrogens in females then rise during the summer when males commence spermatogenesis and females undergo vitellogenesis, ovulate and become gravid (Krohmer et al., 1987; Whittier et al., 1987). Estradiol rapidly declines after parturition in 
females (Whittier et al., 1987), but androgens in males remain significantly elevated in the fall compared to spring (Lutterschmidt and Mason, 2005; Lutterschmidt and Mason, 2009; Moore et al., 2001).

During the fall, capture stress elicits a significant increase in corticosterone (the primary glucocorticoid in reptiles) in male red-sided garter snakes, but not consistently during the spring (Lutterschmidt and Mason, 2005; Moore et al., 2000a; Moore et al., 2001). In addition, responses to capture stress vary with body condition. For example, male reptiles and amphibians in below-average body condition sometimes have higher baseline and stress-induced glucocorticoids (reviewed in Moore and Jessop, 2003). The relationship between glucocorticoids and body condition is important because the energetically demanding courtship and mating behavior in which male garter snakes engage can result in males losing an average of $10 \%$ of their body mass over the course of the mating season (O'Donnell et al., 2004). Thus, body condition may play a role in determining how long an individual male is capable of seeking reproductive opportunities (Shine and Mason, 2005). Similar to males, female responses to capture stress during the spring mating season also depend on body condition (Dayger et al., 2013). Females in below-average body condition significantly increased glucocorticoid concentrations in response to capture stress, while females in above-average body condition did not.

Taken together, these data suggest that the sensitivity of the HPA axis varies with season, sex and body condition in red-sided garter snakes. Here, we test the hypothesis that the observed variation in stress-induced glucocorticoid concentrations is related to the responsiveness of the adrenal glands to ACTH. Because elevated glucocorticoids, like 
those expected with ACTH challenge, are often associated with a decrease in sex steroid hormone concentrations (e.g., Duckworth et al., 2001; Hau et al., 2010; Leary and Harris, 2012; Leary et al., 2004; Moore et al., 1991; Moore et al., 2000a; Wingfield and Sapolsky, 2003), we also examined potential effects of ACTH-induced glucocorticoids on androgens in males and estradiol in females. We predicted that differences in the sensitivity of the adrenal glands to ACTH would mirror previously-reported variation in responses to capture stress between seasons, sexes, and body conditions. Principally, we asked if the sensitivity of male snakes to ACTH is reduced during the spring mating season and if responses to ACTH, particularly during the spring mating season, vary with sex and/or body condition.

\section{Materials and methods}

The experiment described here was conducted in the field with free-ranging redsided garter snakes (Thamnophis sirtalis parietalis) at a den site located in Inwood, Manitoba, Canada. Snakes were collected during the spring mating season from 15-18 May 2012 and before they descended underground for winter dormancy from 12-15 September 2012. This experiment was approved by the Manitoba Department of Conservation (scientific permit number WB14930) and Portland State University's Institutional Animal Care and Use Committee (approval number psu12.05.15.1).

\section{Experimental Design and ACTH treatment}

We collected 40 male and 43 female snakes during the spring and 39 males and 
42 females during the fall. Females were confirmed to be unmated before including them in the experiment by checking for the absence of a mating plug. A blood sample $(150 \mu \mathrm{L})$ was collected from the caudal vein immediately upon capture $(t=0 \mathrm{~h}$; mean sampling time \pm SE: $81 \mathrm{~s} \pm 4.8 \mathrm{~s}$ ). Snakes were then weighed and randomly assigned to either $\mathrm{ACTH}$ or vehicle treatment. For ACTH treatment, an intraperitoneal injection of $0.1 \mathrm{IU}$ ACTH/g body mass (product no. A6303, Sigma-Aldrich, St. Louis, MO, USA) was administered from a stock solution containing $20 \mathrm{IU} / \mathrm{mL}$ reptilian Ringer's solution. Injection volume was $0.5 \%$ of body mass. We chose this $\mathrm{ACTH}$ dose because it elicits a large increase in plasma glucocorticoids in lizards and birds (Klukowski, 2011; Phillips and Klukowski, 2008; Romero and Wingfield, 1999). Vehicle-treated snakes received an equivalent volume of reptilian Ringer's solution.

Following treatment, snakes were marked with a unique color pattern on the dorsal stripe using colored marker to identify individuals. Males and females were transferred to separate arenas $(1 \mathrm{~m} \times 1 \mathrm{~m} \times 1 \mathrm{~m})$ with hiding areas for the duration of the experiment; housing snakes in these arenas does not elicit a significant change in glucocorticoids (Moore and Mason, 2001). Less than 7 minutes elapsed from initial collection of the snake to its transfer to the holding arena. Additional blood samples were collected from each snake 1 hour and 4 hours after injection $(150 \mu \mathrm{L}$ at each sampling time). At the conclusion of the experiment, snout-vent length was measured for each snake. Snakes were then released at the site of capture within 24 hours of collection; the colored marker patterns on the dorsal stripe enabled us to avoid recapturing a previously sampled snake. To calculate body condition, we regressed body mass on snout-vent 
length to enable comparisons to previous studies (Cease et al., 2007; Dayger et al., 2013; Jessop et al., 2004; Moore et al., 2000b). We designated snakes with positive residuals as being in positive body condition and vice versa. Final sample sizes within each treatment group were 21 male and 22 female snakes treated with ACTH during the spring, 19 male and 21 female snakes treated with vehicle during the spring, 20 male and 21 female snakes treated with ACTH during the fall, and 19 male and 21 female snakes treated with vehicle during the fall. Final sample sizes within body condition category for each analysis are listed in the figures.

\section{Blood sampling and radioimmunoassay}

Blood samples were collected using heparinized 25-g needles and 1-cc syringes. Blood samples were stored on ice before returning to the field station, where they were centrifuged to separate plasma from blood cells. The plasma was transferred to fresh tubes and stored at $-4^{\circ} \mathrm{C}$, transported on dry ice to Portland State University and stored at $-80^{\circ} \mathrm{C}$ until analysis. Plasma hormone concentrations (corticosterone and estradiol for females; corticosterone and androgens for males) were determined for each sample by direct radioimmunoassay using the methods described and validated by Dayger et al. (2013) and Lutterschmidt and Mason (2005; 2009). Briefly, steroid hormones were extracted from 5-70 $\mu \mathrm{L}$ of plasma with anhydrous ethyl ether, and then evaporated under nitrogen gas in a $37^{\circ} \mathrm{C}$ bead bath. Hormone extracts were reconstituted in phosphate buffered saline. Samples were assayed in duplicate for each hormone, and each replicate aliquot was incubated with approximately $12,000 \mathrm{cpm}\left[1,2,6,7-{ }^{3} \mathrm{H}\right]-$ corticosterone, 
$\left[2,4,6,7-{ }^{3} \mathrm{H}\right]$ - estradiol, or $\left[1,2,6,7-{ }^{3} \mathrm{H}\right]$ - testosterone (Product numbers: NET399250UC, NET317250UC, NET370250UC, respectively; Perkin-Elmer, Piscataway, NJ, USA) and $100 \mu \mathrm{L}$ antiserum at $4^{\circ} \mathrm{C}$ for $18-24$ hours (corticosterone antibody: 07120016 from MP Biomedicals, LLC, Solon, OH, USA; estradiol and testosterone antibody: 20R-ER012w and 20R-TR018w, respectively, from Fitzgerald Industries International, Acton, MA, USA). Unbound steroid was separated from bound steroid using dextran-coated charcoal and the radioactivity of the bound fraction was quantified using a liquid scintillation counter (Beckman Model No. 6500). The testosterone antibody we used significantly cross-reacts with 5- $\alpha$-dihydrotestosterone (63\% cross reactivity; Fitzgerald Industries International), and therefore we report data for androgens rather than testosterone concentrations.

Within each sex, samples were randomly distributed across assays. Samples were run in 8 assays for corticosterone, 4 assays for estradiol, and 6 assays for androgens, with mean intra-assay coefficients of variation of $11.1 \%$ for corticosterone, $11.8 \%$ for estradiol and $8.0 \%$ for androgens. The mean inter-assay coefficients of variation were $18.0 \%$ for corticosterone, $19.3 \%$ for estradiol, and $15.1 \%$ for androgens. Mean percent recovery was $78.2 \%$ for male samples and $71.4 \%$ for female samples; concentrations were corrected for individual recovery variation. The average limit of detection was $29.3 \mathrm{pg} / \mathrm{mL}$ for corticosterone, $7.4 \mathrm{pg} / \mathrm{mL}$ for estradiol and $12.9 \mathrm{pg} / \mathrm{mL}$ for androgens.

\section{Statistical analyses}

We used SigmaPlot 12.0 software to perform all statistical analyses (Systat 
Software 2010, Systat systems, Inc., Point Richmond, CA, USA). All statistical comparisons were considered significant at $P \leq 0.05$. All data met the assumptions for parametric analysis (i.e., normality and equal variance) unless otherwise noted. In 3 of the 6 repeated measures analyses that we performed (i.e., fall corticosterone data from males and females and spring androgen data from males), data transformation could not correct for non-normality. Because non-parametric options for multifactorial repeated measures analysis of variance (ANOVA) do not exist, we proceeded with parametric analyses similar to Lutterschmidt and Mason $(2008,2009)$ because ANOVAs are robust against modest deviations from normality (Sheskin, 2007; Zar, 1999). For these analyses, we chose a transformation that most closely approximated normality based on a ShapiroWilk normality test. Some male samples had undetectable concentrations of corticosterone ( $\mathrm{n}=1$ of 237 samples, $0.4 \%)$ and/or androgens $(\mathrm{n}=8$ of 237 samples, $3.4 \%$ ) and were assigned the limit of detectability for analysis. Estradiol concentrations in most female samples (227 of 255 samples, $89 \%$ ) were below the limits of detectability, so no statistical tests were performed on these data.

We first verified that ACTH treatment significantly increases plasma corticosterone concentrations over time using a two-way repeated measures ANOVA with treatment and sampling time as factors; each sex was analyzed separately within each season. We also examined if ACTH-induced changes in corticosterone alter androgen concentrations of male snakes using a two-way repeated measures ANOVA within each season with treatment and time as factors. We further investigated significant main effects of each ANOVA using a Student-Newman-Keuls multiple comparisons 
procedure.

To better understand the overall corticosterone response to ACTH challenge, we calculated an integrated corticosterone response for each ACTH-treated snake similar to the methods of Cockrem and Silverin (2002) and Rensel and Schoech (2011). For each snake, corticosterone concentration was plotted over time and the area under the curve was calculated using the trapezoid rule with Microsoft Excel (Microsoft Office 2010, Redmond, WA, USA). We corrected for variation in baseline corticosterone concentrations across individual snakes by multiplying the baseline corticosterone concentration by the entire 4 hour sampling period and subtracting this value from the total area under the curve. This procedure generated the corrected integrated corticosterone response (hereon referred to as the integrated corticosterone response). We then used these data to evaluate differences in the overall corticosterone response to ACTH treatment among snakes, as the integrated data incorporate, at least in part, temporal patterns in both the response to and recovery from ACTH treatment. Because transformation could not correct for deviations in normality, we performed a nonparametric three-way ANOVA using the Scheirer-Ray-Hare extension of a KruskallWallis ANOVA on the integrated corticosterone response; season, sex and body condition were included as factors. There was a significant three-way interaction in this analysis, and therefore we reanalyzed the integrated corticosterone response data to further examine the potential effects of season and sex. Based on a priori knowledge, we focused our analyses on seasonal differences within males and potential sex differences within the spring. Within males, we reanalyzed the integrated corticosterone data with a 
non-parametric two-way ANOVA with season and body condition as factors. We compared the integrated corticosterone response of males and females within the spring by reanalyzing the data using a non-parametric two-way ANOVA with sex and body condition as factors.

\section{Results}

\section{Effects of ACTH treatment on corticosterone}

We first verified that ACTH treatment elicits an increase in plasma corticosterone concentrations in each season and sex separately. The main effects of sampling time were statistically significant in all analyses (Table 1). The main effect of treatment was statistically significant only in spring-collected males, but we observed a significant interaction between treatment and sampling time in all other groups (i.e., fall-collected males and in females collected during both the spring and fall). As expected, these results indicate that the effect of ACTH treatment on corticosterone depends on sampling time.

Compared to vehicle treatment, ACTH treatment significantly increased plasma corticosterone in males during the spring and in both sexes during the fall at the $1 \mathrm{~h}$ sampling time (Fig. 2.1A-D; from Student-Newman-Keuls multiple comparisons procedures). In males collected during fall and in females collected during both spring and fall, ACTH-induced corticosterone concentrations remained elevated at the $4 \mathrm{~h}$ sampling time (Figs. 2.1B - D). In contrast, plasma corticosterone of ACTH-treated, spring-collected males declined to below-baseline levels at the $4 \mathrm{~h}$ sampling time. Vehicle-treated males collected during spring did not change plasma corticosterone 
significantly from baseline throughout the entire sampling period (Fig. 2.1A). During the fall, vehicle-treated males increased plasma corticosterone above baseline at the $1 \mathrm{~h}$ and 4 h sampling times, although not to ACTH-induced levels (Fig. 2.1B). Vehicle-treated females during both the spring and fall did not significantly increase plasma corticosterone above baseline levels at any sampling time (Figs. 2.1C, D).

\begin{tabular}{|c|c|c|c|c|}
\hline Season & Sex & Treatment & Time & Interaction \\
\hline Spring & $\sigma^{x}$ & $\begin{array}{l}\mathrm{F}_{1,119}=6.240 \\
P=\mathbf{0 . 0 1 7}\end{array}$ & $\begin{array}{l}\mathrm{F}_{2,119}=5.480 \\
P=\mathbf{0 . 0 0 6}\end{array}$ & $\begin{array}{l}\mathrm{F}_{2,119}=2.912 \\
P=0.060\end{array}$ \\
\hline Spring & $q$ & $\begin{array}{l}\mathrm{F}_{1,128}=0.536 \\
P=0.468\end{array}$ & $\begin{array}{l}\mathrm{F}_{2,128}=10.756 \\
P<\mathbf{0 . 0 0 1}\end{array}$ & $\begin{array}{l}\mathrm{F}_{2,128}=3.461 \\
P=\mathbf{0 . 0 3 6}\end{array}$ \\
\hline Fall & $\sigma^{\prime}$ & $\begin{array}{l}\mathrm{F}_{1,113}=0.475 \\
P=0.495\end{array}$ & $\begin{array}{l}\mathrm{F}_{2,113}=37.424 \\
P<\mathbf{0 . 0 0 1}\end{array}$ & $\begin{array}{l}\mathrm{F}_{2,113}=3.264 \\
P=\mathbf{0 . 0 4 4}\end{array}$ \\
\hline Fall & $q$ & $\begin{array}{l}\mathrm{F}_{1,125}=3.462 \\
P=0.070\end{array}$ & $\begin{array}{l}\mathrm{F}_{2,125}=17.450 \\
P<\mathbf{0 . 0 0 1}\end{array}$ & $\begin{array}{l}\mathrm{F}_{2,125}=8.056 \\
P<\mathbf{0 . 0 0 1}\end{array}$ \\
\hline
\end{tabular}

\section{Effects of ACTH treatment on sex steroids}

We asked if ACTH-induced increases in glucocorticoids alter plasma androgen concentrations in males. There was a significant main effect of sampling time but not hormone treatment on androgens during both spring and fall (Table 2). There were no significant interactions between treatment and sampling time (Table 2). In springcollected males, a Student-Newman-Keuls multiple comparisons procedure revealed that plasma androgen concentrations decreased significantly at the $1 \mathrm{~h}$ sampling time (Fig. 2.2A). In fall-collected males, plasma androgen concentrations decreased significantly at the $4 \mathrm{~h}$ sampling time (Fig. 2.2B). Initial $(0 \mathrm{~h})$ androgen concentrations were significantly 
lower in spring compared to fall $(U=260.00$, d.f. $=1, P<0.001$; from a Mann-Whitney U-test), consistent with previously published data.

Estradiol concentrations were low or undetectable in most female snakes at all sampling times in both seasons (data not shown). We therefore did not perform any statistical tests on these data.

Table 2.2. Influence of adrenocorticotropic hormone (ACTH) on plasma androgens in
male red-sided garter snakes during the spring and fall.

\begin{tabular}{|llll|}
\hline Season & Treatment & Time & Interaction \\
\hline \multirow{2}{*}{ Spring } & $\mathrm{F}_{1,116}=0.007 ;$ & $\mathrm{F}_{2,116}=7.393 ;$ & $\mathrm{F}_{2,116}=0.386 ;$ \\
& $P=0.936$ & $P=\mathbf{0 . 0 0 1}$ & $P=0.681$ \\
\multirow{2}{*}{ Fall } & $\mathrm{F}_{1,107}=0.575 ;$ & $\mathrm{F}_{2,107}=14.878 ;$ & $\mathrm{F}_{2,107}=0.572 ;$ \\
& $P=0.454$ & $P<\mathbf{0 . 0 0 1}$ & $P=0.567$ \\
\hline
\end{tabular}

All statistics are from two-way repeated measures ANOVAs with treatment and sampling time as factors.

Integrated corticosterone response to ACTH treatment

To better evaluate how corticosterone responses to ACTH vary with season, sex and body condition, we calculated an integrated corticosterone response for each ACTHtreated snake and then compared these integrated responses using a non-parametric threeway ANOVA. Overall, there were no significant main effects of season $\left(H_{1,83}=2.293, P\right.$ $=0.130)$, sex $\left(H_{1,83}=2.977, P=0.084\right)$, or body condition $\left(H_{1,83}=0.756, P=0.385\right)$. There were no significant interactions between season and $\operatorname{sex}\left(H_{1,83}=3.169, P=0.075\right)$, season and body condition $\left(H_{1,83}=0.222, P=0.637\right)$ or sex and body condition $\left(H_{1,83}=\right.$ $0.174, P=0.677)$. However, there was a significant three-way interaction among season, sex, and body condition $\left(H_{1,83}=4.497, P=0.034\right)$. We noted a trend for ACTH treatment to elicit a larger integrated corticosterone response in males in positive body condition 
during the spring. Similarly, females in positive body condition collected during the fall also exhibited a larger response, although neither of these effects reached statistical significance (Fig. 2.3A).

Because of the significant interaction among season, sex, and body condition in our three-way ANOVA, we separated the data by sex and reanalyzed the male data to address one of our a priori questions: Does the sensitivity of male snakes to ACTH vary seasonally? A non-parametric two-way ANOVA with season and body condition as factors indicated that there was a significant main effect of season $\left(H_{1,37}=5.689, P=\right.$ $0.017)$ but not body condition $\left(H_{1,37}=0.137, P=0.712\right)$ on integrated corticosterone response to ACTH. The interaction between season and body condition $\left(H_{1,37}=3.091, P\right.$ $=0.079)$ was not statistically significant. These results indicate that males have a significantly smaller integrated corticosterone response to ACTH during the spring mating season compared to the fall (Fig. 2.3B).

To address our second a priori question (i.e., Are there sex differences in the sensitivity to ACTH during the spring?), we separated the data by season and reanalyzed the spring data using a non-parametric two-way ANOVA with sex and body condition as factors. Within the spring, there was a significant main effect of sex $\left(H_{1,42}=4.893, P=\right.$ $0.027)$ but not body condition $\left(H_{1,42}=0.594, P=0.441\right)$ on integrated corticosterone response to ACTH. There was not a significant interaction between sex and body condition $\left(H_{1,42}=1.003, P=0.317\right)$. Within the spring, males had a significantly smaller integrated corticosterone response to ACTH than females (Fig. 2.3B). 


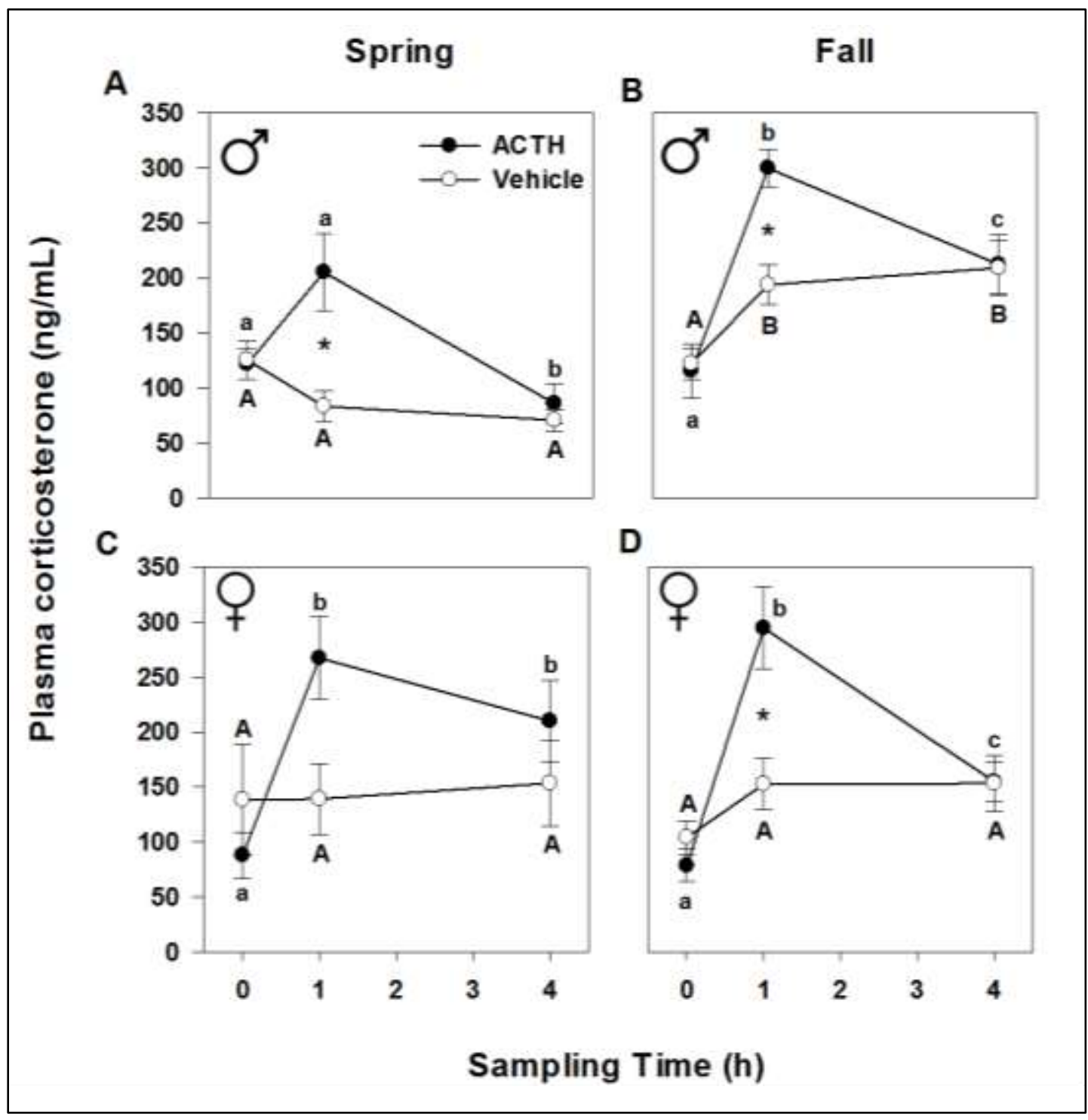

Figure 2.1. Effect of adrenocorticotropic hormone (ACTH) challenge on plasma corticosterone (mean \pm SE) in male and female red-sided garter snakes (Thamnophis sirtalis parietalis) during the spring mating season (A and C, respectively) and fall pre-dormancy period ( $\mathrm{B}$ and $\mathrm{D}$, respectively). Letters indicate significant differences among sampling times within ACTH-treated (lowercase) and vehicle-treated (uppercase) snakes. Asterisks indicate that corticosterone concentrations of ACTH-treated snakes are significantly higher than vehicle-treated snakes within the $1 \mathrm{~h}$ sampling time. All statistics are from a two-way repeated measures ANOVA within each sex and season followed by a Student-Newman-Keuls multiple comparisons test. Note the relatively smaller increase in corticosterone in response to ACTH treatment in males during the spring mating season. 


\section{Discussion}

We tested the hypothesis that observed variation in stress-induced glucocorticoid concentrations is related to the responsiveness of the adrenal glands to ACTH using an ACTH challenge. Overall, males collected during spring were less responsive to ACTH than males collected during fall or females in either season. These data explain, at least in part, why males are generally less sensitive to capture stress during the spring mating season. The corticosterone response to ACTH did not vary explicitly with body condition category, although we did observe a significant interaction among season, sex and body condition. Plasma androgens did not differ between vehicle- and ACTH-treated snakes. Taken together, our data elucidate a mechanism that explains how season, sex, and body condition affect glucocorticoid responses to capture stress: namely, variation in adrenal responsiveness to $\mathrm{ACTH}$.

\section{Seasonal differences in glucocorticoid responsiveness to ACTH challenge}

Because of the direct connection between sensitivity to ACTH and responses to stress, we predicted that snakes would be least responsive to ACTH when they are also least responsive to capture stress. Indeed, male red-sided garter snakes are least responsive to $\mathrm{ACTH}$ (this study) as well as capture stress during the spring compared to fall (Lutterschmidt and Mason, 2005; Moore et al., 2001, but also see Lutterschmidt and Mason, 2010; Moore et al., 2000a). We also found that vehicle-treated males during the fall increased corticosterone concentrations within 1 hour of injection, though to a lesser degree than ACTH-treated males, indicating that males during the fall pre-dormancy 
period are comparatively more sensitive to handling stress than spring-collected males.

Our data therefore support the hypothesis that decreased adrenal responsiveness to ACTH explains, at least in part, previous reports of suppressed stress responses in males during the mating season.

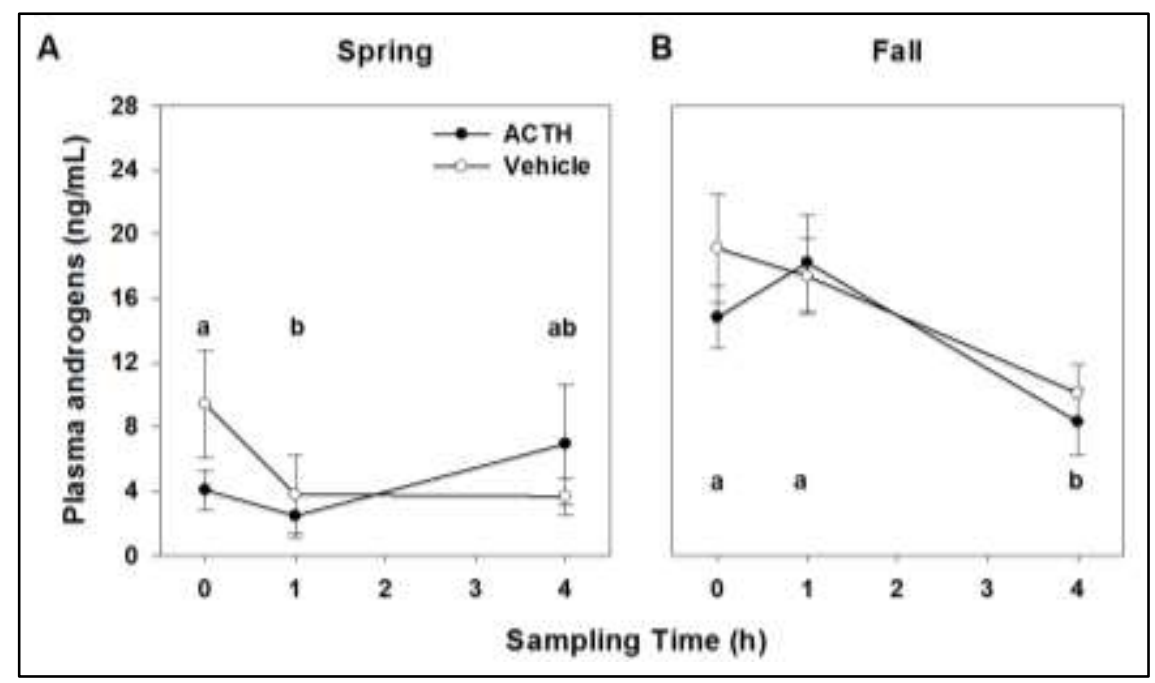

Figure 2.2. Influence of ACTH-induced changes in corticosterone on plasma androgens (mean $\pm \mathrm{SE}$ ) in male red-sided garter snakes (Thamnophis sirtalis parietalis) during the spring (A) and fall (B). Letters indicate significant differences among sampling times; ACTH treatment did not alter androgens. All statistics are from a two-way repeated measures ANOVA within each season followed by a Student-Newman-Keuls multiple comparisons test. Baseline $(0 \mathrm{~h})$ androgen concentrations were significantly lower during the spring compared to the fall.

Seasonal variation in responses to ACTH occurs in fish, amphibians, reptiles,

birds, and mammals (Astheimer et al., 1995; Capaldo et al., 2004; Cartledge and Jones, 2007; Girard et al., 1998; Gist, 1972; Ingram et al., 1999; Mashburn and Atkinson, 2008;

Romero and Wingfield, 1998; van Lier et al., 2003). Studies in birds and reptiles further suggest that modulation of sensitivity to ACTH is associated with plasticity in stress responses (e.g., Romero and Wikelski, 2006; Romero and Wingfield, 1998; Romero and Wingfield, 1999; Romero et al., 1998). One study in Galápagos marine iguanas 
(Amblyrhynchus cristatus) examined diel variation in the sensitivity to exogenous ACTH and capture stress and found that both treatments elicit larger corticosterone increases during the day versus the night (Romero and Wikelski, 2006). These data suggest that variation in ACTH responsiveness is a central mechanism used to modulate stress responses even on a daily basis.

Modulating responses to a stressor, whether by varying adrenal sensitivity to ACTH or by some other mechanism, may facilitate striking a balance between conflicting physiological processes. For example, elevated glucocorticoids can suppress nonessential processes like reproduction and digestion to promote immediate survival (reviewed in Landys et al., 2006; Wingfield and Sapolsky, 2003). However, the HPA axis may be adaptively regulated during certain time-sensitive life-history events to prevent elevated glucocorticoid concentrations from negatively impacting reproductive success (reviewed in Moore and Jessop, 2003; Romero, 2002; Wingfield and Sapolsky, 2003). In male red-sided garter snakes, elevated plasma corticosterone is associated with courtship behavior during the spring (Cease et al., 2007; Lutterschmidt and Maine, 2014), suggesting that energetically costly mating behavior relies on corticosterone to mobilize sufficient energy stores. When exogenous corticosterone is administered to males, however, courtship behavior is suppressed in a threshold-dependent manner (Lutterschmidt et al., 2004; Moore and Mason, 2001). Taken together, these data suggest that there is an optimal range of glucocorticoid concentrations during the mating season, below which insufficient energy is available to support courtship behavior, and above 
which glucocorticoids suppress reproduction (Lutterschmidt and Maine, 2014;

Lutterschmidt et al., 2004; Moore and Mason, 2001). By remaining within the optimal range of corticosterone concentrations, males likely maximize reproductive opportunities.

Overall, we interpret the seasonal difference in male responses to ACTH as a potentially adaptive modulation of the HPA axis to facilitate mating behavior and maximize reproductive success. Although we did not observe a seasonal difference in female responsiveness to $\mathrm{ACTH}$, whether female responses to capture stress vary seasonally as they do in males is unknown. If the magnitude of a stress response primarily depends upon adrenal sensitivity to ACTH, then we posit that female glucocorticoid responses to capture stress will also be similar across seasons, as are female responses to ACTH. These data will be critical for gaining a more comprehensive understanding of the seasonal regulation of the HPA axis.

Sex differences in glucocorticoid responsiveness to ACTH challenge

We observed a significant sex difference in responsiveness to ACTH during the spring mating season, such that males had a smaller corticosterone response to ACTH compared to females. Because female glucocorticoid responses to ACTH are consistently robust in both spring and fall, we suggest that the observed sex difference primarily reflects suppressed male responsiveness during the spring. Sex differences in the sensitivity of the HPA axis, particularly during the breeding season, have been reported previously (Ashley et al., 2011; Cartledge and Jones, 2007; Keenan et al., 2009; van Lier et al., 2003). For example, in male sheep (Ovis aries), ACTH elicits a smaller 
glucocorticoid response during the breeding season versus the non-breeding season, yet

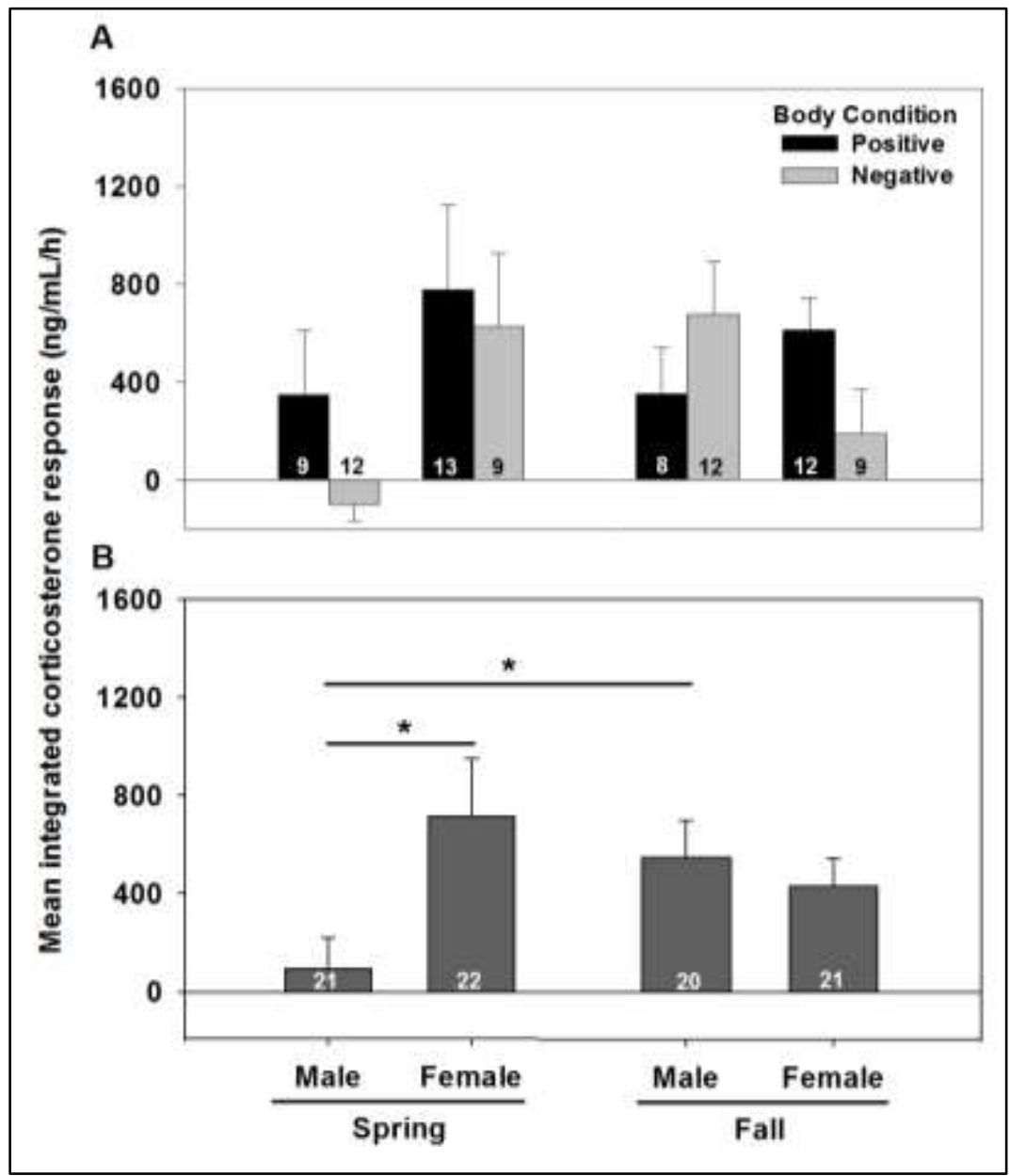

Figure 2.3. Mean integrated corticosterone response $(+1 \mathrm{SE})$ to adrenocorticotropic hormone (ACTH) challenge in male and female red-sided garter snakes (Thamnophis sirtalis parietalis) during the spring and fall (A). Body condition in panel A was calculated as the residual from a regression of body mass on snout-vent length. Because of a significant three-way interaction between season, sex and body condition, we reanalyzed the data to focus on two a priori questions: Are there sex differences during the spring?, and Does male sensitivity to ACTH vary seasonally? These comparisons are shown in (B) with body condition category collapsed for clarity (the effects of body condition were not statistically significant in either comparison). Within males, the asterisk indicates a significant seasonal difference in integrated corticosterone response (from a non-parametric two-way ANOVA on male data with season and body condition as factors). Within spring, the asterisk indicates a significant sex difference in integrated corticosterone responses to ACTH (from a non-parametric two-way ANOVA on spring data with sex and body condition as factors). Numbers along the abscissa are sample sizes in each category. 
female sheep respond to ACTH equally in both seasons (van Lier et al., 2003).

Female red-sided garter snakes exhibit decreased receptivity to mating in response to elevated glucocorticoids, both from exogenous corticosterone administration and capture stress treatment (Dayger et al., 2013). Female snakes retain ACTH responsiveness during the spring even though elevated glucocorticoids can suppress receptivity, suggesting that female snakes may not be as limited by stress-induced changes in reproductive behavior as males. Because the operational sex ratio is highly skewed towards males during the mating season, females have relatively more opportunities to mate than males. In addition, female red-sided garter snakes can utilize stored sperm from previous copulations for fertilization (Friesen et al., 2013; Halpert et al., 1982), and therefore females are not strictly obligated to copulate during a given mating season in order to reproduce. Thus, stress-induced suppression of mating behavior may have little impact on the overall reproductive success of females. If correct, this would sharply contrast with the effects of highly elevated glucocorticoids on male reproductive success, potentially explaining why reduced adrenal sensitivity to ACTH during the spring has evolved only in males. Further studies investigating the effects of different doses of ACTH are needed to better elucidate the nature of the observed sex difference in the sensitivity to ACTH during the spring mating season as well as its significance to reproductive success.

The influence of body condition on glucocorticoid responsiveness to ACTH

Overall, corticosterone responses to ACTH did not vary significantly with body 
condition, although we did observe a significant three-way interaction among season, sex and body condition. The analyses presented here focus on using body condition as a categorical variable for better comparison to previous studies (Cease et al., 2007; Dayger et al., 2013; Jessop et al., 2004; Moore et al., 2000b). However, we also did not observe any significant relationships between corticosterone responses to ACTH and body condition using regression analyses (data not shown). Our results therefore suggest that the influence of body condition on stress responsiveness (as reported by Dayger et al., 2013; Moore et al., 2000b) is subtle, and further research is needed to disentangle these effects from those of both season and sex. For example, there was a trend for ACTHtreated males in positive body condition during the spring to exhibit a larger corticosterone response than those in negative body condition; this trend may be related to the capacity to maintain the elevated corticosterone concentrations that support mating behavior (Cease et al., 2007; Lutterschmidt and Maine, 2014). In addition, fall-collected females in negative body condition tended to produce a smaller corticosterone response to ACTH than those in positive body condition. Because female red-sided garter snakes give birth every other year (Garstka et al., 1982; Gregory, 1977), females in negative body condition during the fall are most likely post-parturient. Similar to Lutterschmidt et al. (2009), the trend reported here may be related to modulation of HPA axis sensitivity in response to changing reproductive condition. The potential influence of body condition on stress response plasticity and the implications for reproductive outcomes in males and females requires further research in this and other taxa. 
Effects of ACTH challenge on sex steroids

Elevated plasma glucocorticoid concentrations can elicit a decline in androgen concentrations (Moore et al., 2000a; Moore et al., 2000b; reviewed in Rivier and Rivest, 1991), but this is not always the case (Cease et al., 2007; Lutterschmidt and Mason, 2005; Lutterschmidt et al., 2004; Moore and Mason, 2001). We observed a significant increase in glucocorticoid concentrations in response to ACTH treatment during both the spring and fall, yet androgen concentrations were unaffected compared to vehicle-treated snakes. The absence of an effect of ACTH treatment on androgens during the spring is not surprising, as red-sided garter snakes are temporally-dissociated breeders that have relatively low androgen concentrations during mating (reviewed in Lutterschmidt, 2012). Indeed, we observed relatively lower androgen concentrations in males during the spring compared to fall, as has been previously reported (e.g., Lutterschmidt and Mason, 2005; Lutterschmidt and Mason, 2009; Moore et al., 2001). Similar to Lutterschmidt and Mason (2005), we also did not observe a significant decrease in androgens in response to ACTHinduced glucocorticoid concentrations during the fall. However, male androgen concentrations significantly declined over the sampling period during both the spring and fall, regardless of treatment group. These results suggest that the observed decline in androgens is due to some other component of capture and handling that is not directly corticosterone dependent. For example, activation of the sympathetic nervous system can directly alter hormone synthesis and release independently of corticosterone treatment (e.g., Lutterschmidt and Mason, 2010). It is interesting to note that the time-course of these androgen changes varied between spring- and fall-collected males, suggesting that 
the mechanism mediating capture-stress induced changes in androgens, as yet undetermined, is also seasonally modulated.

While there have been several reports of elevated glucocorticoids influencing plasma estradiol concentrations in other female vertebrates (Clearwater and Pankhurst, 1997; Coddington and Cree, 1995; Elsey et al., 1991; Mahmoud et al., 1989; Shors et al., 1999; Woodley and Moore, 2002; Zerani et al., 1991), plasma estradiol concentrations are typically low or undetectable in unmated female red-sided garter snakes during the spring and in females collected during the fall pre-hibernation period (Garstka et al., 1985; Lutterschmidt and Mason, 2009; Uhrig et al., 2012; Whittier et al., 1987). Not surprising, plasma estradiol was undetectable in the majority of females in this experiment, and therefore any effects of ACTH treatment on estradiol concentrations could not be determined.

In summary, we observed seasonal and sex differences in the adrenal response to ACTH treatment in red-sided garter snakes. Here we present a mechanism explaining, at least in part, reduced stress sensitivity in male red-sided garter snakes during the spring breeding season (i.e., decreased responsiveness to ACTH). Females consistently exhibited a robust corticosterone response to ACTH that did not vary seasonally, suggesting that plasticity in adrenal sensitivity to ACTH is specific to males. Sex differences in the modulation of the HPA axis may therefore reflect differing pressures governing male and female fitness. Future research examining other mechanisms that alter HPA axis sensitivity, such as variation in negative feedback regulation of the HPA axis, are necessary to understand how the HPA axis facilitates the appropriate 
physiological and behavioral responses to a seasonally changing environment. Discovery of the processes that an animal uses to integrate internal and external cues will be crucial to understanding the fundamental mechanisms that impact reproductive success.

\section{Acknowledgements}

We thank the Manitoba Department of Conservation and Dave Roberts for logistical support in Manitoba, Canada. We thank Ashley R. Lucas and Robert T. Mason for their assistance in the field. 


\title{
Chapter 3
}

\section{Patterns of stress responses shift during seasonal life-history transitions: an analysis comparing baseline, maximal and integrated corticosterone in female red-sided garter snakes (Thamnophis sirtalis parietalis). Published in General and Comparative Endocrinology 246 (2017) 29-36}

\begin{abstract}
Glucocorticoids often rise and fall with a variety of external and internal cues and frequently vary among life-history stages. This suggests that changing glucocorticoids may coordinate life-history transitions. To explore this hypothesis, we asked if the timecourse of stress-induced glucocorticoid levels differ between two life-history transitions (i.e., spring and fall migration) in female red-sided garter snakes (Thamnophis sirtalis parietalis). We collected non-migratory females from a communal den and migratory females from a road along the migration route and treated them with $4 \mathrm{~h}$ of capture stress; plasma corticosterone was measured before, during and after capture stress. During the spring, den-collected females exhibited a stress-induced peak in corticosterone at an earlier sampling time than migrating, road-collected females. Because the pattern of corticosterone responses varied with migratory state, negative feedback on and/or sensitivity of the hypothalamus-pituitary-adrenal (HPA) axis may be linked to spring migration. During the fall, capture stress elicited an increase in corticosterone in dencollected females but not in migrating, road-collected females. Baseline corticosterone
\end{abstract}


was higher and both maximal and integrated corticosterone responses were lower during the fall compared to spring, indicating that stress responses are smaller when baseline corticosterone is elevated, perhaps due to a "ceiling effect." These data suggest that HPA axis regulation changes during seasonal migration, possibly via altering negative feedback, HPA axis sensitivity, or some other mechanism. This study supports the hypothesis that glucocorticoids coordinate life-history events and suggests that examining a suite of stress response characteristics is most informative for understanding the function of HPA modulation.

\section{INTRODUCTION}

Animals face high stakes if they fail to appropriately synchronize physiology and behavior with environmental conditions because this can impact survival and future reproductive fitness. While the factors associated with seasonal changes in physiology and behavior have been well studied, the mechanisms that induce an animal to transition between seasonal life-history stages remain poorly understood. Glucocorticoid hormones, regulated and secreted by the hypothalamus-pituitary-adrenal (HPA) axis, are prime candidates for coordinating seasonal life-history transitions because of their wide-ranging actions and the seasonal pattern of their release.

Because glucocorticoids mobilize energy stores, they have evolved as one of the

first systems to respond to both internal and environmental perturbations. Specifically, the HPA axis responds to perturbations (i.e., stressors) by increasing circulating glucocorticoid levels. Stress-induced glucocorticoids in turn coordinate a suite of physiological and behavioral changes that promote immediate survival, including 
suppressing reproduction and digestion and modulating metabolism and the immune system (Sapolsky et al., 2000). However, as a metabolic hormone, baseline glucocorticoids rise and fall in concert with the energetic demands of life-history stages (Landys et al., 2006; Romero, 2002). For instance, baseline glucocorticoids are elevated during reproduction and migration, two energetically demanding life-history stages, in white-crowned sparrows (Zonotrichia leucophrys). In some cases, elevated glucocorticoids are even vital for successful reproduction (Brann and Mahesh, 1991; Fanson and Parrott, 2015). For example, experimentally decreasing glucocorticoids suppresses female mating behavior in receptive female musk shrews (Suncus murinus) (Schiml and Rissman, 1999). Thus, current evidence suggests that glucocorticoids coordinate the physiological and behavioral changes necessary for animals to transition from one seasonal life-history stage to the next.

In addition to glucocorticoid actions being well-suited to regulate seasonal changes in physiology and behavior, virtually all aspects of HPA axis regulation and stress physiology have been shown to vary with season (Astheimer et al., 1995; Kitaysky et al., 1999; Klukowski, 2011; Lutterschmidt et al., 2009; Moore et al., 2001), sex (Cartledge and Jones, 2007; Pottinger et al., 2013; Wingfield et al., 1994), reproductive state (Cartledge and Jones, 2007; Kitaysky et al., 1999; Lutterschmidt et al., 2009; Schoech et al., 1997), body condition (Dayger et al., 2013; Heath and Dufty, 1998) and body size (Schmidt et al., 2012) in a variety of vertebrate systems. For example, baseline glucocorticoid concentrations vary seasonally in male bush warblers (Cettia diphone) and are higher at the peak of the breeding season in May than at the end of the breeding 
season in August (Wada and Shimizu, 2004). The magnitude of stress responses also varies seasonally in many taxa (Romero, 2002). Romero and Wingfield (1998) showed that adrenal responsiveness to ACTH was higher during the spring breeding season than during late winter migration in Gambel's white-crowned sparrows (Zonotrichia leucophrys gambelii). Further, corticosterone stress responses differed between Lapland longspurs (Calcarius lapponicus) that had just completed migration to the breeding grounds and those that had been at the breeding grounds for several weeks, supporting migration-related modulation of the HPA axis (Walker et al. (2015). These and other examples demonstrate that the activity and sensitivity of the HPA axis varies both seasonally and with life-history stage, thereby producing variation in plasma glucocorticoids that may, in part, mediate seasonal life-history transitions.

While there is precedence for glucocorticoids playing a role in seasonal lifehistory transitions, we do not yet understand if or how changes in HPA axis regulation coordinate the physiological and behavioral shifts necessary to accomplish these transitions. Moreover, the timing of life-history events is sexually dimorphic in many species (Ball and Ketterson, 2008), and glucocorticoids may play a role in regulating these sex differences. However, data comparing metrics of HPA axis regulation between sexes and during life-history transitions are lacking, thereby limiting our ability to evaluate the role of glucocorticoids in mediating these sexually dimorphic patterns.

In the current study, we asked if patterns of glucocorticoid responses to capture stress vary seasonally or with migration status in female red-sided garter snakes. Previous studies have documented such changes in male garter snakes (Cease et al., 2007; 
Lutterschmidt and Mason, 2005; Moore et al., 2001), but data regarding how season and migration status affect female stress responses do not exist. In both male and female snakes, baseline glucocorticoid concentrations are generally elevated during the spring mating season compared to the summer feeding season (Moore et al., 2001; Whittier et al., 1987). In males, Cease et al. (2007) reported that baseline glucocorticoids decline as snakes disperse from the den site and transition to feeding behavior. Similarly, Moore et al (2001) and Lutterschmidt and Mason (2005) reported seasonal variation in baseline glucocorticoid concentrations, with elevated levels occurring during the fall after snakes migrate back to the den site in preparation for hibernation. Hormonal responses to capture stress also vary seasonally in these northern populations of red-sided garter snakes (Lutterschmidt and Mason, 2005; Moore et al., 2001). Importantly, Dayger and Lutterschmidt (2016) showed that adrenal sensitivity to adrenocorticotropic hormone $(\mathrm{ACTH})$ varies seasonally and is sexually dimorphic. Taken together, these data suggest that seasonal changes in HPA axis activity and/or sensitivity may in fact play a role in mediating observed sex differences in the timing of migration in this species.

To better understand the nature and extent of sex differences in HPA axis activity, we investigated whether responses to a standardized capture stress protocol varied with season or migratory status in female red-sided garter snakes. Much of the literature investigating the regulation of the HPA axis is focused on male animals, overlooking potential sex differences. These data in female red-sided garter snakes will permit comparisons with the existing body of literature on responses of male snakes to capture stress and aid in identifying potential sexual dimorphisms. Previous studies in this and 
other species (e.g., Anderson et al., 2014; Bauer et al., 2014; Dayger et al., 2013; Moore et al., 2001) have demonstrated that changes in HPA axis activity and/or sensitivity can be assessed by examining the time-course of glucocorticoid responses to capture stress. To better understand if and how patterns of glucocorticoid responses to capture stress vary with season and migration status in female snakes, we compared results obtained from analyses of baseline glucocorticoids, maximal stress-induced responses, and integrated corticosterone responses to capture stress.

\section{METHODS}

\subsection{Experimental design}

Female red-sided garter snakes were collected from a den site in Inwood, Manitoba, Canada and from a road along the snakes' migration route during the spring mating season from 13-18 May 2012 and during the fall pre-hibernation period from 1012 September 2012. As in Cease et al. (2007), the rural road is located approximately 1 $\mathrm{km}$ from the den site and was used to aid in intercepting migrating snakes. Snakes generally pass over the road quickly and do not linger near the road. Females collected at the den during the spring were confirmed to be unmated by checking for the absence of a copulatory plug in the cloaca. Because copulatory plugs can degrade and fall out during dispersal (Shine et al., 2000), all dispersing females were assumed to have mated prior to reaching the road. Final sample sizes were 26 and 14 females collected during the spring from the den and road, respectively, and 17 and 17 females collected during the fall from the den and road, respectively. Note that the corticosterone data obtained from female 
snakes collected from the den site during the spring mating season were reported in Dayger et al. (2013) as part of an independent analysis. In that study, the data were used to determine if female responses to capture stress during the spring mating season varied with body condition. In the current study, these data are being included to determine if stress responses vary specifically with season and migratory status.

\subsection{Blood collection and capture stress treatment}

Immediately upon capture, a blood sample (approximately $150 \mu \mathrm{L}$ ) was collected from the caudal vein of each snake within 2 min of capture using $1 \mathrm{cc}$ syringes and 25 gauge needles. Snakes were placed individually into small, opaque canvas bags (approximately $20 \mathrm{~cm}$ x $20 \mathrm{~cm}$ ) for $4 \mathrm{~h}$ to induce a stress response. Additional blood samples were collected from each snake during $(2 \mathrm{~h})$ and after $(4 \mathrm{~h})$ capture stress treatment. All blood samples were stored on ice until returning to the research station, where each sample was centrifuged. All plasma samples were stored at $-20^{\circ} \mathrm{C}$ until being transported to Portland State University on dry ice, where they were stored at $-80^{\circ} \mathrm{C}$ until analysis.

\subsection{Radioimmunoassay}

Plasma samples were assayed for corticosterone, the primary glucocorticoid in reptiles, by direct radioimmunoassay following methods previously described by Dayger et al. (2013) and Dayger and Lutterschmidt (2016) and validated by Lutterschmidt and Mason $(2009,2005)$. Briefly, steroid hormones were extracted from 5-20 $\mu \mathrm{L}$ of plasma 
with anhydrous ethyl ether. The solvent phase was collected and evaporated at $37^{\circ} \mathrm{C}$ under nitrogen gas and the extracted hormones were reconstituted in phosphate-buffered saline. Each sample was analyzed in duplicate by incubating with $12,000 \mathrm{cpm} \mathrm{1,2,5,7-}$ $\left[{ }^{3} \mathrm{H}\right]$ corticosterone (Product number: NET399250UC; Perkin Elmer, Piscataway, NJ, USA) and $100 \mu \mathrm{L}$ corticosterone antibody (Product number: 07120016; MP Biomedicals, LLC, Solon, OH, USA) at $4^{\circ} \mathrm{C}$ for 16-20 h. Unbound hormone was separated from bound corticosterone with dextran-coated charcoal and the radioactivity of each sample was quantified using a liquid scintillation counter (Beckman 6500, Beckman Coulter, Inc., Brea, CA, USA). Samples were analyzed in 3 assays with an inter-assay coefficient of variation of $15.40 \%$ and a mean intra-assay coefficient of variation of $15.43 \%$. Measurements were corrected for individual recovery variation and the mean percent recovery was $72.95 \%$. The mean limit of detectability was $22.05 \mathrm{ng} / \mathrm{mL}$.

\subsection{Data analyses}

All data were confirmed to meet the assumptions of normality and equal variance required for parametric tests using a Shapiro-Wilk test for normality and Levene's mean test for equal variance. If transformation could not correct for deviations from normality, a non-parametric alternative was used. Statistical tests were considered to be significant at $P \leq 0.05$. All significant main effects revealed by our analyses were further investigated using Student-Newman-Keuls multiple comparisons tests. SigmaPlot 12.0 (Systat Software 2010, Systat Systems, Inc., Point Richmond, CA, USA) and JMP 12.0 (SAS Institute, Inc, Cary, NC, USA) were used for all statistical analyses. 
To examine if the temporal pattern of stress responses change seasonally or with migration status, we first used a one-way repeated measures analysis of variance (ANOVA) with sampling time as the within-subjects factor. Data from each season and location were analyzed separately to increase statistical power and ensure that subtle changes in corticosterone over time could be detected. For these analyses, the corticosterone data were additionally confirmed to meet the assumption of sphericity using a Mauchly's sphericity test. In one instance (i.e., corticosterone data from roadcollected females during spring), the data did not meet the assumption of sphericity. In this case, a Greenhouse-Geisser adjusted univariate F-test was used with sampling time as the within-subjects factor.

Because the pattern of stress-induced corticosterone release over time differed between seasons and with migratory status, we next asked if HPA axis activity and/or sensitivity to stress contributed to these patterns. Specifically, we asked if these differences were due primarily to changes in baseline concentrations, maximal stressinduced concentrations, or integrated corticosterone responses to capture stress. Baseline corticosterone was determined as the initial corticosterone concentration measured at time 0 for each snake. The maximal corticosterone response was calculated for each individual by subtracting the initial (baseline) corticosterone concentration from the highest corticosterone concentration reached during the capture stress treatment. The integrated response represents the total change in corticosterone from baseline during the entire sampling period (Cockrem and Silverin, 2002; Dayger and Lutterschmidt, 2016; Rensel and Schoech, 2011). To determine this value, we plotted corticosterone 
concentration against time and calculated the area under the curve for each individual.

We corrected for individual variation in baseline concentrations by multiplying the initial $(0 \mathrm{~h})$ corticosterone concentration by $4 \mathrm{~h}$ to account for baseline concentrations during the entire sampling period; this value was then subtracted from the total response to yield the corrected integrated corticosterone response (Fig. 3.1).

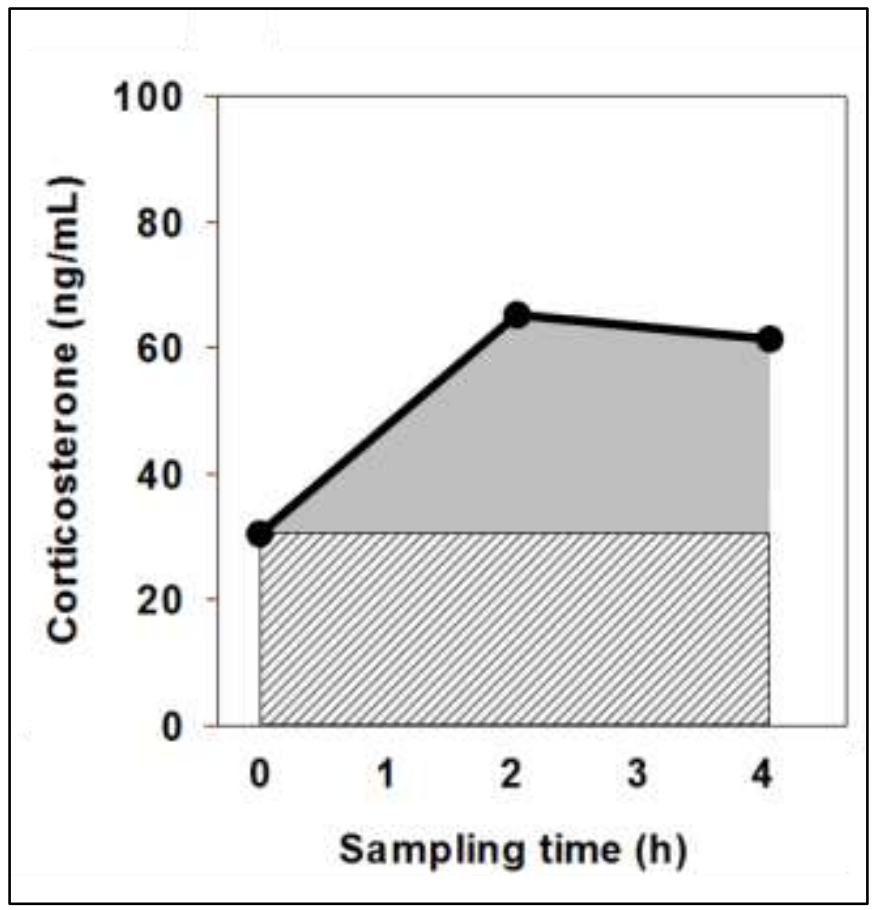

Figure 3.1. Calculation of the integrated corticosterone response. Corticosterone concentrations were plotted against time (solid black line). The total integrated corticosterone response was then calculated as the area under the solid black line for each individual. Individual variation in baseline concentrations was corrected for by multiplying the initial $(0 \mathrm{~h})$ corticosterone concentration by $4 \mathrm{~h}$ (hashed rectangle) to account for baseline concentrations during the entire sampling period; this value was then subtracted from the total response to yield the corrected integrated corticosterone response (gray polygon).

Potential differences in baseline corticosterone were examined using a two-way ANOVA with season and migration status as between-subjects factors; data were square 
root-transformed to meet the assumption of normality. In the maximal and integrated corticosterone data, transformation could not correct for deviations from normality, and thus non-parametric two-way ANOVAs were performed using the Scheirer-Ray-Hare extension of the Kruskall-Wallis ANOVA; season and migration status were included in these analyses as between-subjects factors.

\section{RESULTS}

\subsection{Temporal patterns of corticosterone responses to capture stress}

During the spring mating season, we found a significant main effect of sampling time on corticosterone responses to capture stress in both den-collected $\left(\mathrm{F}_{2,77}=5.624, P\right.$ $=0.006)$ and road-collected (Greenhouse-Geisser adjusted $\left.F_{2,41}=9.231, P=0.004\right)$ females. The pattern of stress-induced corticosterone varied with migration status (Fig. 3.2A-B). Den-collected females had significantly elevated plasma corticosterone at the 2 h sampling time that returned to baseline by the $4 \mathrm{~h}$ sampling time, while migrating, roadcollected females showed a significant increase in corticosterone only at the 4 h sampling time. During fall migration back to the den site, road-collected females did not significantly change corticosterone concentrations in response to capture stress at any sampling time $\left(\mathrm{F}_{2,50}=0.518, P=0.601\right.$, Fig. 3.2D). However, females collected from the den after completing the fall migration responded to capture stress with a significant increase in corticosterone concentrations at the $4 \mathrm{~h}$ sampling time $\left(\mathrm{F}_{2,50}=4.144, P=\right.$ 0.025, Fig. 3.2C). 


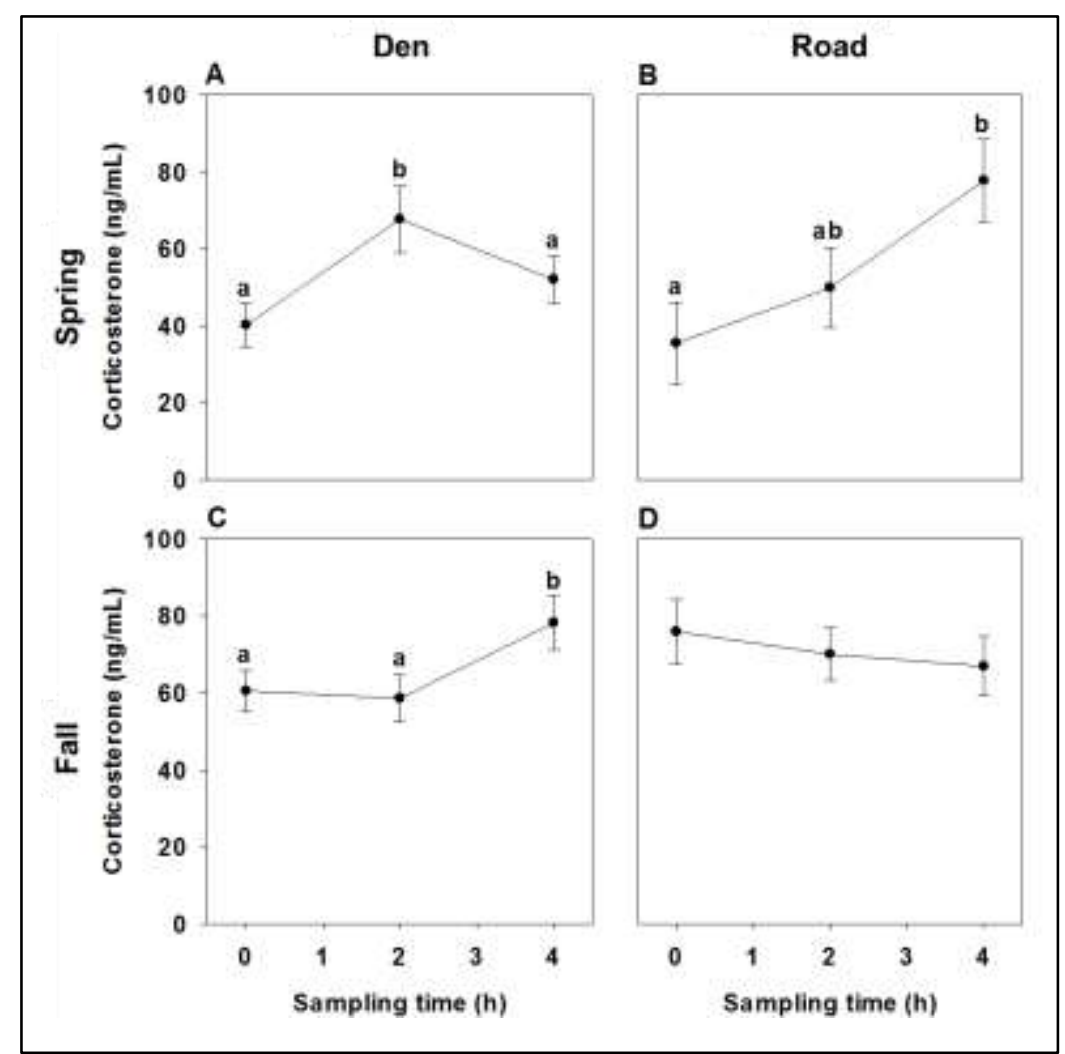

Figure 3.2. Corticosterone responses to capture stress $( \pm$ SEM) in female red-sided garter snakes (Thamnophis sirtalis parietalis) collected from the den and road during the spring mating season $(A, B$, respectively) and fall pre-dormancy period $(C, D$, respectively). Lowercase letters indicate significant differences in corticosterone concentrations among sampling times within each panel. Sample sizes during the spring were $\mathrm{n}=26$ collected from the den and $\mathrm{n}$ $=14$ from the road. During the fall, sample sizes were $\mathrm{n}=17$ and $\mathrm{n}=17$ collected from the den and road, respectively.

\subsection{Understanding why temporal patterns vary}

Based on the variation we observed in the patterns of corticosterone responses to capture stress, we examined whether baseline concentrations, maximal stress-induced concentrations, or integrated corticosterone responses to capture stress varied with season and/or migration status. We found a significant main effect of season $\left(\mathrm{F}_{1,73}=22.598, P<\right.$

$$
0.001) \text { but not migration status }\left(\mathrm{F}_{1,73}=0.020, P=0.888\right) \text { on baseline corticosterone }
$$

concentrations, with no significant interaction between factors $\left(\mathrm{F}_{1,73}=2.039, P=0.158\right.$; 
Fig. 3.3A). Females had significantly higher baseline plasma corticosterone during the fall pre-hibernation period than the spring mating season, regardless of migration status. Maximal corticosterone responses differed between spring and fall $\left(\mathrm{H}_{1,73}=7.086, P=\right.$ $0.008)$, but not with migration status $\left(\mathrm{H}_{1,73}=0.056, P=0.814\right)$. We found no significant interaction between season and migration status $\left(\mathrm{H}_{1,73}=1.994, P=0.158\right.$; Fig. 3.3B $)$.

Similarly, we found a significant main effect of season $\left(\mathrm{H}_{1,73}=8.428, P=0.004\right)$ but not migration status $\left(\mathrm{H}_{1,73}=0.053, P=0.817\right)$ on integrated corticosterone responses to capture stress (Fig. 3.4). There was no significant interaction between factors $\left(\mathrm{H}_{1,73}=\right.$ $0.302, P=0.583)$. For both maximal and integrated corticosterone responses, females collected during the spring mating season had higher responses than those collected during the fall pre-hibernation period.

\section{DISCUSSION}

We examined if glucocorticoid stress responses differed with season or migration status to determine if regulation of the HPA axis changes as snakes transition between seasonal life-history stages. Capture stress stimulated a significant increase in corticosterone during the spring, although the peak concentration occurred at different sampling times in den- and road-collected females. During the fall, stress treatment did not increase corticosterone in migrating females, although once females reached the den, there was a stress-induced corticosterone increase. These results suggest that regulation of the HPA axis changes as snakes migrate to and from the summer feeding grounds. Indeed, we found higher baseline corticosterone concentrations during the fall compared 


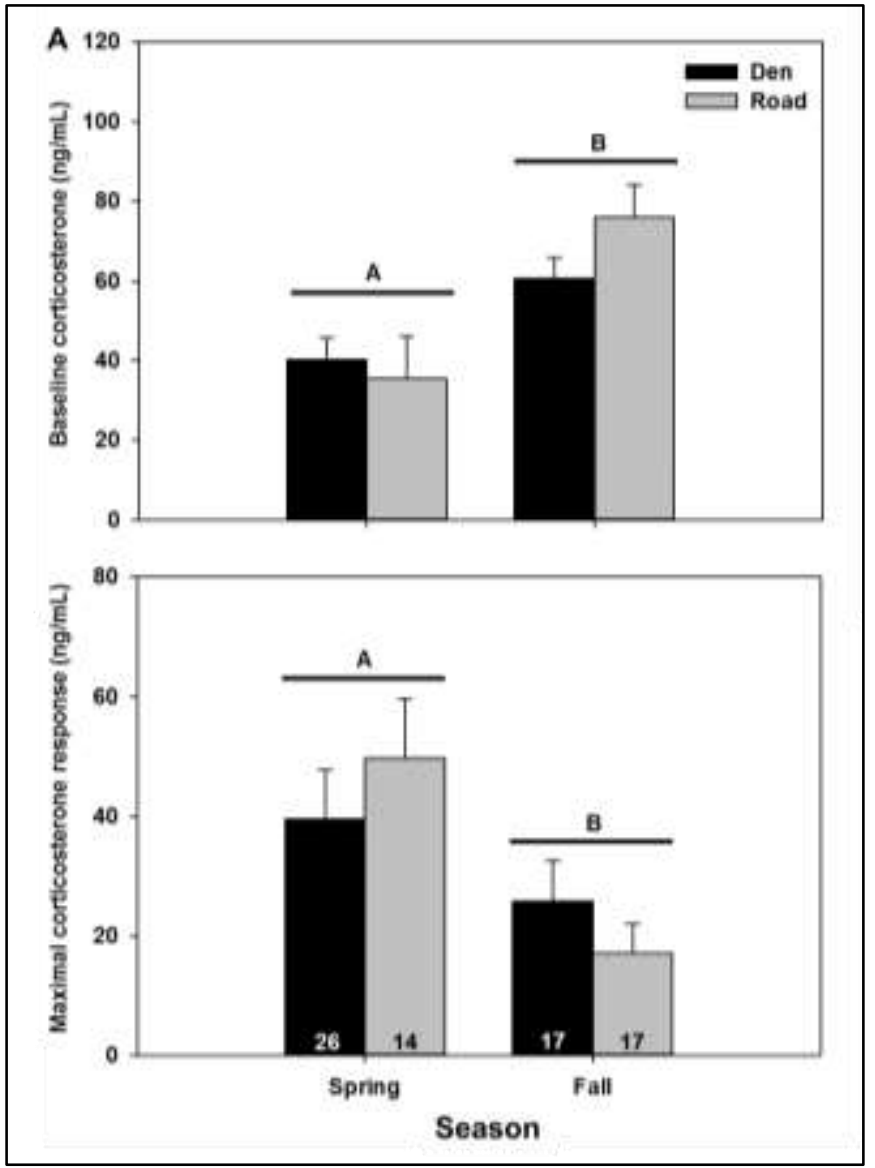

Figure 3.3. Baseline ( 0 h) corticosterone concentrations (A) and maximal corticosterone responses to capture stress (B) in female red-sided garter snakes (Thamnophis sirtalis parietalis) collected from the den and road during the spring and fall. Data are the mean + 1 SEM. Letters indicate significant seasonal differences in corticosterone concentrations. Numbers along the abscissa are sample sizes in each group.

to the spring, suggesting that elevated baseline corticosterone may be important for fueling the fall migration to the den site and coordinating physiological preparation for hibernation. In contrast, maximal and integrated corticosterone responses to capture stress were significantly lower during the fall compared to the spring. Similar to previous studies in male red-sided garter snakes, our results in female snakes suggest that when baseline corticosterone is already high, capture stress cannot significantly increase 
corticosterone levels further (Cease et al., 2007; Karten et al., 2005; Moore et al., 2001). By comparing the current data in females with previous reports in males, we noted sex differences in migration-related changes in glucocorticoid stress responses. Taken together, these data indicate that regulation of the HPA axis changes significantly as female snakes transition between seasonal life-history stages.

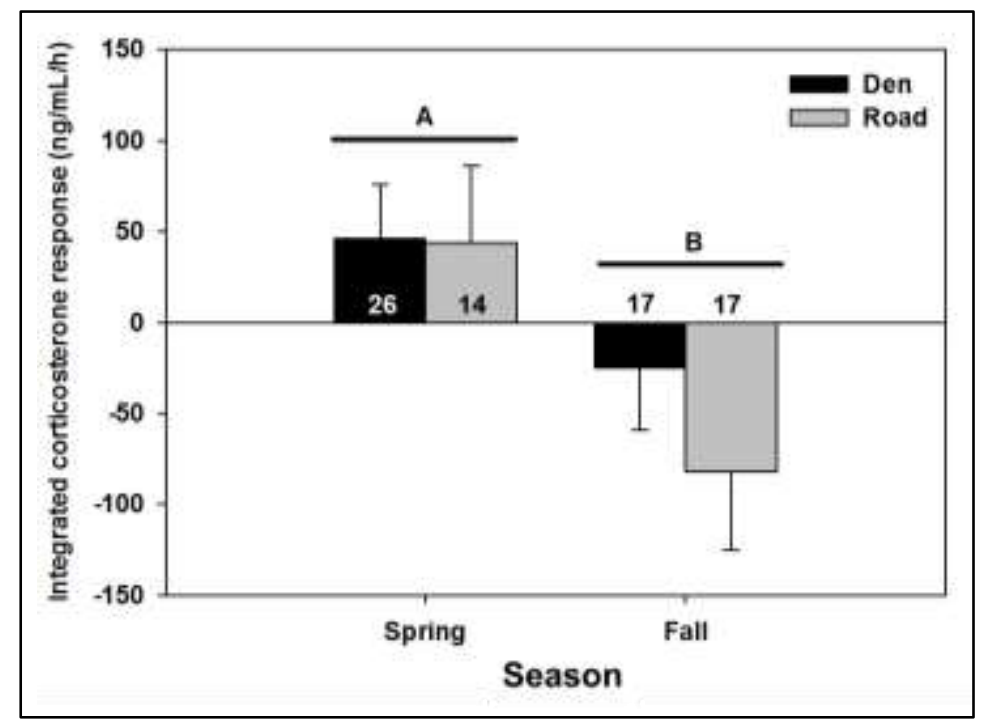

Figure 3.4. Integrated corticosterone responses to capture stress $( \pm$ SEM) in female red-sided garter snakes (Thamnophis sirtalis parietalis) collected from the den and road during the spring and fall. Letters indicate significant seasonal differences in integrated corticosterone responses. Numbers along the abscissa are sample sizes in each group.

\subsection{Seasonal variation in glucocorticoids}

We observed a seasonal difference in the pattern of stress-induced corticosterone release in females collected from the den site: during the spring, the peak stress-induced corticosterone level occurred at the $2 \mathrm{~h}$ sampling time, while during the fall, the peak occurred at the $4 \mathrm{~h}$ sampling time, indicating that spring-collected females responded to capture stress more quickly. The variation in corticosterone responses we observed with season could be due to several factors. Fall females might be less sensitive to capture 
stress and take longer to mount a response, perhaps due to differences in adrenal sensitivity to ACTH, as has been reported in many taxa (Astheimer et al., 1995; Capaldo et al., 2004; Cartledge and Jones, 2007; Girard et al., 1998; Gist, 1972; Ingram et al., 1999; Mashburn and Atkinson, 2008; Romero et al., 1998; Romero and Wingfield, 1998; van Lier et al., 2003). However, Dayger and Lutterschmidt (2016) found no seasonal differences in adrenal sensitivity to ACTH in female red-sided garter snakes collected from the den site during the spring and fall, although only one ACTH dose was tested in that study. It is possible that strong negative feedback on the HPA axis dampened the corticosterone stress response in den-collected females during the spring. Circulating glucocorticoids feed back on the HPA axis to suppress their own release (Whitnall, 1993). Lattin et al. (2012a) reported that negative feedback was stronger in house sparrows (Passer domesticus) during the breeding season compared to the post-breeding molting season. Similar to the pattern observed in house sparrows, the strength of negative feedback on the HPA axis may be stronger when females are at the breeding grounds during the spring and then weaken as females transition to migratory behavior and disperse to the summer feeding grounds. Significantly, strong negative feedback would allow animals with limited breeding opportunities to reproduce despite the presence of environmental perturbations and elevated glucocorticoids. Note that changing sensitivity of the HPA axis to ACTH and negative feedback are not mutually exclusive, and it is likely that a combination of these and other mechanisms are central to coordinating seasonal life-history transitions.

Baseline corticosterone concentrations were significantly lower during the spring 
compared to fall. Thus, capture stress elicited a larger maximal and integrated corticosterone response during the spring even though the peak concentrations were similar between seasons. Conversely, females had higher baseline corticosterone and smaller stress responses during the fall, suggesting that elevated baseline corticosterone could be an important component of preparing for winter dormancy, as hypothesized for male garter snakes by Lutterschmidt and Mason (2005). Elevated pre-hibernation glucocorticoid concentrations have been reported in hibernating mammals and have been suggested to play a role in the metabolic changes that facilitate pre-hibernation fattening (Armitage, 1991; Gustafson and Belt, 1981; Reeder et al., 2004; Shivatcheva et al., 1988). However, baseline corticosterone was also elevated during the fall migration back to the den site, and therefore whether elevated glucocorticoids are related to migratory behavior, preparation for winter dormancy, or both, requires further study.

\subsection{Variation in glucocorticoids related to migration status}

Owen et al. (2014) found that copperhead snakes (Agkistrodon contortrix) living near roads had lower maximum stress-induced corticosterone levels than forest-dwelling snakes. These results suggest that life at the road is associated with blunted stress responses in copperheads, perhaps due to traffic on the roads (Owen et al., 2014). Current data indicate that a similar relationship does not exist in this population of red-sided garter snakes. In this study, for example, female garter snakes collected from the den and road during spring had similar baseline and maximum corticosterone concentrations. Cease et al. (2007) also reported lower baseline but similar stress-induced corticosterone in male snakes collected from the road. Finally, we observed a seasonal difference in 
stress responses at the road in this study, suggesting that proximity to the road is not associated with any particular HPA axis response. All red-sided garter snakes must pass over the roads surrounding the food-limited den site to reach the necessary prey resources at the marshy feeding grounds (Gregory and Stewart, 1975). Thus, the population of redsided garter snakes we studied does not live either near or far from the road, but rather experiences roads briefly and seasonally as part of their migration events. Together, these observations suggest that differences in HPA axis activity between red-sided garter snakes collected from the den and road are not related to the road environment per se, but rather are most likely associated with the physiological changes that coincide with migration.

During the spring, females collected from the den had a different pattern of stressinduced corticosterone release than females collected from the road, with den-collected females experiencing a peak at the $2 \mathrm{~h}$ sampling time and migrating, road-collected females peaking at $4 \mathrm{~h}$. These data indicate that in den-collected females, plasma corticosterone responded to capture stress and returned to baseline more quickly than in road-collected females. Importantly, these migration-related differences during the spring (Fig. 3.2A vs. 3.2B) mirrored the spring-fall differences in the stress responses of nonmigrating females (Fig. 3.2A vs. 3.2C). These results suggest that seasonal changes in HPA axis regulation are initiated as soon as females begin to migrate from the spring breeding grounds. Our data also suggest that the glucocorticoid responses exhibited by den-collected females during spring are unique among the sampling times we tested and could be specifically related to breeding activity. 
Similar migration-related changes in the pattern of corticosterone stress responses have been reported in an artic-breeding bird, the Lapland longspur (Calcarius lapponicus). Female Lapland longspurs experience peak stress-induced corticosterone at an earlier sampling time during the breeding season than when sampled during migration (Astheimer et al., 1995). Differences in HPA axis activity between spring and fall migration occur in other bird species as well (Eikenaar et al., 2015; Raja-aho et al., 2013; Romero et al., 1997). Together, these studies suggest that migration-related changes in the stress response may be a conserved function of the HPA axis across taxa, but further research will be required to test this hypothesis.

During fall migration, females had the highest baseline corticosterone of any of the groups and experienced no significant stress-induced corticosterone increase. The migration modulation hypothesis, first proposed in birds, describes a similar migrationspecific pattern of glucocorticoid release characterized by elevated baseline corticosterone and the lack of a significant stress-induced increase in corticosterone (Holberton et al., 1996). Our findings in female snakes during fall migration, but not spring migration, support the migration modulation hypothesis. Similar differences in the stress responses of yellow-rumped warblers (Dendroica coronata) during spring and fall migration were reported by Holberton (1999). The authors suggested that the variation resulted from seasonal differences in energetic condition because birds were leanest during spring migration and fattest during fall migration. As in yellow-rumped warblers, male red-sided garter snakes also had a lower body condition index during the spring compared to fall (Moore et al., 2001), and therefore seasonal differences in stress 
responses could be related to seasonal differences in energetic condition. For example, during the spring, female red-sided garter snakes with a low, but not high, body condition index responded to capture stress with a significant increase in corticosterone (Dayger et al., 2013). These data support the hypothesis that seasonal changes in HPA axis activity may be linked to seasonal changes in energetic condition in both male and female redsided garter snakes.

Baseline concentrations, maximal stress-induced corticosterone, and the integrated corticosterone response did not vary with migration status, indicating that it is the pattern of corticosterone release, rather than the magnitude of the response or the total change in corticosterone, that varies during life-history transitions. These data highlight that the timing and duration of the stress-induced corticosterone response can be just as, if not more, informative for understanding the context-dependency of stress responses than the magnitude alone. Indeed, examining the temporal pattern of corticosterone responses can elucidate the mechanisms that modulate the activity of the HPA axis. For example, in Galapagos marine iguanas (Amblyrhynchus cristatus) that later died or survived an El Niño event, baseline glucocorticoids, stress-induced glucocorticoids and adrenal responsiveness to ACTH were similar in all iguanas (Romero and Wikelski, 2010). However, compared to the iguanas that later died, iguanas that survived the El Niño event had stronger negative feedback regulation of the HPA axis, resulting in stressinduced glucocorticoid concentrations returning to baseline more quickly. Genetic variation may underlie differences in HPA axis activity and sensitivity among individuals and may be a target of natural selection (Muráni et al., 2010; Schwartz and Bronikowski, 
2013). As such, the way the HPA axis responds within a particular context (e.g., season, sex, or life-history stage) may also be subject to selection pressure. Our data demonstrate that multiple measures of stress responsiveness, including baseline and maximal hormone concentrations, the time-course of the hormone response, and the cumulative change in hormone concentrations, provide a more accurate assessment of HPA axis modulation. Such data are necessary to fully understand the adaptive nature of stress responses as well as how context-dependent activity of the HPA axis evolved.

\subsection{Sex differences in glucocorticoids}

This study is the first seasonal comparison of stress responses in female red-sided garter snakes. With the opportunity to compare our data in females to previous studies in males, we can now uncover potential sex differences. Following a capture stress methodology identical to the one employed in our study, den-collected males in the spring do not respond to capture stress with a significant increase in corticosterone over baseline levels (Cease et al., 2007; Moore et al., 2001), although studies using different sampling regimes yielded different results (Lutterschmidt and Mason, 2010, 2005; Moore et al., 2000). The influence of the capture stress protocol and the time-course of sample collection on stress responses requires further examination to confirm the sex difference implicated by our new results: that den-collected females remain sensitive to capture stress during the spring mating season, while males have reduced stress sensitivity. In support of this observation, males are significantly less responsive to ACTH during the spring than females (Dayger and Lutterschmidt, 2016). This sex difference may also be 
present during the fall, as the den-collected females in this study, but not males (Lutterschmidt and Mason, 2005; Moore et al., 2001), increased corticosterone in response to capture stress. However, adrenal sensitivity to ACTH did not vary with sex during the fall (Dayger and Lutterschmidt, 2016). Therefore, different mechanisms, such as variation in the release of corticotropin releasing hormone $(\mathrm{CRH})$, adrenal ACTH release in response to $\mathrm{CRH}$, corticosteroid-binding globulin levels or activity, glucocorticoid receptor density, and negative feedback on the HPA axis could explain sex differences during the fall. All of these mechanisms have been shown to vary seasonally in at least some birds (e.g., Breuner and Orchinik, 2001; Lattin et al., 2016, 2012b; Liebl et al., 2013; Romero and Wingfield, 1998, 1998). During spring migration, both males and females exhibited significant stress responses, but baseline corticosterone varied with migration status in males (Cease et al., 2007). No studies have investigated male stress responses during fall migration, and thus sex differences during fall migration cannot be determined. Note that there is considerable annual variation in male baseline corticosterone concentrations and stress responses (Lutterschmidt and Mason, 2005), and without multiple years of female data, it is unknown whether females show similar variation.

\section{CONCLUSIONS}

In summary, we report that both baseline glucocorticoid concentrations, maximal stress-induced concentrations, and integrated corticosterone responses to capture stress varied seasonally in female red-sided garter snakes. Additionally, the time-course of the stress response differed between nonmigratory and migratory females suggesting that 
negative feedback on the HPA axis and/or adrenal sensitivity to ACTH may change as females transition to migratory behavior. By comparing the current study with data from male red-sided garter snakes, we note possible sex differences in glucocorticoid stress responses, alluding to the potential for further sex differences in HPA axis regulation as snakes transition between life-history stages. Further research directed at how regulation

of the HPA axis shifts during life-history transitions will be valuable for understanding if and how glucocorticoids mediate the physiological and behavioral changes that are critical to each life-history stage.

\section{ACKNOWLEDGEMENTS}

We thank the Manitoba Department of Conservation and Dave Roberts for logistical support in Manitoba, Canada and Ashley R. Lucas for assistance in the field. Partial funding was provided by a Student Research Grant from the Animal Behavior Society and a Forbes-Lea Award from Portland State University to C.A.D. 


\title{
Chapter 4
}

\section{Responses to capture stress and exogenous corticosterone vary with body condition in female red-sided garter snakes (Thamnophis sirtalis Parietalis)}

Published in Hormones and Behavior 64(4) 748-754

\begin{abstract}
This study examined whether hormonal and behavioral responses to capture stress and exogenous corticosterone (CORT) vary with body condition in female red-sided garter snakes (Thamnophis sirtalis parietalis). Female snakes were collected during the spring mating season and treated with $4 \mathrm{~h}$ of capture stress. We measured plasma CORT and estradiol before, during and after capture stress treatment followed by latency to copulate, a measure of female receptivity. Body condition was determined as the residual from a regression of body mass on snout-vent-length. Baseline CORT did not differ between females in positive and negative body condition, but females in negative body condition showed a significantly larger increase in plasma CORT in response to capture stress. Estradiol, which is generally low during the mating season in this population, did not change in response to capture stress. Body condition, but not capture stress, influenced latency to copulate, suggesting that females are resistant to the behavioral effects of capture stress during the spring mating season. In a second experiment, only females in negative body condition increased latency to copulate in response to injection of a physiological $(15 \mu \mathrm{g})$ dose of exogenous CORT, while all females responded to a
\end{abstract}


pharmacological $(60 \mu \mathrm{g})$ dose. These results indicate that behavioral responses to exogenous CORT vary with female body condition during the short mating season. Taken together, our data suggest that variation in body condition may be associated with differences in HPA axis sensitivity and/or glucocorticoid receptor density in the brain.

\section{INTRODUCTION}

Animals respond to changes in their external and internal environment by modulating the activity of the hypothalamus-pituitary-adrenal (HPA) axis, causing a change in the secretion of adrenal glucocorticoids (Wingfield and Ramenofsky, 1999). Unpredicted challenges to energy homeostasis, such as unusually low environmental temperatures and food shortage stimulate increases in plasma glucocorticoids (Harvey et al., 2005; Heath and Dufty, 1998; Wingfield, 1988). Canonically, increases in circulating glucocorticoids suppress non-essential functions like reproduction, prompting a transition into what is sometimes referred to as the "emergency life history stage" (Wingfield et al., 1998). However, a growing body of evidence suggests that hormonal and behavioral responses to glucocorticoids are highly context-dependent (reviewed in Moore and Jessop, 2003; Romero, 2002). For example, in female side-blotched lizards (Uta stansburiana), exogenous glucocorticoid treatment in one morph increased survival and reproduction while the same treatment in a second morph produced the opposite effects (Lancaster et al., 2008).

Variation in HPA axis sensitivity may be one mechanism by which context is coded by glucocorticoids. For example, variation in baseline glucocorticoid levels often 
accompanies changes in stress responsiveness and may reflect differences in HPA axis sensitivity (reviewed in Landys et al., 2006). One important cue integrated via glucocorticoids is internal energy balance. Failing to correctly allocate available energy stores to life history processes such as feeding, predator-avoidance, and reproduction can result in decreased reproductive fitness (reviewed in Heino and Kaitala, 1999;

Kozłowski, 1992). Researchers often describe energy balance in ectotherms as the relationship between an animal's body mass and its body size, generally referred to as body condition. If an animal is heavier than expected for its body size, it is said to be in high body condition with higher-than-average internal energy stores. Because a primary function of glucocorticoids is to mobilize energy stores, animals with low body condition often have higher circulating glucocorticoids and vice versa (Moore and Jessop, 2003; Romero, 2002; Sockman and Schwabl, 2001). For example, body condition and baseline glucocorticoids are negatively correlated during the mating season in red-spotted garter snakes, Thamnophis sirtalis concinnus (Moore et al., 2000b). In black-legged kittiwakes (Rissa tridactyla) that breed near the Arctic Circle, body condition is inversely related to both baseline and stress-induced glucocorticoid levels (Kitaysky et al., 1999). However, in a northern population of male red-sided garter snakes ( $T$. sirtalis parietalis), there is no relationship between body condition and baseline glucocorticoid concentrations (Cease et al., 2007; Moore et al., 2000a). These data suggest that the relationship between body condition and glucocorticoid levels is also context dependent.

One way to investigate differences in HPA axis sensitivity is to experimentally challenge the HPA axis. Capture stress procedures have been used extensively in the field 
and laboratory to stimulate a physiological increase in glucocorticoids (Astheimer et al., 1995; Cartledge and Jones, 2007; Coddington and Cree, 1995; Harper and Austad, 2001; Lutterschmidt and Mason, 2010; Mahmoud et al., 1989; Manire et al., 2007; Nilsson et al., 2008; Reeder et al., 2004; Ricciardella et al., 2010; Van Hout et al., 2010). Many studies have shown that responses to capture stress vary with life history characteristics (Cartledge and Jones, 2007; Lutterschmidt and Mason, 2005; Lutterschmidt et al., 2009; Reeder et al., 2004; Woodley and Moore, 2002). In populations with limited breeding opportunities, the effects of stress on hormones and behavior are often uncoupled (reviewed in Wingfield and Sapolsky, 2003). For example, Moore et al. (2000a) demonstrated that $4 \mathrm{~h}$ of capture stress during the spring mating season in red-sided garter snakes ( $T$. sirtalis parietalis) significantly elevated plasma corticosterone but did not inhibit male courtship behavior. When exogenous CORT was administered, male courtship behavior was suppressed in a threshold-dependent manner, indicating that redsided garter snakes are not completely unresponsive to elevated glucocorticoids during the mating season (Moore and Mason, 2001).

In contrast to male vertebrates, very little is known about stress responsiveness in females or whether body condition alters hormonal and behavioral responses to stress and exogenous CORT. HPA axis sensitivity varies with female reproductive state in at least some species (Cartledge and Jones, 2007; Lutterschmidt et al., 2009; Taylor et al., 2004; Vitousek et al., 2010), but whether this variation reflects changes in body condition is unknown. Because the energetic costs of reproduction are generally higher in females compared to males, they are a valuable model for understanding the interactions between 
body condition, stress responsiveness and reproduction.

We used a population of female red-sided garter snakes (T. sirtalis parietalis) in Manitoba, Canada to better understand how energy balance influences responses to capture stress. This population of snakes undergoes eight months of winter dormancy in underground hibernacula followed by a brief, intense mating season in late April-May when robust mating behavior is observed. Females ovulate, become pregnant and give birth during the summer. In the fall, snakes return to their hibernacula to begin winter dormancy again. Neither sex feeds during winter dormancy, and females feed less frequently or not at all when gravid (Charland and Gregory, 1995; Gregory et al., 1999). In these capital breeders, which rely on stored energy to fuel reproduction, the extraordinary energy demands placed on females during reproduction means that they are likely unable to recover sufficient body condition to successfully reproduce in consecutive years (Drent and Daan, 1980; reviewed in Bonnet et al., 1998;) . Thus, female red-sided garter snakes are biennial breeders that give birth approximately every other year (Garstka et al., 1982; Gregory, 1977). In a given population, approximately $50 \%$ of females will have given birth more recently and have lower body condition. Female garter snakes can therefore be collected in varying body conditions during the spring mating season. Females in higher body condition at emergence are significantly more likely to become vitellogenic, so body condition can be used as a proxy for recent reproductive history (Whittier and Crews, 1990). In the work presented here, we determined if body condition interacts with hormonal and behavioral responses to capture stress in female red-sided garter snakes. We also asked if the effects of exogenous CORT 
on female mating behavior vary with body condition.

\section{METHODS}

Experiments were conducted in the field with free-ranging red-sided garter snakes $(T$. sirtalis parietalis) at a den site located in Inwood, Manitoba, Canada during May 2004 and May 2012. The experiments described here were approved by the Manitoba Department of Conservation and Portland State University's Institutional Animal Care and Use Committee. Snout-vent length and body mass were recorded for all subjects; snakes were released at the site of capture at the completion of each study. For each experiment, we regressed body mass on snout-vent length and designated snakes with positive residuals as being in positive body condition and those with negative residuals as being in negative body condition (Cease et al., 2007; Moore et al., 2001). Final sample sizes for body condition category within each experiment are listed in the figure legends.

\section{Experimental design}

Experiment 1a: Hormonal responses to capture stress

Newly-emerged, unmated female snakes $(n=23)$ were collected directly from the den site or from newly formed mating balls between 09:30-15:00 h on 13-14 May 2012. Females were examined to confirm the lack of a mating plug. Immediately upon capture, a blood sample (approximately $200 \mu \mathrm{L}$ ) was obtained from the caudal vein of each snake to determine baseline $(0 \mathrm{~h})$ CORT and estradiol concentrations. Snakes were then isolated individually in small opaque cloth bags (approximately $20 \mathrm{~cm}$ x $20 \mathrm{~cm}$ ) for 4 hours as 
previously described (Lutterschmidt and Mason, 2005, 2010; Moore et al., 2000a, 2001). Additional blood samples were collected during ( $2 \mathrm{~h})$ and after $(4 \mathrm{~h})$ capture stress treatment.

\section{Experiment 1b: Behavioral responses to capture stress}

Immediately following the 4-h capture stress treatment, we conducted behavioral trials to determine the effects of capture stress on mating behavior $(n=19)$. For these trials, an additional 29 unmated control females were collected from the den and mating behavior was tested immediately. Females were introduced individually into arenas containing 20 males to simulate natural mating conditions (Joy and Crews, 1985).

Behavior trials for each female were monitored continuously and latency to copulate was recorded for up to 60 minutes. While attractivity was not specifically quantified in this study, all females were sufficiently attractive for mating to occur because males actively courted all females in the behavior arenas. Further, we did not observe any overt differences in activity levels or locomotory behavior between receptive and unreceptive females during the mating trials. If a female failed to mate within the 60 minute trial, we confirmed the absence of a mating plug and recorded its latency to copulate as $>60 \mathrm{~min}$. All behavior trials were conducted between 10:00-15:30 on 13-14 May 2012.

\section{Experiment 2: Behavioral responses to exogenous CORT treatment}

Unmated female snakes $(n=95)$ were collected from the den site 8-9 May 2004 and group-housed in outdoor arenas with hide-boxes and water ad libitum until the day of behavior testing (15-18 May 2004). The outdoor arenas used to house females do not 
induce a significant hormonal stress response in snakes (Cease et al., 2007; Lutterschmidt and Mason, 2005, 2010; Moore and Mason, 2001). Snakes were randomly assigned to one of three treatment groups: vehicle $(n=31)$, low CORT $(n=32)$, or high CORT $(n=$ 32). Corticosterone (Sigma, St. Louis, MO) was first dissolved in ethanol and then diluted in reptilian Ringer's solution (Lutterschmidt and Mason, 2010; Lutterschmidt et al., 2004; Moore and Mason, 2001). For the low and high CORT doses, snakes received an intraperitoneal injection of either 15 or $60 \mu \mathrm{g}$ CORT in $300 \mu \mathrm{L}$ of $5 \%$ ethanol in reptilian Ringer's, respectively. Vehicle-treated snakes received an intraperitoneal injection of 5\% ethanol in reptilian Ringer's of equal volume. Snakes were housed in arenas for 30 minutes to allow the treatments to be absorbed. Females were then introduced individually to arenas containing 20 males and latency to copulate was recorded for up to 60 minutes as described previously. All behavior trials occurred between 10:00-14:30 h on 15-18 May 2004.

\section{Blood Sampling and Radioimmunoassay}

All blood samples were collected from the caudal vein of each snake as quickly as possible (mean \pm sem: $73.1 \mathrm{~s} \pm 4.9 \mathrm{~s}$ ) using heparinized 25 -gauge needles and $1 \mathrm{cc}$ syringes. Blood samples were kept chilled $\left(1^{\circ} \mathrm{C}\right)$ after collection, and then centrifuged at the field station to isolate plasma. Plasma was transferred into fresh tubes and stored at $4^{\circ} \mathrm{C}$ before being transported on dry ice to Portland State University, where it was stored at $-80^{\circ} \mathrm{C}$ until analysis. Plasma samples were assayed for CORT and estradiol concentrations by direct radioimmunoassay following the methods described by 
Lutterschmidt and Mason (Lutterschmidt and Mason, 2005, 2009). Briefly, steroid hormones were extracted from $60 \mu$ l of each plasma sample with anhydrous ethyl ether. The solvent phase was removed and dried under nitrogen gas in a $37^{\circ} \mathrm{C}$ bead bath. Hormone extracts were then reconstituted in phosphate-buffered saline. Samples were assayed in duplicate and were incubated with approximately 12,000 c.p.m 1,2,5,7$\left[{ }^{3} \mathrm{H}\right]$ corticosterone or 2,4,6,7-[ $\left.{ }^{3} \mathrm{H}\right]$ estradiol (Product numbers: NET399250UC and NET317250UC, respectively; Perkin Elmer, Piscataway, NJ, USA) and 100 $\mu$ L antiserum at $4{ }^{\circ} \mathrm{C}$ for $18-24 \mathrm{~h}$ (CORT antibody 07120016 from MP Biomedicals, LLC, Solon, OH, USA; estradiol antibody 20R-ER012w from Fitzgerald Industries International, Acton, MA, USA). Unbound steroid was separated from bound hormone using dextran-coated charcoal and radioactivity was quantified in a liquid scintillation counter (Beckman Model No. 6500). All samples were run in a single assay with an intra-assay coefficient of variation of $14.1 \%$ for CORT and $16.9 \%$ for estradiol. Percent recovery was $69.9 \%$ and measurements were corrected for individual recovery variation.

\section{Statistical analyses}

All CORT hormone data satisfied the assumptions for parametric analysis (i.e., normal distribution and equal variance). Most estradiol samples (58 of 87, 67\%) were below the limit of detection $(1.35 \mathrm{pg} / \mathrm{ml})$, and we therefore did not perform statistical analyses on these data. In each of the behavior experiments, we rank-transformed the latency to copulate data for analysis to eliminate concerns about bounded distributions. We used SigmaPlot 12.0 (Systat Software 2010, Systat Systems, Inc., Point Richmond, 
CA, USA) for statistical analyses. All statistical comparisons were considered significant at $P \leq 0.05$.

In Experiment 1a, we investigated the influence of capture stress on plasma hormone concentrations using a two-way repeated measures ANOVA with body condition as the between-subjects factor and sampling time as the within-subjects factor followed by a Student-Newman-Keuls multiple comparisons test. To further examine the possibility that body condition influences hormonal responses to capture stress, we performed a regression of plasma CORT on body condition for each sampling time. We compared the slopes of the regression lines with an ANOVA.

In Experiment $1 b$, some females $(n=21$ of 48$)$ did not mate within the 60 -min trial, so we ranked latency to copulate to eliminate concerns about bounded distributions. Ranking necessitated that we use the non-parametric Scheirer-Ray-Hare extension of a Kruskal-Wallis ANOVA with treatment and body condition as factors. Significant main effects were further examined using a non-parametric multiple comparisons procedure (described in (Lutterschmidt and Mason, 2008; Lutterschmidt et al., 2009; Zar, 1999). Because capture stress tended to increase latency to copulate (but not significantly) we collapsed the data by treatment and reanalyzed these data using a Mann-Whitney rank sum test to better determine the influence of body condition on mating behavior.

Similarly, nearly $50 \%$ of females $(n=47$ of 95$)$ did not mate within 60 minutes in Experiment 2, so we ranked latency to copulate to eliminate concerns about bounded distributions. We examined the effect of exogenous CORT using the Scheirer-Ray Hare extension of a Kruskall-Wallis ANOVA with treatment and body condition as factors. To 
further investigate the significant differences between treatment groups, we performed a non-parametric multiple comparisons procedure as described by (Lutterschmidt and Mason, 2008, 2009).

\section{RESULTS}

Experiment 1a: Hormonal Responses to Capture Stress

Overall, there was a significant main effect of sampling time but not body condition on plasma CORT (sampling time: $F_{2,68}=3.86, P=0.03$; body condition: $F_{1,68}=1.43, P=$ 0.25, from a two-way repeated-measures ANOVA; Fig. 4.1). There was no significant interaction between factors $\left(F_{2,68}=1.57, P=0.21\right.$, from a two-way repeated-measures ANOVA). From a multiple comparisons test, baseline CORT concentrations did not differ between females with negative or positive body condition. Although the main effects of body condition were not significant, the results of the Student-Newman-Keuls multiple comparisons procedure revealed that within snakes having negative body condition, plasma CORT increased after two hours of capture stress but returned to baseline concentrations by four hours (Fig. 4.1). In contrast, plasma CORT did not increase significantly at any sampling time in snakes with positive body condition. As expected, plasma estradiol concentrations were low or undetectable at all sampling times (data not shown).

To further examine the possibility that body condition influences hormonal responses to capture stress, we performed a regression of plasma CORT on body condition at each sampling time. Corticosterone was not significantly correlated with body condition at any 
time point ( $0 \mathrm{~h}: r^{2}=0.02, P=0.53 ; 2 \mathrm{~h}: r^{2}=0.07, P=0.22 ; 4 \mathrm{~h}: r^{2}=0.003, P=0.80$,

from linear regressions; Fig. 4.2). The slopes of the $0 \mathrm{~h}$ and $2 \mathrm{~h}$ regression lines were not significantly different from each other $\left(F_{1,45}=0.47, P=0.50\right.$ from an ANOVA).

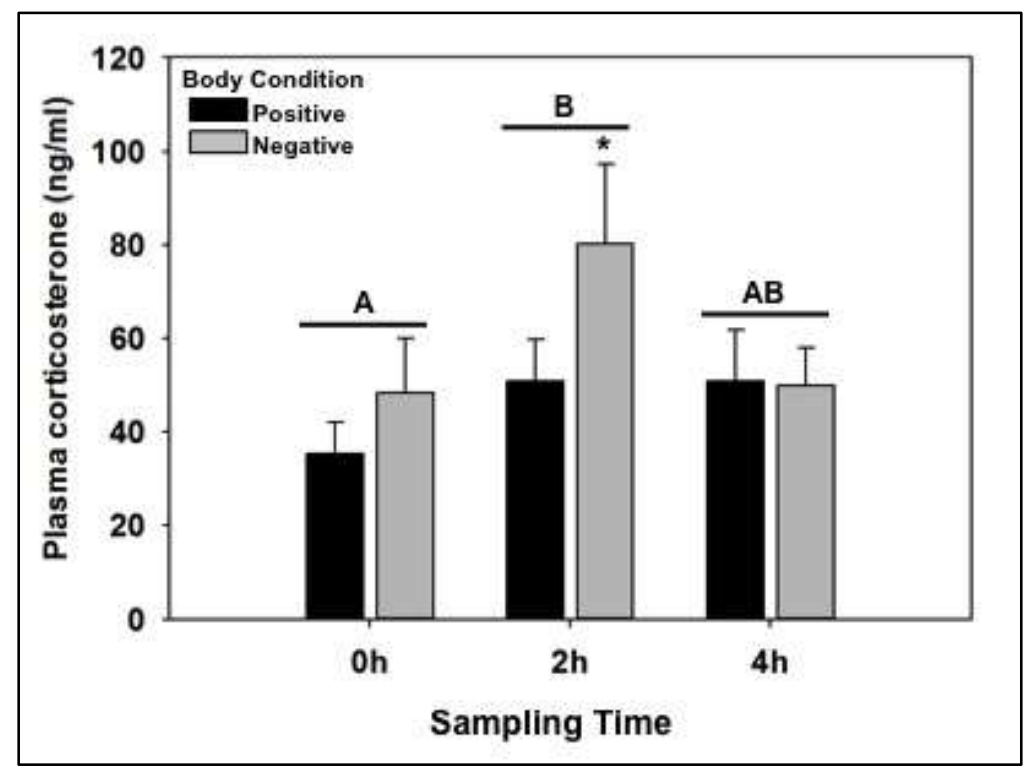

Figure 4.1. Effect of capture stress on mean plasma corticosterone (+ sem) of female red-sided garter snakes $(T$. sirtalis parietalis) before $(0 \mathrm{~h})$, during $(2 \mathrm{~h})$ and after $(4 \mathrm{~h})$ capture-stress treatment. Females are grouped by negative $(\mathrm{n}=13)$ and positive $(\mathrm{n}=10)$ body condition. Letters above the horizontal lines indicate significant differences among treatment groups. Within the $2 \mathrm{~h}$ sampling time, the asterisk indicates a significant difference between females in negative and positive body condition. All statistics are from a two-way repeated measures ANOVA followed by a Student-Newman-Keuls multiple comparisons procedure.

Experiment 1b: Behavioral Responses to Capture Stress

We confirmed that body condition did not differ between the non-stress and stresstreated snakes using a Mann-Whitney Rank Sum test $(U=190.00$, d.f. $=1, P=0.07$; data not shown). Both capture-stress treatment and body condition tended to increase latency to copulate in females, but these effects were not statistically significant (treatment: $H_{1,47}$ $=2.84, P=0.09$; body condition: $H_{1,47}=3.46, P=0.06$, from the Scheirer-Ray-Hare extension of a Kruskal-Wallis ANOVA; Fig.4.3). There was no significant interaction 
between factors $\left(H_{1,47}=0.01, P=0.92\right)$. To further examine the effects of body condition on female mating behavior, we collapsed treatment groups and reanalyzed these data using a Mann-Whitney Rank Sum test. Females with negative body condition had a significantly longer latency to copulate than females in positive body condition $(U=$ 184.00, d.f. $=1, P=0.045$; Fig 4.3).

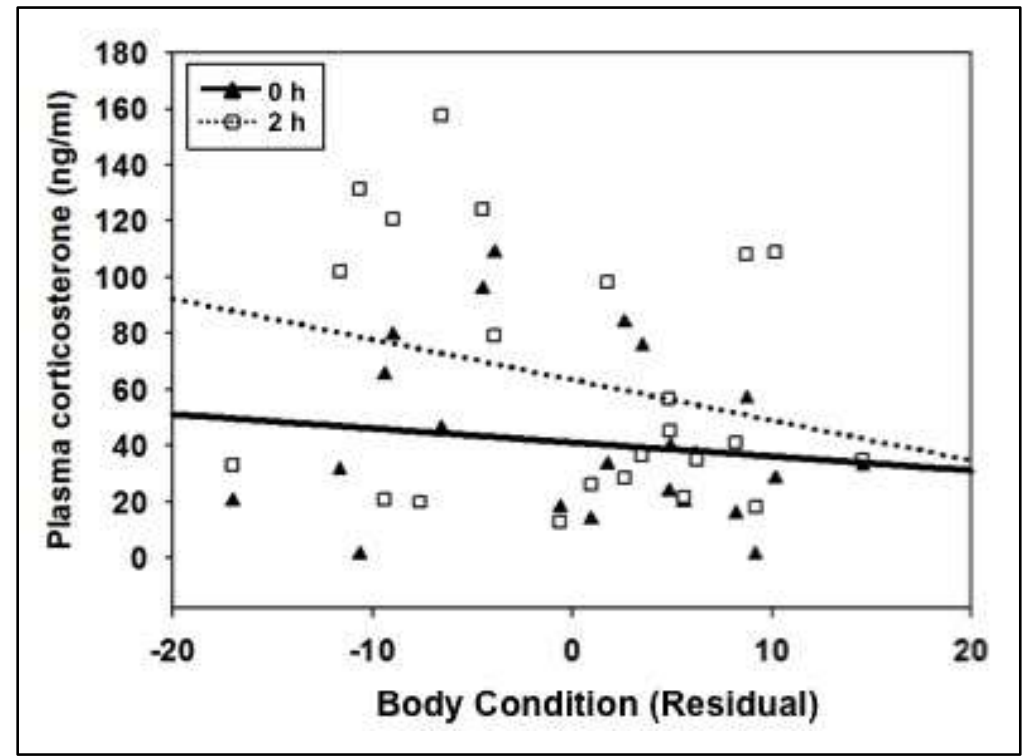

Figure 4.2. Regression of plasma corticosterone concentrations on body condition before $(0 \mathrm{~h})$ and during $(2 \mathrm{~h})$ capture stress treatment in female red-sided garter snakes, $T$. sirtalis parietalis $(\mathbf{n}=\mathbf{2 3})$. Body condition was calculated by regressing body mass on snout-vent length and designating snakes with positive and negative residuals as being in positive and negative body condition, respectively. Note that snakes in negative body condition have a larger increase in corticosterone in response to $2 \mathrm{~h}$ of capture stress compared to females in positive body condition. The slopes of these regression lines are not significantly different (from an ANOVA).

\section{Experiment 2: Behavioral Responses to Exogenous CORT}

Before examining if exogenous CORT influenced mating behavior, we first confirmed that there were no differences in body condition among treatment groups using a Kruskal-Wallis one-way ANOVA on ranks $(H=2.68, P=0.26)$. Treatment of females with the higher, $60-\mu \mathrm{g}$ dose of exogenous CORT significantly increased latency to 
copulate compared to controls $\left(H_{2,94}=8.41, P=0.02\right.$, from the Scheirer-Ray-Hare

extension of a Kruskal-Wallis ANOVA; Fig. 4.4). Negative body condition also

increased latency to copulate $\left(H_{1,94}=17.10, P<0.001\right.$; Fig. 4.4). There was no

significant interaction between factors $\left(H_{2,94}=4.42, P=0.11\right)$. Within the low CORT (15

$\mu \mathrm{g})$ treatment group, latency to copulate was increased only in females having negative

body condition (Fig. 4.4). In contrast, treatment with the high CORT dose (60 $\mu \mathrm{g})$

increased latency to copulate in females with both positive and negative body condition.

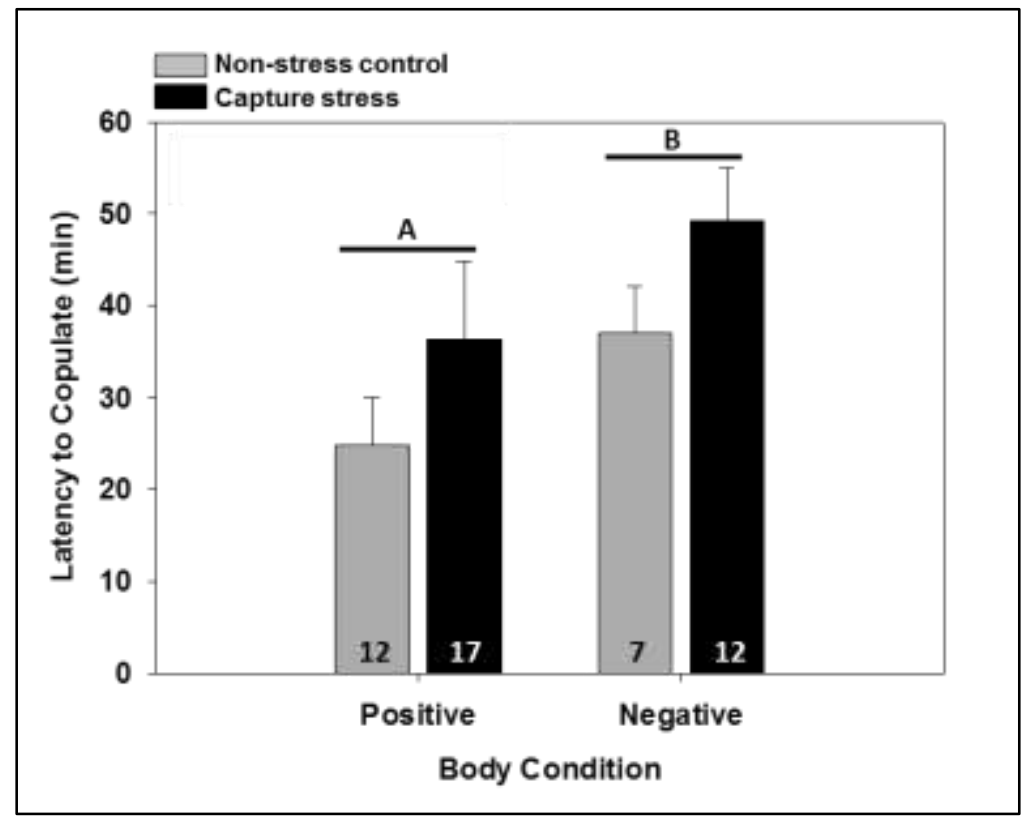

Figure 4.3. Influence of capture stress on mean latency to copulate $(+$ sem) in female red-sided garter snakes $(T$. sirtalis parietalis) with positive and negative body condition. Capture stress did not influence latency to copulate significantly $(P=0.09)$, so we collapsed the data by treatment and reanalyzed it using a MannWhitney rank sum test. The letters above the horizontal lines indicate that females with negative body condition have a significantly longer latency to copulate than females with positive body condition (from a Mann-Whitney rank sum test with treatment group collapsed).

Sample sizes are shown above the $\mathrm{x}$-axis.

\section{DISCUSSION}

We found in two different field seasons that hormonal and behavioral responses to 
capture stress and behavioral responses to exogenous CORT varied with body condition in female red-sided garter snakes (T. sirtalis parietalis). Although baseline CORT did not differ between females in negative or positive body condition, our data showed that female snakes in negative body condition were more sensitive to the effects of capture stress and exogenous CORT. For example, the observed capture stress-induced increase in CORT was driven primarily by females in negative body condition. In addition, body condition, but not capture stress, increased latency to copulate, suggesting that females were resistant to the behavioral effects of capture stress to some degree. Furthermore, only females in negative body condition increased latency to copulate in response to the low exogenous CORT dose, while all females responded to the high CORT dose. Taken together, our data suggest that variation in body condition may be related to differences in HPA axis sensitivity and/or glucocorticoid receptor density in the brain.

\section{Hormonal Responses to Capture Stress}

Capture stress procedures have been used extensively across many taxa to stimulate a physiological increase in glucocorticoids (e.g. Astheimer et al., 1995; Cartledge and Jones, 2007; Clearwater and Pankhurst, 1997; Cleary et al., 2002; Coddington and Cree, 1995; Ghisleni et al., 2012; Harper and Austad, 2001; Lutterschmidt and Mason, 2009; Mahmoud et al., 1989; Manire et al., 2007; Nilsson et al., 2008; Reeder et al., 2004; Ricciardella et al., 2010; Van Hout et al., 2010). Studies in fishes, amphibians, reptiles, birds and mammals have reported that hormonal responses to capture stress vary with season, body condition, reproductive state, and sex (Astheimer et al., 1995; Coddington 
and Cree, 1995; Cook et al., 2011; Heath and Dufty, 1998; Kitaysky et al., 1999; Moore and Jessop, 2003; Schoech et al., 1997; Taylor et al., 2004; Zerani et al., 1991). For example, Reeder et al. (2004) demonstrated that 15 minutes of restraint stress in freeranging female little brown myotis bats (Myotis lucifugus) induced higher levels of CORT in non-reproductive females as compared to reproductive females. In another example, reproductive state of female timber rattlesnakes, Crotalus horridus, (nonreproductive, reproductive or post-parturient) significantly influenced CORT responses to capture stress, but note that sample sizes were small. Because reproductive state greatly impacts female energy balance, Lutterschmidt et al. (2009) hypothesized that variation in HPA axis activity might compensate for increased energetic demands associated with pregnancy and parturition. In the current study, we further tested this hypothesis by asking if body condition influenced responses to capture stress and exogenous CORT in female red-sided garter snakes.

Moore et al. (2000a) first demonstrated that the capture stress protocol used in this study elicits a strong increase in plasma CORT in male $T$. sirtalis parietalis. Other reports in male $T$. sirtalis parietalis suggest that the magnitude of the stress response varies with baseline CORT concentrations and season (Lutterschmidt and Mason, 2005; Moore et al., 2001). Plasma CORT is generally elevated in this population during the spring mating season, and thus capture stress does not always stimulate a significant additional increase in plasma CORT above already high baseline levels (Lutterschmidt and Mason, 2005; Moore et al., 2001). During the summer and fall, capture stress increases plasma CORT more consistently, likely because baseline CORT is lower and capture stress can increase 
plasma CORT without reaching a ceiling effect. In male $T$. sirtalis parietalis, the degree to which body condition is correlated with plasma CORT concentrations appears to vary seasonally. Moore et al. (2000a) reported that in T. sirtalis parietalis, stress increased plasma CORT but the changes were not correlated with body condition during the spring mating season. In another study on seasonal variation in stress responses, baseline plasma CORT was correlated with body condition in $T$. sirtalis parietalis during every season (Moore et al., 2001).

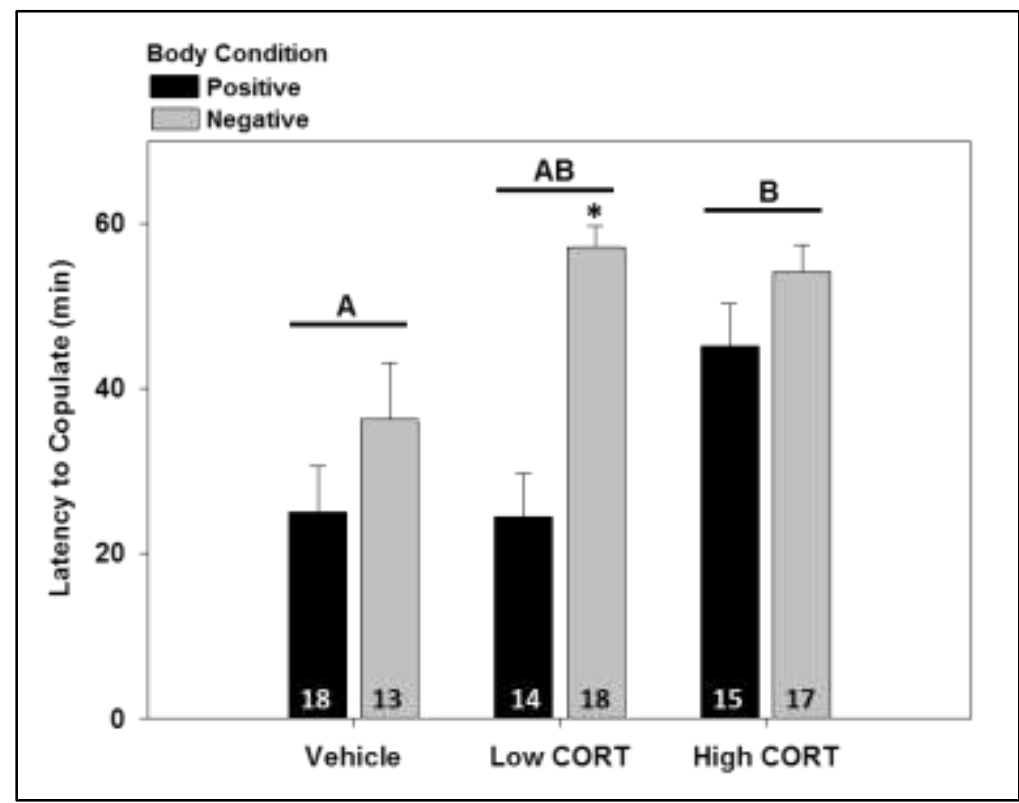

Figure 4.4. Effects of vehicle or exogenous corticosterone (CORT; low, $15 \mu \mathrm{g}$; high, $60 \mu \mathrm{g})$ on latency to copulate $(+\mathrm{sem})$ in female red-sided garter snakes ( $T$. sirtalis parietalis) with positive and negative body condition. Letters above the horizontal lines indicate significant differences among treatment groups (statistics from a Scheirer-Ray-Hare extension of a Kruskal-Wallis ANOVA). Asterisk indicates a significant difference between females with negative and positive body condition within the low CORT treatment group (from a multiple comparisons procedure). Sample sizes are shown above the $\mathrm{x}$-axis.

In the current study, plasma CORT increased significantly following $2 \mathrm{~h}$ of capture stress in female red-sided garter snakes with negative body condition (Fig. 4.1). Baseline 
CORT concentrations did not differ between females in positive or negative body condition, suggesting that the differences we observed in hormonal responses to capture stress are driven by differences in the sensitivity of the HPA axis. Similar to previous studies in males, we did not observe any correlation between body condition and baseline or stress-induced CORT in female T. sirtalis parietalis (Cease et al., 2007; Moore et al., 2000a). The apparent hormonal resistance to capture stress of females in positive body condition could be due to these females responding to capture stress more slowly or more quickly than would be detected with the time points described here $(0,2$, and $4 \mathrm{~h})$. Male T. sirtalis parietalis respond to capture stress more quickly during the summer and fall than during the spring (Moore et al., 2001). Future studies should include additional sampling times, perhaps after 30 and 60 minutes of capture stress to examine the possibility of seasonal and sex differences in the rate of response to capture stress.

To better understand if HPA axis sensitivity varies with season, sex, or body condition, experiments examining differences in both male and female responses to adrenocorticotropic hormone (ACTH) and corticotropin-releasing hormone are needed. Taken together, our current data and previously published studies suggest that external and/or internal factors modulate hormonal stress responses. One of the factors that interacts with hormonal stress responses is body condition, which may reflect differences in reproductive state (Lutterschmidt et al., 2009; Zerani et al., 1991). Thus, research on females, whose energetic costs of reproduction tend to be greater, will help us better understand the mechanistic relationship between body condition and CORT and the downstream implications for reproductive regulation. 
As expected, baseline plasma estradiol concentrations were low or undetectable and did not change in response to capture stress. Red-sided garter snakes exhibit a temporally dissociated reproductive pattern in which peak gonadal activity does not coincide with the spring mating season but rather occurs later during the summer (Crews, 1984, reviewed in Krohmer and Lutterschmidt, 2011). Thus, females mate during the spring when sex steroid hormones are relatively low, then ovulate and become pregnant during the summer coincident with increasing sex steroid levels. Plasma estradiol levels are generally very low during the spring mating season and consistent with our results, would not be expected to change markedly in response to capture stress (Lutterschmidt and Mason, 2009; Whittier et al., 1987).

\section{Behavioral Responses to Capture Stress and Corticosterone}

The physiological mechanisms that underlie the relationship between body condition and female receptivity have not been studied extensively in $T$. sirtalis parietalis. Around the time of emergence from hibernation, females experience a brief surge in plasma estradiol which appears to be important for the induction of female receptivity (Lutterschmidt and Mason, 2009; Mendonça and Crews, 1996). Our data show that body condition influences female receptivity as measured by latency to copulate, a behavioral metric of receptivity. Specifically, females in negative body condition showed an increased latency to copulate. Previous research in Galápagos marine iguanas (Amblyrhynchus cristatus) also indicates that body condition is tied to female receptivity (Vitousek et al., 2010). Receptive and non-receptive female iguanas had similar baseline 
CORT levels, but non-receptive females that were generally in lower body condition displayed a larger plasma CORT increase in response to capture stress than receptive females (Vitousek et al., 2010). In populations with limited breeding opportunities, females may remain receptive even if conditions are not optimal so that they do not miss potential mating opportunities. Our results support this possibility because females tended to respond to captures stress with increased latency to copulate, but that response did not reach statistical significance. Rather, females seem to be resistant to the effects of capture stress on mating behavior. Our results are similar to those of Moore et al. (2000a), who demonstrated that capture stress does not inhibit courtship behavior of male red-sided garter snakes during the spring mating season. These data indicate that female mating behavior in $T$. sirtalis parietalis is likely not influenced by acute stressors, but is instead strongly related to body condition.

Body condition may be an indicator of reproductive history, particularly in biennial breeders. Females that have recently given birth would likely have lower body condition and be more unreceptive because their energy reserves will have been depleted during gestation. For example, in biennially breeding Galápagos marine iguanas, glucocorticoid responses to capture stress are lower in receptive females than non-receptive females. Females in higher body condition were also more likely to be receptive (Vitousek et al., 2010). Because female $T$. sirtalis parietalis are biennial breeders, body condition may reflect primarily reproductive history. If this is the case, then we would expect to observe a bimodal distribution of body conditions in female $T$. sirtalis parietalis. We found that female body condition is distributed continuously, and therefore a more complex 
relationship between body condition and reproductive history is suggested. Additional studies focusing on the physiological mechanisms modulating receptivity in females are needed to better understand how body condition relates to reproductive history and how it influences receptivity.

Similar to previous reports in male $T$. sirtalis parietalis, we observed a threshold effect of exogenous CORT treatment on female mating behavior. The lower dose of 15 $\mu \mathrm{g}$ of CORT has been shown in previous studies to produce physiological concentrations of CORT in the plasma 2 hours post-injection (Lutterschmidt \& Mason 2010). The higher $60-\mu \mathrm{g}$ CORT treatment is a pharmacological dose that yields much higher concentrations of CORT in the blood (Lutterschmidt and Mason, 2010). In females in positive body condition, only the high dose $(60 \mu \mathrm{g})$ of CORT elicited an increase in latency to copulate, indicating that the low dose $(15 \mu \mathrm{g}$ CORT) was below the threshold to produce a behavioral effect. In contrast, females in negative body condition are more sensitive to the effects of exogenous CORT because they responded to both the high and low doses of CORT with a significant increase in latency to copulate. Our findings suggest that sensitivity to CORT differs between females in positive and negative body condition.

Future studies examining whether glucocorticoid receptor affinity and/or density in the brain varies with female body condition would provide much insight into the mechanisms underlying differences in the sensitivity to exogenous CORT. Another possible mechanism driving the differences in behavioral responses to exogenous CORT could be differences in plasma corticosteroid binding globulin (CBG) levels (Landys et al. 2006; Minni et al. 2012; reviewed in Breuner and Orchinik, 2002). Minni et al. (2012) 
demonstrated in mice (Mus musculus) that the clearance of stress-induced CORT levels, and therefore the duration of the stress response, is at least partially mediated by CBG levels in the plasma (Minni et al., 2012). In addition, body condition is positively correlated with CBG levels in male and female tufted penguins, Fratercula cirrhata (Williams et al., 2008). Taken together, these results suggest that CBG levels may mediate some of the variation we observed in female responses to exogenous glucocorticoids.

In summary, we showed that both hormonal responses to capture stress and behavioral responses to exogenous CORT varied significantly with female body condition. Our studies suggest that variation in hormonal stress responses result from differences in HPA axis sensitivity, as baseline CORT concentrations did not differ between females in negative and positive body condition. Further, we suggest that the observed differences in the sensitivity to exogenous CORT result from differences in glucocorticoid receptor density in the brain and/or CBG concentrations in the plasma. Studies addressing these possibilities would aid in understanding the mechanisms by which body condition is integrated into physiological and behavioral responses to stress and elevated glucocorticoids. Such studies examining how stress and body condition interact to regulate reproduction will help us understand how environment-driven limitations in natural populations might impact reproductive success.

\section{ACKNOWLEDGEMENTS}

We thank the Manitoba Department of Conservation and Dave Roberts for 
support in Manitoba, Canada. We thank Suzanne Estes and Ashley Maine for assistance in the field. This work was supported in part by a Gaige Fund Award from the American Society for Icthyologists and Herpetologists, a Student Research Grant from the Animal Behavior Society and a Forbes-Lea Award from Portland State University to C.A.D and Medical Research Foundation of Oregon and Portland State University Faculty Enhancement Grants to D.I.L. 


\title{
Chapter 5
}

\section{Physiological correlates of reproductive decisions: Relationships among body condition, reproductive status, and the hypothalamus-pituitary-adrenal axis in a reptile \\ In review in Hormones and Behavior}

\begin{abstract}
When opportunities to feed and reproduce are limited, females are often unable to recover sufficient energy stores to reproduce in consecutive years. Body condition has been used as a proxy for recent reproductive history in such species. We previously found that glucocorticoid responses to capture stress vary with body condition in female redsided garter snakes (Thamnophis sirtalis parietalis), a species with limited seasonal breeding and feeding opportunities. Because variation in glucocorticoid receptor (GR) protein in the brain could explain these differences, we first assessed GR protein content in females in different body conditions. To investigate if body condition during the spring mating season accurately reflects recent reproductive history, we measured glucocorticoid responses to stress in females with different body conditions, assessed their mating behavior and brought mated females to our lab to determine which females would give birth during the summer (i.e., were parturient). Female red-sided garter snakes reproduce biennially, and therefore mated females that did not give birth were assumed to have given birth the previous summer and were deemed post-parturient. In
\end{abstract}


this study, glucocorticoid stress responses and mating behavior did not vary with body condition, nor was body condition related to brain GR or reproductive condition (parturient vs post-parturient). Only unreceptive females showed a significant stressinduced increase in glucocorticoids, suggesting that reduced stress responsiveness is associated with receptivity. Parturient females mated faster (were more proceptive) than post-parturient females. These data suggest that HPA axis activity influences reproductive "decisions" by modulating receptivity, while proceptivity is related primarily to recent reproductive history.

\section{INTRODUCTION}

When opportunities for feeding and reproduction are limited, animals must make important "decisions" about whether or not to reproduce. Reproducing under unfavorable conditions jeopardizes both survival and overall reproductive success, but failure to reproduce under favorable conditions also jeopardizes fitness. The high stakes of navigating the trade-offs between reproduction and survival mean that the physiological mechanisms used by either sex to make reproductive decisions are critically important, yet they remain poorly understood in nearly all species.

The hypothalamus-pituitary-adrenal (HPA) axis receives projections from many brain regions (Whitnall, 1993) and thus is ideally positioned to integrate information about internal status, such as energy balance and reproductive history, with external cues, such as day length, temperature, and the presence of conspecifics. All of these cues potentially inform reproductive decisions. Hence, the HPA axis may integrate 
information from a variety of neural and neuroendocrine pathways and then contribute to reproductive decision-making via modulating the production of its major endocrine factor, glucocorticoids. In turn, glucocorticoids mobilize energy stores, fueling energetically intensive processes such as reproduction and migration (Landys et al., 2006; Schoech et al., 2009). As such, glucocorticoid concentrations are often elevated during the breeding season compared to other times of the year (Romero, 2002).

In addition to allostatic changes in baseline glucocorticoids, acute environmental perturbations can elicit a stress-induced increase in glucocorticoids that generally activates physiological processes and behaviors that support immediate survival while suppressing non-essential processes (Sapolsky et al., 2000). For example, activation of the HPA axis suppresses reproductive physiology and behavior in many animals (Crossin et al., 2016; Wingfield and Romero, 2001); these results can be mimicked by treatment with exogenous glucocorticoids. Taken together, these data suggest that while elevated glucocorticoids are often necessary to fuel the energetic demands of reproduction, if circulating glucocorticoid concentrations exceed some upper threshold, reproduction can be suppressed (Wingfield et al., 1998). In other words, there is a physiological range of glucocorticoid concentrations that facilitates and supports reproductive efforts.

Many studies examining the relationship between the HPA axis and reproduction hinge upon perturbing the HPA axis with standardized capture stress protocols. Such studies often report significant variation in both the magnitude of the stress-induced increase in glucocorticoids and how increased glucocorticoids affect reproduction (Wingfield and Romero, 2001; Wingfield and Sapolsky, 2003). Although there are fewer 
studies in females compared to males, the available data suggest that female reproductive condition is also an important factor. Indeed, glucocorticoid responses to capture stress vary with reproductive condition in some species of all vertebrate classes (Anderson et al., 2014; Bauer et al., 2014; Jessop, 2001; Lutterschmidt et al., 2009; Narayan and Hero, 2013; Schreck et al., 2001; Vitousek et al., 2010; Wingfield et al., 1992; Woodley and Moore, 2002). In female tree lizards (Urosaurus ornatus), capture stress treatment elicits a significant increase in glucocorticoids unless the female is gravid, in which case no increase is observed (Woodley and Moore, 2002). These results suggest that the sensitivity of the HPA axis is modulated with the changing demands of the reproductive cycle, potentially reducing any negative impacts of glucocorticoids on female reproduction and/or embryonic development (Lupien et al., 2009).

Females of live-bearing species in particular are subject to extraordinary energetic demands during reproduction, and some species cannot recover sufficient energy stores to reproduce in consecutive breeding seasons (e.g., Bonnet et al., 1998; Gregory, 2006; Whittier and Crews, 1990). A female's recent reproductive history can therefore influence whether or not she will reproduce in a given year. When this is the case, body condition is expected to vary with reproductive history such that females that have recently given birth have lower body condition compared to those that have not. In some species, the magnitude of the stress response varies with body condition (Dayger et al., 2013; Vitousek et al., 2010; Vitousek and Romero, 2013), but whether this variation is related specifically to reproductive history, rather than body condition more generally, is unknown. For example, female red-sided garter snakes (Thamnophis sirtalis parietalis) in 
below-average body condition, but not above-average body condition, increased glucocorticoids in response to capture stress during the spring mating season (Dayger et al., 2013). Females in below-average body condition were also more sensitive to lower doses of exogenous corticosterone than those in above-average body condition, suggesting that the sensitivity of the HPA axis varies with body condition in this species (Dayger et al., 2013). Considering female red-sided garter snakes are biennial breeders, and that reproductive condition and body condition are both related to variation in stress responsiveness in other species, we hypothesized that the association between body condition and stress responsiveness actually reflects variation in reproductive condition.

We tested this hypothesis in a well-studied population of red-sided garter snakes (Thamnophis sirtalis parietalis) by directly investigating the relationship between body condition, glucocorticoid and behavioral stress responses, and reproductive condition in females. Because variation in the quantity of glucocorticoid receptors (GR) could explain the relationship between stress responses and body condition, we first compared brain GR peptide content and glucocorticoid stress responses in females in different body conditions. Glucocorticoids can bind both GR and mineralocorticoid receptor (MR) (Landys et al., 2006; Whitnall, 1993), although GR is generally thought to mediate stressinduced signaling. Surprisingly, no published studies have investigated whether the quantity of GR varies with body condition in any taxa. Next, we tested whether behavioral stress responses vary with body condition using two measures of female reproductive behavior: receptivity and, among receptive females, proceptivity. Finally, we tested whether body condition, glucocorticoid stress responses and/or behavioral 
stress responses vary with reproductive history. We posit that body condition reflects recent reproductive history and thereby plays a role in the relationship between the HPA axis and reproductive decisions.

\section{Natural History of Study Organism}

We conducted these studies in a population of red-sided garter snakes in Manitoba, Canada. During the winter, red-sided garter snakes brumate communally in underground dens for approximately 8 months before emerging in late April through May, upon which they engage in a short mating period lasting about 4 weeks (Gregory, 1973). Males begin emerging first, but remain at the den site searching for females to court. Females emerge during the course of the mating season and are courted vigorously by up to 100 males in a single mating ball (Garstka et al., 1982). If receptive to mating, the female will exhibit proceptive behaviors (Beach, 1976), such as remaining stationary during male courtship, raising the tail and gaping the cloaca to permit intromission (Carpenter, 1977; Crews, 1976; Garstka et al., 1982). If the female is unreceptive, it will evade courtship by males, often moving away suddenly and quickly (Crews, 1976; Mendonça and Crews, 1996). Females disperse to the summer feeding grounds within 24 $\mathrm{h}$ of emergence, while males can remain at the den site for several weeks seeking further mating opportunities before eventually migrating to summer feeding grounds. Neither sex feeds during winter dormancy or the spring mating season (Gregory, 1973; O’Donnell et al., 2004; Whittier and Crews, 1990). Thus, energy stores acquired the previous year must sustain the snakes through the mating season and the initial migration to summer feeding 
grounds.

During the summer, females may become vitellogenic, ovulate, and fertilize eggs from sperm stored in the oviduct (Halpert et al., 1982). In late summer, pregnant females give birth to live young at the feeding grounds. Males and females migrate back to the den site in early fall before descending underground for the winter. The short, 3-month feeding season experienced by northern-latitude populations of red-sided garter snakes, coupled with the high energetic demands of viviparous reproduction, means that females are unable to reproduce in consecutive years and are therefore biennial breeders (Gregory, 2006; Whittier and Crews, 1990). Red-sided garter snakes are also capital breeders that use stored energy to fuel reproduction (Bonnet et al., 1998; Drent and Daan, 1980). Thus, during the spring mating season, females that gave birth the previous summer are expected to be in lower body condition than females that did not give birth and instead spent the summer feeding season acquiring and storing energy for future reproductive efforts.

\section{MATERIALS AND METHODS}

Experiments were conducted in the field with free-ranging red-sided garter snakes at a den site located in Inwood, Manitoba, Canada. The experiments described here were approved by Portland State University's Institutional Animal Care and Use Committee and conducted under the authority of Wildlife Scientific Permit WB14930 issued by the Manitoba Department of Sustainable Development (formerly the Department of Conservation). 


\section{Experimental design}

Based on previous reports that HPA axis sensitivity varies with body condition, we asked if GR in the brain differs with body condition. We first calculated a general body condition index by weighing and measuring 100 randomly-selected females during the 2013 spring mating season. We then regressed body mass on snout-vent length from these females and used this relationship to preferentially collect additional females with highly negative residuals $(\mathrm{n}=11$; mean \pm SEM: $-6.13 \pm 1.10)$ and highly positive residuals $(\mathrm{n}=9$; mean \pm SEM: $7.88 \pm 2.47)$ on 12 May 2013 between 11:00h and 18:00h. These methods allowed us to increase the probability of seeing a difference in GR between body condition groups, if one exists. We confirmed that females were unmated by checking for the absence of a mating plug in the cloaca. To date, no study has characterized GR peptide content in the brain of snakes of either sex and therefore we also collected a subset of males $(n=9)$ to investigate potential sex differences in GR peptide expression. Snakes were euthanized and the brains quickly dissected into left and right hemispheres and immediately frozen on dry ice. The time elapsed between collection of the snake and freezing of the brain was always less than 10 min (mean \pm SEM: 6:30 $\pm 0: 14)$. The brains were transferred to liquid nitrogen upon return to the research station and transported to Portland State University on dry ice, where we performed Western blot analysis for GR peptide.

In a separate experiment to assess the relationship between body condition, stress responses and reproduction, we collected 80 female snakes during the spring mating season from 5-8 May 2013. Females were captured from mating balls and confirmed to 
be unmated by checking for the absence of a copulatory plug in the cloaca. Blood samples $(150 \mu \mathrm{L})$ were collected immediately upon capture from the caudal vein (mean sampling time \pm SEM: $78.6 \pm 5.9 \mathrm{~s})$. All snakes were then subjected individually to $4 \mathrm{~h}$ of capture stress in small, opaque cloth bags (approximately $20 \mathrm{~cm}$ x $20 \mathrm{~cm}$ ). Additional blood samples were collected during ( 1 and $2 \mathrm{~h}$ ) and after $(4 \mathrm{~h})$ capture stress.

Immediately after capture stress treatments each female was removed from the stress bag and introduced individually into a behavior arena ( $1 \mathrm{~m}$ x $1 \mathrm{~m}$ x $1 \mathrm{~m}$ constructed of nylon cloth) containing 15 male snakes. We monitored female behavior continuously for up to 60 minutes and recorded two measures of female reproductive behavior during the trials: receptivity and proceptivity (Beach, 1976). Receptivity was determined by recording whether or not a female mated, similar to Vitousek et al. (2010). In those females that were receptive, we assessed proceptivity by measuring each female's latency to copulate. Because female red-sided garter snakes must mate cooperatively, females with shorter latencies to copulate are more motivated to mate, or more proceptive.

To reduce the possibility that a female's unreceptive behavior was related to the capture stress treatment, females that did not mate during the behavior trial were given additional opportunities to mate with newly-collected males on successive days. In this way, we could more clearly designate females as either receptive or unreceptive. Of the 80 females subjected to capture stress, 30 mated during the behavior trial (designated as receptive), 17 mated on a successive day, and 33 never mated despite repeated opportunities (designated as unreceptive). To more clearly compare the stress responsiveness of unreceptive and receptive females, we excluded females that mated on 
a successive day because these females represent a group with an intermediate phenotype.

Following the behavior trial, individual snakes were scale-clipped on the ventral surface with a unique identifying number. We recorded snout-vent length and body mass of each snake and used these data to calculate body condition index. We performed a regression of natural log-transformed body mass on natural log-transformed snout-vent length and designated females with residuals $<0$ as having a negative body condition index and females with residuals $>0$ as having a positive body condition index (Davis et al., 2012; Dayger et al., 2013; Dayger and Lutterschmidt, 2016; Jessop et al., 2004, 2003; Moore et al., 2000b).

No reliable method exists for determining if a female garter snake captured during the spring gave birth during the immediately preceding summer (i.e., its reproductive history). Thus, we returned the mated females to our laboratory and recorded which of them gave birth during the summer to assess reproductive history. To more reliably determine the relationship between reproductive behavior, reproductive history and the sensitivity of the HPA axis, we limited our comparisons to the receptive females that mated during the behavior trial $(\mathrm{n}=30)$. We released the 33 unreceptive females and the 17 females that mated on a subsequent day at the site of capture. Mated females were held in an outdoor arena (1 m x $1 \mathrm{~m} \times 1 \mathrm{~m})$ with access to a hide box and fresh water $a d$ libitum until being transported to Portland State University in late May 2013 to assess reproductive history.

At Portland State University, snakes were housed in environmental chambers 
under summer-like environmental conditions (16 h: $8 \mathrm{~h}$ L:D photoperiod, $24^{\circ} \mathrm{C}: 18^{\circ} \mathrm{C}$ thermoperiod). Snakes were housed individually in 40-1 aquaria and were fed twice weekly with vitamin-fortified fish and earthworms. On 22 August 2013, the environmental conditions were adjusted to late summer-like conditions (12 h: $12 \mathrm{~h} \mathrm{L:D}$ photoperiod, $18^{\circ} \mathrm{C}: 10^{\circ} \mathrm{C}$ thermoperiod). At that time, females that were confirmed to be pregnant by palpation were monitored twice daily until parturition occurred. Within 24 hours of parturition, each litter was removed from the mother's cage and housed in a separate 40-1 aquarium. Neonates were fed every other day with vitamin-fortified chopped fish and minced earthworms. Water was provided ad libitum to all snakes. Because female red-sided garter snakes are biennial breeders (Gregory, 2006; Whittier and Crews, 1990), females that gave birth in the lab during the summer $(n=11)$ were designated as parturient (i.e., going to give birth) when we captured them during the spring. Females that did not give birth $(n=19)$ were designated post-parturient (i.e., presumed to have given birth the previous summer).

\section{Western blot}

We performed a Western blot analysis on GR peptide in the brains of male and female snakes similar to the methods of Vijayan et al. (2003). The right hemisphere from each snake was thawed on ice and homogenized in $2 \mathrm{~mL}$ of homogenization buffer $(0.2 \%$ SDS, $32 \mathrm{mM}$ Tris) per gram of tissue using a disposable pellet pestle. The brain homogenate was incubated at $100^{\circ} \mathrm{C}$ for $15 \mathrm{~min}$ and centrifuged at $12,000 \mathrm{x} \mathrm{g}$ for $10 \mathrm{~min}$ at room temperature. The supernatant was collected and its protein concentration 
quantified using a standard Bradford assay (Bradford, 1976). We used recombinant GR (rGR) peptide (Product no. RP-510A, ThermoFisher Scientific, LLC, Waltham, MA, USA) as a positive control.

For Western blots, $5 \mu \mathrm{g}$ of total protein from each sample or $0.1 \mu \mathrm{g}$ rGR was separated by electrophoresis on $10 \%$ SDS-polyacrylamide gels. Samples were randomly assigned to one of 4 gels with a control lane of rGR in each gel. After separation, proteins were transferred to Immobilon-P PVDF membranes (Product no. IPVH00010, EMD Millipore, Darmstadt, Germany) by electroblotting at $30 \mathrm{mAmps}$ for $16 \mathrm{~h}$ at $4^{\circ} \mathrm{C}$. Effective transfer and equal protein loading across all samples were confirmed similar to Pennington et al. (2008) with MemCode reversible protein staining kit (Product no. 24580, Pierce Biotechnology, Rockford, IL, USA). Blots were rinsed for $5 \mathrm{~min}$ in nanopure $\mathrm{H}_{2} \mathrm{O}$ and then blocked for $2 \mathrm{~h}$ in $1 \%$ blotto [5\% dry milk and $1 \%$ bovine serum albumin in Tris-buffered saline containing $0.1 \%$ Tween-20 (TBST)] at $25^{\circ} \mathrm{C}$ with gentle agitation. Blots were then incubated for $16 \mathrm{~h}$ in a 1:1000 dilution of polyclonal rabbit anti-GR antibody (Product no: ab3578, Abcam, Cambridge, UK) in $2 \%$ bovine serum albumin in TBST at $4^{\circ} \mathrm{C}$ with gentle agitation. This antibody was generated against a synthetic peptide corresponding to amino acids 346-367

(DQKPIFNVIPPIPVGSENWNRC) of human GR. Blots were washed 3 x 5 min each in TBST. Following washes, blots were incubated for $5 \mathrm{~h}$ in a 1:5000 dilution of horseradish peroxidase-labeled anti-rabbit secondary antibody (Product no., 111-035-003, Jackson ImmunoResearch Laboratories, Inc, West Grove, PA, USA) in 2\% bovine serum albumin in TBST at $25^{\circ} \mathrm{C}$ with gentle agitation. Blots were washed $3 \times 5$ min in TBST and 
exposed to SuperSignal West Pico chemiluminescent reagent (Product no. 34079, Pierce Biotechnology). Blots were enclosed in a transparent document sleeve and exposed to Xray film (CL-Xposure Film, Product no. 34090, ThermoFisher Scientific). Densitometry was conducted on blots using ImageJ software (U.S. National Institutes of Health, Bethesda, MD, USA). Any sample that did not result in a distinct $72 \mathrm{kDa}$ band that was reliably quantifiable by densitometry was not included in the final analyses $(n=5$ of 29 samples). Due to limited protein concentrations, these samples could not be reanalyzed. Final sample sizes were therefore 9 females in negative body condition, 7 females in positive body condition and 8 males. To confirm that the anti-GR antibody we used is specific for GR protein in snake tissues, we conducted a series of control tests, including omission of the primary and secondary antibodies and preabsorption tests. For the latter, we incubated the GR antibody with $6.25 \mu \mathrm{g}$ synthetic GR peptide (Product no. ab5019, Abcam, Cambridge, UK) per ml of antibody solution for $12 \mathrm{~h}$ prior to performing the Western blot as described.

\section{Blood sampling and radioimmunoassay}

Blood samples were collected using heparinized 25-g needles and 1-cc syringes. Samples were stored on ice until return to the field station, where they were centrifuged to separate plasma from blood cells. Plasma was stored frozen at $-4^{\circ} \mathrm{C}$ before being transported on dry ice to Portland State University and stored at $-80^{\circ} \mathrm{C}$ until analysis. Plasma was analyzed for corticosterone concentrations using direct radioimmunoassay methods previously validated by Lutterschmidt and Mason (2009, 2005). Briefly, steroid 
hormones were extracted from $5-10 \mu \mathrm{L}$ of plasma with anhydrous ethyl ether. The solvent phase was then evaporated under nitrogen gas in a $37^{\circ} \mathrm{C}$ bead bath and the extracted hormones were reconstituted with phosphate buffered saline. Samples were assayed in duplicate by incubating each replicate with approximately $12,000 \mathrm{cpm}$ of 1,2,6,7 - $\left[{ }^{3} \mathrm{H}\right]-$ corticosterone (Product number: NET317250UC, Perkin Elmer, Piscataway, NJ, USA) and $100 \mu \mathrm{L}$ antibody (Product number: 07120016, MP Biomedicals, LLC, Solon, OH, USA) at $4^{\circ} \mathrm{C}$ for $16-20 \mathrm{~h}$. Unbound hormone was removed from bound hormone using dextran-coated charcoal and the radioactivity of each bound fraction was quantified in a liquid scintillation counter (Beckman 6500, Beckman Coulter, Inc., Brea, CA, USA). The mean intra-assay coefficient of variation was $18.7 \%$ and the inter-assay coefficient of variation was $19.4 \%$. Concentrations were corrected for individual recovery variation; the mean percent recovery was $76.3 \%$. The mean limit of detectability was $19.6 \mathrm{pg} / \mathrm{mL}$.

\section{Statistical analyses}

We used SigmaPlot 12.0 (Systat Software 2010, Systat systems, Inc., Port Richmond, CA, USA) to perform all statistical analyses. Statistical comparisons were considered significant at $p \leq 0.05$. Except where noted, all data met the assumptions of normality and equal variance required for parametric tests. Where necessary, we transformed the data to meet the parametric assumptions. For two of the multifactor repeated measures analyses of variance (ANOVA), the data could not be transformed to meet the assumption of normality, and nonparametric options for multifactor mixed 
model ANOVA do not exist. ANOVAs are robust against modest deviations from normality (Sheskin, 2007; Zar, 1999), and therefore we proceeded with parametric twoway repeated measures ANOVAs in these instances as in Lutterschmidt and Mason (2009, 2008).

Because the magnitude and duration of the stress response is associated with the number of GRs in the brain (Ronchi et al., 1998), we first compared the quantity of GR peptide in the brain of females in different body conditions using a Mann-Whitney U-test. We also investigated if the amount of GR peptide varies with sex using a Mann-Whitney U-test.

We used a t-test to determine if body condition differed between receptive and unreceptive females. We then examined if corticosterone responses to capture stress varied with body condition using a two-way repeated measures ANOVA on corticosterone concentrations with sampling time as the within-subjects factor and body condition category as the between-subjects factor; data from receptive and unreceptive females were combined for analysis. The corticosterone data for this test could not be transformed to meet the assumption of normality, but the untransformed data most closely approached normality based on a Shapiro-Wilk test. Therefore, we proceeded with the ANOVA using the untransformed data. We further examined the significant main effects using a Student-Newman-Keuls multiple comparisons procedure.

To determine if corticosterone responses to capture stress varied with receptivity, we used a two-way repeated measures ANOVA with sampling time as the withinsubjects factor and receptivity status (i.e., receptive or unreceptive) as the between- 
subjects factor. We further investigated the significant main effects using a StudentNewman-Keuls multiple comparisons test. Within receptive females, we used a t-test to investigate whether female proceptivity varies with body condition index. Female proceptivity was assessed as the latency to copulate in receptive females. To examine if body condition upon spring emergence accurately reflects reproductive history, we compared the body condition index of parturient and post-parturient females using a ttest. We then examined if the corticosterone response to capture stress varies with reproductive history using a two-way repeated measures ANOVA with sampling time as the within-subjects factor and reproductive condition (i.e., parturient or post-parturient) as the between-subjects factor. The corticosterone data for this test could not be transformed to meet the assumption of normality, but the untransformed data most closely approached normality based on a Shapiro-Wilk test. Therefore, we proceeded with the ANOVA using the untransformed data. Lastly, we investigated if proceptivity was related to reproductive condition by comparing the latency to copulate between parturient and post-parturient females using a t-test.

\section{RESULTS}

The quantity of GR peptide in the brain may influence the magnitude and duration of the stress response, and thus we asked if the expression of GR peptide varied with body condition. We observed two bands in our Western blots at $\sim 72 \mathrm{kDa}$ and $\sim 55 \mathrm{kDa}$. Our results are similar to those of other studies reporting variation in the size of the GRspecific band (Arora et al., 2013), with two protein bands observed in pig brain (Weaver 
et al., 2000), mouse brain (Spencer et al., 2000) and mouse tumors (Arora et al., 2013). Multiple isoforms of GR mRNA of differing nucleotide lengths have also been observed in humans in heart, placenta, lung, liver, skeletal muscle, and pancreas (Lu and Cidlowski, 2006). Together, these data support the observed size variation in GR protein in this study. Our control tests using GR antiserum preabsorbed with synthetic GR peptide, as well as omission of the primary and secondary antibodies, completely abolished staining of both the $\sim 72 \mathrm{kDa}$ and $\sim 55 \mathrm{kDa}$ bands in Western blot. Moreover, we identified the cDNA for GR in garter snake brain using PCR with degenerate primers developed from consensus sequences. Similar to Oka et al. (2013), we found high sequence similarity; the percent identity of snake GR with that of human GR (Accession number XM_011537637.1) is 81\% (unpublished data). Together, these results support the conclusion that the two bands we observed in Western blot are in fact GR proteins in garter snake brain.

We found no difference in the quantity of GR peptide between females in positive and negative body condition $(U=19.00$, df. $=1, p=0.20$, Fig. 5.1A-B). For comparison purposes, males $(n=8)$ had a mean \pm SE of $20.95 \pm 7.14$ optical density units (ODU); females $(n=16)$ had a mean \pm SE of $14.52 \pm 3.95$ ODU. The amount of GR peptide did not differ between males and females ( $U=56.00$, d.f. $=1, p=0.65$, data not shown $)$.

Body condition index did not differ between receptive and unreceptive females $(\mathrm{t}$ $=1.433, p=0.16$, data not shown). We therefore collapsed these groups to examine whether female corticosterone stress responses varied with body condition, as reported previously (Dayger et al., 2013). We found a significant main effect of sampling time 
$\left(F_{3,251}=7.20, p<0.001\right)$ but not body condition category $\left(F_{1,251}=0.11, p=0.74\right)$ on plasma corticosterone (Fig. 5.2). There was no significant interaction between factors $\left(F_{3,251}=0.98, p=0.40\right)$.

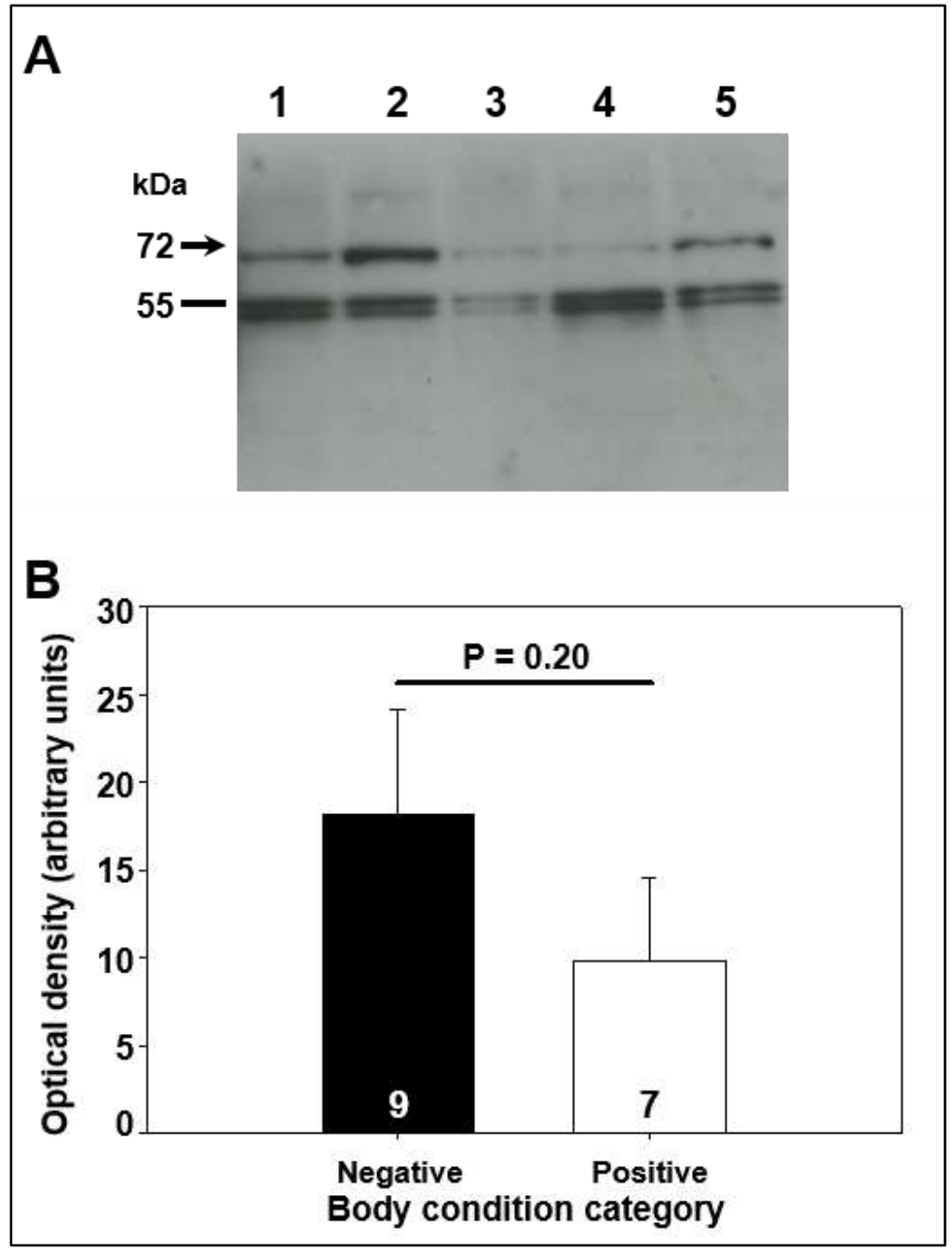

Figure 5.1. Determination of glucocorticoid receptor (GR) in the brain of red-sided garter snakes (Thamnophis sirtalis parietalis) via Western blot. (A) Representative immunoblot of snake brain; lanes are (1) negative body condition female, (2) male, (3) male, (4) positive body condition female, (5) negative body condition female. The right hemisphere of each brain was used for experimental immunoblots; males and females were randomly assigned to one of 4 gels. (B) Semi-quantitative optical density of the $\sim 72 \mathrm{kDa}$ GR band $($ mean + SEM) from female garter snake brains. Numbers along the abscissa are sample sizes in each body condition category. 
We then collapsed body condition category and compared corticosterone stress responses between receptive and unreceptive females. We found a significant main effect of sampling time $\left(F_{3,251}=7.13, p<0.001\right)$ but not receptivity category $\left(F_{1,251}=0.96, p=\right.$ 0.33) on corticosterone responses to capture stress (Fig. 5.3). However, there was a significant interaction between factors $\left(F_{3,251}=2.63, p=0.05\right)$. When we investigated the interaction further with a Student-Newman-Keuls multiple comparisons test, we found that only unreceptive females significantly responded to capture stress; receptive females were relatively resistant to capture stress and did not exhibit an increase in corticosterone (Fig. 5.3). These differences in stress responsiveness do not result from variation in body condition because body condition index did not differ significantly between receptive and unreceptive females, as noted previously.

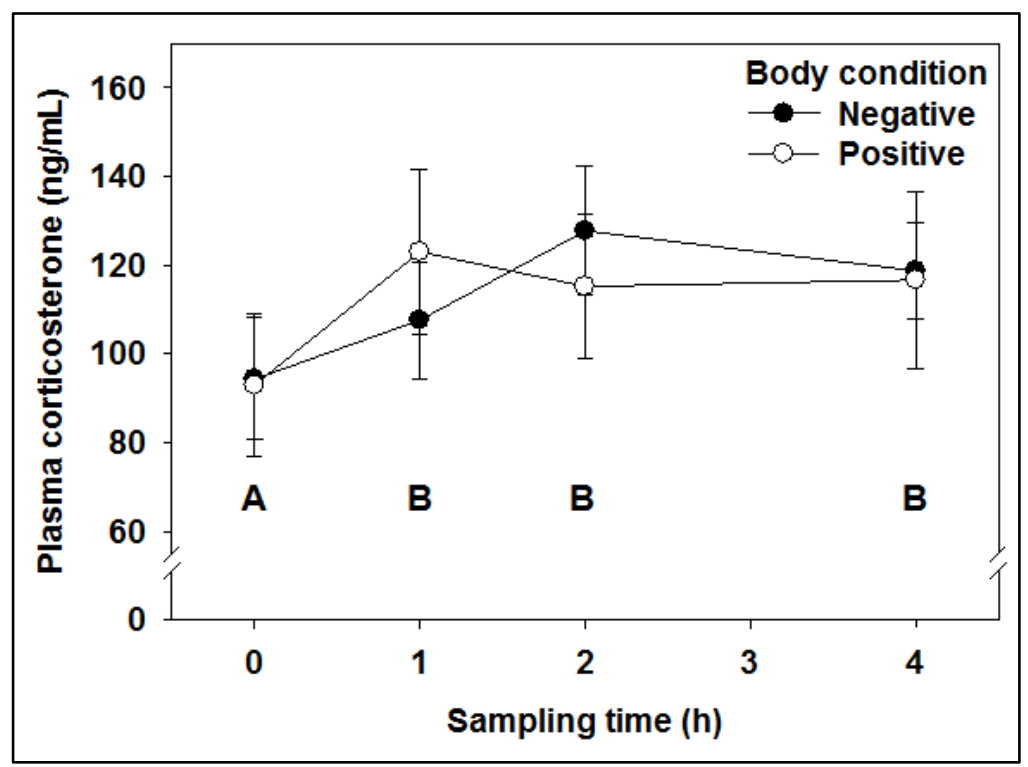

Figure 5.2. Corticosterone responses to capture stress in female red-sided garter snakes (Thamnophis sirtalis parietalis) in negative $(n=35)$ and positive $(n=28)$ body condition; receptive and unreceptive female groups were collapsed for analysis. Each data point is the mean corticosterone concentration \pm 1 SEM. Letters indicate significant differences among sampling times. 


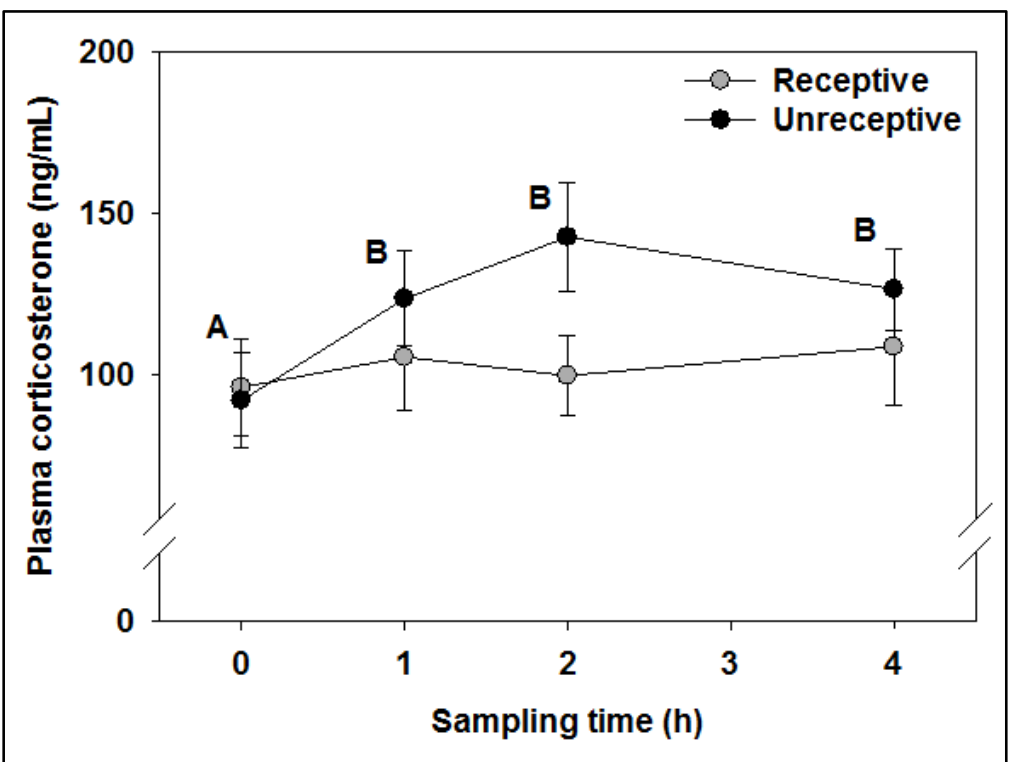

Figure 5.3. Corticosterone responses to capture stress in receptive $(n=30)$ and unreceptive $(n=33)$ female red-sided garter snakes (Thamnophis sirtalis parietalis). Each data point is the mean corticosterone concentration \pm 1 SEM. Letters indicate significant differences among sampling times within unreceptive females; corticosterone did not change significantly at any time during capture stress in females that were later receptive to mating.

Because body condition may primarily reflect reproductive status, we directly tested whether females that gave birth the preceding summer (post-parturient) were in lower body condition during the spring mating season than females that would give birth during the current summer (parturient). Although there was a trend in this direction, we found no significant difference in body condition index between parturient and postparturient females $(t=-1.08, \mathrm{df}=28, p=0.29$; Fig. 5.4A). We then examined if corticosterone stress responses differed between parturient and post-parturient females and found no significant main effects of sampling time $\left(F_{3,119}=0.11, p=0.95\right)$ or reproductive condition $\left(F_{1,119}=0.56, p=0.46\right.$; Fig. 5.4B $)$. There was no significant interaction between factors $\left(F_{3,119}=1.91, p=0.14\right)$. Finally, we examined if behavioral 


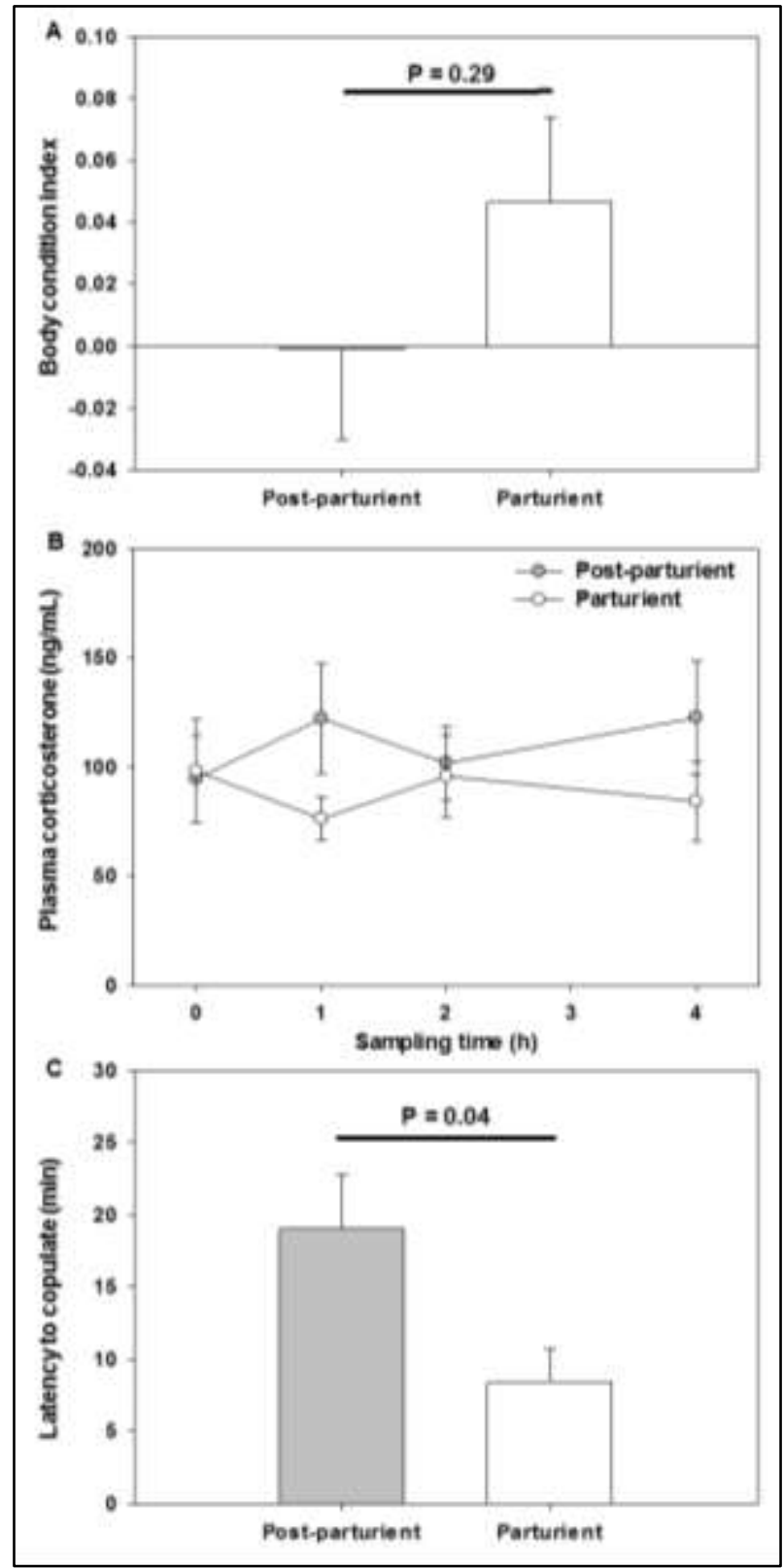

Figure 5.4. Relationship between reproductive condition and (A) body condition index at spring emergence, (B) stress-induced plasma corticosterone, and $(C)$ latency to copulate, a measure of proceptivity, in female red-sided garter snakes (Thamnophis sirtalis parietalis).

Reproductive condition was categorized as postparturient $(\mathrm{n}=19)$ and parturient $(\mathrm{n}=11)$. Female red-sided garter snakes produce young biennially, and thus females were deemed parturient if they gave birth the summer immediately following their collection and post-parturient if they did not give birth. 
stress responses varied with reproductive condition by comparing the latency to copulate between parturient and post-parturient females. We found that parturient females mated more quickly than post-parturient females following capture stress treatment $(t=2.12$, d.f. $=28, p=0.04$; Fig. 5.4C). Importantly, latency to copulate did not differ with body condition ( $p=0.66$; data not shown) in these receptive females, and therefore the differences in proceptivity reported here do not simply reflect the trending differences in body condition between parturient and post parturient females.

\section{DISCUSSION}

We posited that body condition primarily reflects recent reproductive history and is related to glucocorticoid and behavioral stress responses. The expression of GR peptide in the brain did not vary with body condition. We also found that while females responded to capture stress by significantly increasing plasma corticosterone, the response did not vary with body condition. Combined with results from prior studies, these data suggest that body condition does not consistently influence stress reactivity or negative feedback regulation of the HPA axis. Female reproductive behavior following capture stress also did not vary with body condition, but did vary with reproductive condition. Parturient females mated more quickly than post-parturient females following capture stress treatment, suggesting that proceptivity during the spring is linked to reproductive history, regardless of body condition. Lastly, females that were later unreceptive to mating increased corticosterone in response to capture stress, but receptive females did not, supporting the idea that receptivity is influenced by reactivity of the HPA axis. These results suggest that HPA axis activity influences reproductive 
"decisions" by modulating receptivity, while proceptivity is related primarily to recent reproductive history.

Circulating glucocorticoids can inhibit their own release from the HPA axis via negative feedback regulation. Thus, the magnitude of the glucocorticoid stress response is partly determined by the strength of negative feedback that glucocorticoids exert on the hypothalamus and hippocampus. In general, higher GR expression in the brain yields stronger negative feedback, which manifests as a smaller magnitude and shorter duration of glucocorticoid stress responses (Krause et al., 2015; Mizoguchi et al., 2003; Ronchi et al., 1998). Beyond the role of genomic low-affinity GR in regulating negative feedback effects, the expression of GR in other brain regions mediates the effects of glucocorticoids on physiology and behavior (Boyle et al., 2005; Chiba et al., 2012; Korte et al., 1995; Tronche et al., 1999; Welberg et al., 2001). For example, chronic restraint stress was associated with decreased GR mRNA expression in the pre-frontal cortex and increased anxiety-like and depression-like behaviors in male rats (Chiba et al., 2012). GR-mediated effects on behavior have also been described in fish, amphibians and birds (Crespi and Denver, 2004; DiBattista et al., 2006, 2005; Dunlap et al., 2011; Horton and Holberton, 2009; Koch et al., 2002; Landys et al., 2004a, 2004b; Soares et al., 2014). We previously showed that mating behavior of red-sided garter snakes is inhibited with lower doses of exogenous corticosterone in females with a negative body condition index compared to those with a positive body condition index (Dayger et al., 2013). Based on these previous results, we hypothesized that females in negative body condition would have more GR peptide in the brain. Our results did not support this hypothesis: we 
found no significant difference in the expression of GR peptide in the brains of females in positive body condition compared to those in negative body condition. However, a potential limitation of this study is that we measured whole brain GR, which cannot account for region-specific variation in GR. Future studies using microdissection of brain regions or immunohistochemistry, in combination with larger sample sizes, are needed to determine if the expression of GR varies with body condition within specific brain regions (e.g., hypothalamus and hippocampus versus the amygdala or other brain regions that mediate the effects of glucocorticoids on behavior).

Dayger et al. (2013) reported that females in negative body condition exhibit a larger stress-induced glucocorticoid increase than females in positive body condition. However, in the current study, female glucocorticoid stress responses did not vary significantly with body condition. Previous studies in reptiles and birds indicate that glucocorticoid stress responses can vary with body condition (Busch and Hayward, 2009; Dayger et al., 2013; Dunlap and Wingfield, 1995; Jessop et al., 2002; Kitaysky et al., 1999; Moore and Jessop, 2003; Müller et al., 2011; Smith et al., 1994; Vitousek et al., 2010), but other studies, including this one, report no significant relationship between body condition and stress-induced glucocorticoid concentrations (Cease et al., 2007; Klukowski, 2011; Moore et al., 2000a; Schmidt et al., 2012; Williams et al., 2008). Significant annual variation in baseline and stress-induced glucocorticoids exists in male red-sided garter snakes (Lutterschmidt and Mason, 2005), but few studies have described stress responses in female red-sided garter snakes. Ouyang et al. (2011) demonstrated that two species of birds have repeatable baseline glucocorticoid concentrations between 
years, but when the authors probed further, baseline glucocorticoid measures were only repeatable during the breeding season, and not between years more generally. It is possible that female red-sided garter snakes also have significant variation in glucocorticoid concentrations year-to-year. Annual variation could explain why stressinduced glucocorticoids do not always vary with body condition in females, but further research is needed to understand how and when body condition is mechanistically linked to the HPA axis. Taken together, these data suggest that the role of body condition in determining physiological and behavioral responses to capture stress is subtle and likely context-dependent.

We found that unreceptive females significantly increased glucocorticoids in response to capture stress, but receptive females did not. Elevated glucocorticoids can suppress mating behavior in many taxa (Landys et al., 2006), including female red-sided garter snakes (Dayger et al., 2013). Thus, elevated stress-induced glucocorticoid concentrations may have rendered some females unreceptive to mating. Alternatively, receptive females may be hormonally insensitive to capture stress. Our data point to the latter possibility because females that did not initially mate in the post-stress behavior trials were given additional opportunities to mate on successive days, yet most remained unreceptive. Stress-induced glucocorticoid concentrations of male red-sided garter snakes return to baseline within 24 hours of capture stress treatment (Moore et al., 2001), suggesting that female plasma glucocorticoids probably also declined to baseline by the next time they were offered an opportunity to mate. Similar to what we observed, unreceptive female Galapagos marine iguanas (Amblyrhynchus cristatus) also have 
higher maximum stress-induced glucocorticoid concentrations than receptive females (Vitousek et al., 2010). Future research is needed to understand why some females are unreceptive despite being apparently able to reproduce.

We previously reported that the latency to copulate varies with body condition in female red-sided garter snakes (Dayger et al., 2013), but we did not observe a significant relationship between female body condition and either receptivity or proceptivity in this study. Note that the current study assessed latency to copulate only in females that mated during the behavior trial, while Dayger et al. (2013) included both receptive and unreceptive females by using a rank-based analysis. This different methodology could explain why the relationship between female reproductive behavior and body condition did not reach significance in this study. We posited that capture stress would cause a female with low body condition to suppress reproductive behavior, via either a large and/or sustained glucocorticoid stress response or by some other mechanism (Romero and Wikelski, 2001). We did not find such a pattern, because maximum stress-induced glucocorticoids and latency to copulate did not differ between females in positive and negative body condition. Interestingly, 13 of the 30 female snakes that mated during the behavior trials in this study were in negative body condition, and 4 of those gave birth to young. Indeed, body condition index did not differ significantly between parturient and post-parturient snakes, primarily due to the large amount of variation observed in body condition among females. Thus, female body condition during the spring mating season does not guarantee a particular reproductive outcome during the summer.

If body condition during the spring is not predictive of a female's later 
reproductive outcome, can female mating behavior predict reproductive condition? We found that parturient females were more proceptive following capture stress treatment than post-parturient females. All the females in our study were courted avidly by males in the behavior trials, and hence the differences in latency to copulate that we observed among females cannot be attributed to differences in female attractivity. Our data therefore indicate that female mating behavior at spring emergence may be linked to recent reproductive history. The physiological mechanisms behind such a relationship are unknown. In marmosets, female proceptivity and receptivity are initially low just after parturition but increase with time, suggesting that recent reproductive history and reproductive behavior may be related across vertebrates (Dixson and Lunn, 1987). It is possible that a change in maternal HPA axis sensitivity could suppress proceptivity in females that have recently given birth until the female has regained sufficient energy stores for the next bout of reproduction. Although female red-sided garter snakes are capital breeders that produce young every other year (Bonnet et al., 1998; Gregory, 2006), body condition at spring emergence was not significantly related to female reproductive condition, receptivity, or proceptivity. Gregory (2006) reported that how much a female feeds while gravid affects its postpartum mass, which could have important implications for a female's reproductive efforts in successive years. It is thus possible that income feeding while gravid will allow some females to reproduce in consecutive years (Gregory, 2006; Whittier and Crews, 1990).

In summary, although capture stress elicited a significant increase in glucocorticoid levels, neither stress-induced glucocorticoids nor post-stress reproductive 
behavior varied with body condition. These results contrast with previous studies, indicating that significant variation in the relationship between body condition and stress responses exists. Unreceptive females had a larger glucocorticoid stress response than receptive females, suggesting that variation in the sensitivity of the HPA axis may contribute to reproductive "decision making." Within receptive females, we found that parturient and post-parturient snakes did not differ significantly in body condition or glucocorticoid stress responses, but parturient females mated more quickly, indicating that female proceptivity may be associated with recent reproductive history. This study sheds light on the role of the HPA axis in modulating reproductive decisions. A better understanding of the physiological mechanisms that females use to make the high-stakes tradeoffs between investing in reproduction versus self-maintenance will be essential for directing well-informed conservation efforts.

\section{ACKNOWLEDGEMENTS}

We thank the Manitoba Department of Conservation and Dave Roberts for logistical support in Manitoba, Canada and Ashley R. Lucas for assistance in the field. We also thank William V. Joersz for his assistance in the lab and especially Bradley A. Buckley and Jason E. Podrabsky for sharing their advice and equipment while we developed the Western blot assay for glucocorticoid receptor. This work was supported in part by a Grant-in-Aid of Research from the Society for Integrative and Comparative Biology and a Forbes-Lea Award from Portland State University to C.A.D. and NSF grant IOS - 1355203 to D.I.L. 


\section{Chapter 6 - Discussion and Conclusions}

The hypothalamus-pituitary-adrenal (HPA) axis is crucial in helping animals cope with environmental challenges. Specifically, animals respond to stressors in their environment by secreting glucocorticoid hormones from the adrenal glands. Stressinduced glucocorticoid hormones coordinate a suite of physiological and behavioral changes to promote survival, including suppression of non-essential processes like reproduction and digestion and modulation of metabolism and the immune system. However, these changes vary in magnitude with sex, season, reproductive state, and energy balance, often via poorly understood mechanisms (Astheimer et al., 1995; Heath and Dufty, 1998; Kitaysky et al., 1999; Schoech et al., 1997; Zerani et al., 1991). Baseline glucocorticoids are also well-known to vary seasonally across taxa (Romero, 2002). Such widespread variation in baseline and stress-induced glucocorticoid concentrations points to corresponding variation in HPA axis activity, but why does HPA axis activity vary, and what role might this variation play?

Because glucocorticoids mobilize energy stores, one possibility is that baseline glucocorticoid concentrations are elevated to fuel periods of high energetic demand (Cornelius et al., 2013; Landys et al., 2006). Baseline glucocorticoids are higher during migration in a number of bird and fish species (Cornelius et al., 2013; Landys et al., 2004c; Landys-Ciannelli et al., 2002; Piersma et al., 2000) as well as the breeding season in birds, reptiles, amphibians and some mammals (Iwata, 1995; Landys et al., 2006; Romero, 2002). For example, baseline glucocorticoids are higher in several species of 
calling male frogs compared to non-calling males (Leary, 2009; Leary et al., 2004; Leary and Harris, 2012). Leary et al. (2004) suggested that elevated glucocorticoids in calling male Great Plains toads (Bufo cognatus) and Woodhouse's toads (Bufo woodhousii) contributed to depletion of energy reserves during vocalization, leading to males switching to the less energetically-expensive satellite tactic of securing mating opportunities. This example illustrates that elevated glucocorticoids are associated with increased energy expenditure and the mediation of a trade-off between behavioral tactics. Could variation in HPA axis activity be fueling the changing energetic demands animals experience between seasons?

\section{Is HPA axis activity related to internal energy stores?}

Because glucocorticoids mobilize energy stores, HPA axis activity could be associated with the amount of internal energy stores an animal possesses. Researchers often describe energy balance as the relationship between an animal's total body mass or the size of a specific fat deposit and its body size, generally referred to as body condition. In snakes and some other ectotherms, body condition is calculated from the relationship between body length and body mass. If an animal is heavier than expected for its length, it is said to be in high body condition with higher-than-average internal energy stores. Previous studies in reptiles and birds indicate that glucocorticoid stress responses vary with body condition (Busch and Hayward, 2009; Dunlap and Wingfield, 1995; Jessop et al., 2002; Kitaysky et al., 1999; Moore and Jessop, 2003; Müller et al., 2011; Smith et al., 1994; Vitousek et al., 2010). For example, body condition and baseline glucocorticoids 
are negatively correlated during the mating season in red-spotted garter snakes, (Thamnophis sirtalis concinnus) (Moore et al., 2000b). In black-legged kittiwakes (Rissa tridactyla) that breed near the Arctic Circle, body condition is inversely related to both baseline and stress-induced glucocorticoid levels (Kitaysky et al., 1999). However, other studies report no significant relationship between body condition and stress-induced glucocorticoid concentrations (Cease et al., 2007; Klukowski, 2011; Moore et al., 2000a; Schmidt et al., 2012; Williams et al., 2008).

In red-sided garter snakes, some of my studies indicate that variation in HPA axis activity is related to body condition. In Chapter 4 (body condition experiment), I found that during two different field seasons, hormonal and behavioral responses to capture stress and behavioral responses to exogenous glucocorticoids varied with body condition in females (Dayger et al., 2013). Although baseline glucocorticoids did not differ between females in negative or positive body condition, my data showed that female snakes in negative body condition were more sensitive to the effects of capture stress and exogenous glucocorticoids. For example, the observed capture stress-induced increase in glucocorticoids was driven primarily by females in negative body condition. In addition, body condition, but not capture stress treatment, influenced latency to copulate, suggesting that females were resistant to the behavioral effects of capture stress to some degree. Furthermore, only females in negative body condition increased latency to copulate in response to the low dose of exogenous glucocorticoids, while all females responded to the high dose of glucocorticoids (Dayger et al., 2013).

In males, baseline glucocorticoid levels were negatively correlated with body 
condition (Moore et al., 2001). Thus, there is some evidence in red-sided garter snakes to support a relationship between body condition and HPA axis activity. However, other studies of red-sided garter snakes, including the female data presented in Chapter 2 (ACTH challenge experiment) and Chapter 5 (reproductive history experiment), indicated that body condition was not significantly related to HPA axis activity. In female red-sided garter snakes, the glucocorticoid response to adrenocorticotropic hormone (ACTH) did not vary explicitly with body condition category (Dayger and Lutterschmidt, 2016). In Chapter 5 (reproductive history experiment), the expression of GR peptide in the brain did not vary with body condition, and while females responded to capture stress by significantly increasing plasma corticosterone, the response did not vary with body condition (Dayger et al., in review). Taken together, these data suggest that the relationship between body condition and HPA axis activity is likely subtle and contextdependent.

One explanation for the context-dependent relationship between body condition and HPA axis activity is that the calculation of a body condition index may not accurately reflect energy stores (Jakob et al., 1996; Peig and Green, 2009). For example, in male red-sided garter snakes, the majority of the variation in body mass was attributable to musculo-skeletal mass rather than the size of fat bodies, which were relatively small during the spring (Shine and Mason, 2005). Indeed, plasma leptin, a hormone that is positively correlated with internal fat stores in mammals (Houseknecht et al., 1998), is not related to body condition index in either male or female red-sided garter snakes (Wilson and Lutterschmidt, 2017), suggesting that our measure of body condition may 
not be closely related to the amount of fat stores a snake carries. Another possibility is that the trend in body condition over time could be a more informative signal to the animal than body condition at a certain moment. For example, the rate of weight gain postpartum in beef cows affected calf weight more than primiparous body condition index (Spitzer et al., 1995). There are other examples of endocrine-mediated changes in physiology and behavior that depend more on the rate of change than the absolute magnitude at any particular time point. For example, it is the increase in day length over time that serves as the critical signal to induce seasonal reproduction in Syrian hamsters (Reiter, 1993; Skene et al., 1987). Thus, it may be the rate of increasing or decreasing body condition index over time that is the signal most closely related to HPA axis activity, a possibility that could be easily tested and should be investigated further.

\section{Does HPA axis activity fuel migration?}

Periods of high energetic demand, such as migration, are often associated with high body condition, suggesting that elevated baseline glucocorticoids actually mobilize the energy stores animals use to meet high energetic demands (Cornelius et al., 2013; Landys et al., 2006). For example, baseline glucocorticoids are higher during migratory flight in a number of bird species (Landys et al., 2004c; Landys-Ciannelli et al., 2002;

Piersma et al., 2000). In red-sided garter snakes, the relationship between high baseline glucocorticoids and migration may vary seasonally. During the spring, male red-sided garter snakes have lower baseline and similar stress-induced glucocorticoid levels when they have begun to migrate away from the breeding grounds during the spring (Cease et 
al., 2007; Maine et al., 2014). Therefore, during the spring mating season, HPA axis activity shifts such that baseline glucocorticoid levels decline. During the initial stages of migration, females had similar baseline and stress-induced glucocorticoid concentrations compared to pre-migratory females during the spring, although the timing of when the peak stress-induced concentration was reached occurred at a later sampling time in migrating females (Dayger and Lutterschmidt, 2017). However, one preliminary report found that migrating females had lower baseline glucocorticoids than pre-migratory females, similar to what has been reported in males (Maine et al., 2014). What is consistent between the two reports is that HPA axis activity is changing as females begin to migrate.

During fall migration, females had elevated baseline glucocorticoids compared to females during the spring, and experienced no significant stress-induced increase in glucocorticoids (Dayger and Lutterschmidt, 2017). The migration modulation hypothesis, first proposed in birds, describes a similar migration-specific pattern of glucocorticoid release characterized by elevated baseline corticosterone and the lack of a significant stress-induced increase in corticosterone (Holberton et al., 1996). My findings in female snakes during fall migration, but not spring migration, support the migration modulation hypothesis. Similar differences in the stress responses of yellow-rumped warblers (Dendroica coronata) during spring and fall migration were reported by Holberton (1999). Holberton (1999) suggested that the variation resulted from seasonal differences in energetic condition because birds were leanest during spring migration and fattest during fall migration. As in yellow-rumped warblers, red-sided garter snakes also have a 
lower body condition index during the spring compared to fall (e.g., Moore et al., 2001), and therefore seasonal differences in stress responses during migration could be related to seasonal differences in energetic condition.

\section{Does HPA axis activity influence the onset of migration?}

Males and females generally spend different amounts of time at the den during the spring mating season. Males emerge earlier in the season and stay at the den for up to 2 weeks (Shine et al., 2001). Females emerge later in the season and remain at the den for approximately 24 hours (Gregory, 1974; Shine et al., 2001). This sex difference in life history results in a difference in the duration of the mating season for males and females. One possible mechanism underlying this sex difference is a change in HPA axis activity mediating a shift in behavior from mating to migration (Lutterschmidt and Maine, 2014).

With data from Chapters 2 and 3, I had the opportunity to uncover potential sex differences. Following a capture stress methodology identical to the one employed in my studies, pre-migratory males in the spring did not respond to capture stress with a significant increase in glucocorticoids over baseline levels (Cease et al., 2007; Moore et al., 2001). However, studies using different sampling regimes yielded different results (Lutterschmidt and Mason, 2010, 2005; Moore et al., 2000a), and further examination is needed to confirm the sex difference implicated by my new results: that pre-migratory females remain sensitive to capture stress during the spring mating season, while males have reduced stress sensitivity. In support of this hypothesis, males are significantly less responsive to ACTH during the spring than females (Dayger and Lutterschmidt, 2016). 
Considering that sex differences in the timing of migration are fairly common (Francis and Cooke, 1986), it is possible that differences in HPA axis activity underlie sex differences in the onset of migration (and therefore the duration of mating activity) in other taxa with a similar life-history pattern.

In many animals, HPA axis activity can change over the course of the mating season, suggesting that changing HPA axis activity could actually mediate the transition between reproduction and other life-history stages. For example, corticosterone stress responses differed between Lapland longspurs (Calcarius lapponicus) that had just completed migration to the breeding grounds and those that had been at the breeding grounds for several weeks, supporting migration-related modulation of the HPA axis (Walker et al., 2015). In male red-sided garter snakes, Cease et al. (2007) reported that baseline glucocorticoids decline over the course of the mating season and are significantly lower in snakes beginning to migrate away from the den site; therefore stress-induced changes in glucocorticoid concentrations were significant only in migrating snakes. As mentioned previously, plasma glucocorticoids in pre-migratory females responded to capture stress and returned to baseline more quickly compared to migratory females. These results suggest that seasonal changes in HPA axis regulation are initiated as soon as males and females begin to migrate from the spring breeding grounds. To demonstrate that changing HPA axis activity actually mediates this lifehistory transition during the spring, manipulation of glucocorticoids is required.

Experimentally maintaining elevated glucocorticoids may extend the duration of the mating season, which would support the hypothesis that declining glucocorticoid 
concentrations mediate the transition to migratory behavior. Because of the natural history of re-sided garter snakes, it is currently not feasible to track individual snakes as they migrate away from the den site, and thus it is not possible to determine if individual HPA axis activity is correlated with migratory behavior. Advances in the miniaturization of GPS trackers in recent years now allows individual tracking of smaller-bodied animals more readily than ever before (Jønsson et al., 2016). In the future, it may therefore be possible to track and re-capture individual snakes through life-history transitions, which would permit a more in-depth longitudinal study of the role of glucocorticoids in mediating life-history transitions.

It is possible that strong negative feedback on the HPA axis dampened the corticosterone stress response in pre-migratory females during the spring. Circulating glucocorticoids feed back on the HPA axis to suppress their own release (Whitnall, 1993). Lattin et al. (2012) reported that negative feedback was stronger in house sparrows (Passer domesticus) during the breeding season compared to the post-breeding molting season. Similar to the pattern observed in house sparrows, the strength of negative feedback on the HPA axis may be stronger when female red-sided garter snakes are at the breeding grounds during the spring and then weaken as females transition to migratory behavior and disperse to the summer feeding grounds. Significantly, strong negative feedback would allow animals with limited breeding opportunities to reproduce despite the presence of environmental perturbations and elevated glucocorticoids. One way that the strength of negative feedback could be adjusted is by varying the quantity of glucocorticoid receptors (GR) in the brain. A higher quantity of receptors for 
glucocorticoids would render the brain exquisitely sensitive to even the smallest change in glucocorticoid concentrations. In house sparrows (Passer domesticus), GR mRNA expression changes seasonally (Breuner and Orchinik, 2001) and is associated with the strength of negative feedback (Liebl et al., 2013). The Western blot for GR that I developed in Chapter 5 (reproductive history experiment) makes quantifying the amount of GR protein in pre-migratory and migratory red-sided garter snakes achievable and would allow future research to determine if the strength of negative feedback changes as snakes transition to migratory behavior.

\section{Does changing HPA axis activity modulate reproductive decisions?}

Elevated glucocorticoids are important for the expression of both male and female reproductive behavior in red-sided garter snakes. As mentioned previously, variation in HPA axis activity may be related to how long a snake engages in mating behavior during the spring mating season. Put another way, variation in HPA axis activity can impact "decisions" about if, when, and how long to engage in reproductive behavior, with consequent impacts on reproductive fitness.

Baseline glucocorticoids are elevated during the spring mating season in male red-sided garter snakes, while sex-steroid hormones are generally low, suggesting a larger role for glucocorticoids than sex-steroids in supporting reproductive behavior during the spring in males (Krohmer and Lutterschmidt, 2011; Lutterschmidt, 2012). Further support for elevated glucocorticoids supporting mating behavior in males comes from several reports of baseline glucocorticoids declining over the course of the breeding 
season before males finally migrate away from the den site to seek feeding opportunities (Cease et al., 2007; Krohmer et al., 1987; Lutterschmidt and Maine, 2014). Declining circulating glucocorticoids occurs concurrently with declining intensity of male courtship behavior (Lutterschmidt and Maine, 2014). Lutterschmidt and Maine (2014) also showed that experimentally decreasing glucocorticoids stimulates males to prematurely transition to feeding behavior, a hallmark of the end of the mating season. These data further support the idea that elevated baseline glucocorticoids are required for the maintenance of courtship behavior in male red-sided garter snakes. A study where elevated glucocorticoids are maintained experimentally is needed to determine if the duration of the mating season for males could be extended.

If glucocorticoid concentrations rise above a threshold, such as when exogenous glucocorticoids are administered, mating behavior can be suppressed in males and females of many taxa (e.g., Dayger et al., 2013; Lutterschmidt et al., 2004; Moore and Miller, 1984; Moore and Mason, 2001; Salvante and Williams, 2003; Wingfield and Sapolsky, 2003). For example, female zebra finches (Taeniopygia guttata) are significantly less likely to initiate egg laying when treated with implants that release glucocorticoids (Salvante and Williams, 2003). In another example, injection of exogenous glucocorticoids decreased sexual behavior in male rough-skinned newts in a dose-dependent manner (Moore and Miller, 1984). Taken together, these data suggest that there are times when the survival-promoting benefits of glucocorticoids outweigh the fitness-promoting benefits of suppressing responses to a stressor (i.e., elevated glucocorticoids). These data also suggest that there is range of glucocorticoids that 
supports reproductive behavior, and deviations from that range result in suppression of behavior (Lutterschmidt, 2012). Consequently, plasticity in HPA axis activity may arise to protect activities such as mating behavior when baseline glucocorticoids are already high (Wingfield and Sapolsky, 2003).

Glucocorticoids may be important modulators of female reproductive behavior as they are in males. In Chapter 5 (reproductive history experiment), I report that only unreceptive females showed a significant stress-induced increase in glucocorticoids, indicating that HPA axis activity is associated with receptivity (Dayger et al., in review). Elevated glucocorticoids can suppress mating behavior in many taxa (Landys et al., 2006), including female red-sided garter snakes (Dayger et al., 2013). Thus, elevated stress-induced glucocorticoid concentrations may have rendered some females unreceptive to mating (Dayger et al., 2013). Alternatively, receptive females may be hormonally insensitive to capture stress. Our data suggest the latter possibility because females that did not initially mate in the post-stress behavior trials were given additional opportunities to mate on successive days, yet most remained unreceptive. Stress-induced glucocorticoid concentrations of male red-sided garter snakes return to baseline within 24 hours of capture stress treatment (Moore et al., 2001), and thus female plasma glucocorticoids probably also declined to baseline by the next time they were offered an opportunity to mate. Similar to what I observed, unreceptive female Galapagos marine iguanas (Amblyrhynchus cristatus) also have higher maximum stress-induced glucocorticoid concentrations than receptive females (Vitousek et al., 2010). These data suggest that HPA axis activity modulates receptivity. Future research is needed to 
understand why some females are unreceptive despite being apparently able to reproduce.

In Chapter 5 (reproductive history experiment), I also found that proceptivity, but not receptivity, was related to reproductive history. Females that gave birth during the summer, and thus likely did not reproduce the previous summer, mated more quickly than females that did not give birth (Dayger et al., in review). These data imply that proceptivity could be modulated prior to the mating season, perhaps in the previous fall pre-dormancy period or during winter dormancy. Such trans-seasonal carry-over effects on reproduction have been observed in birds. For example, in American redstarts (Setophaga ruticilla), poor quality overwintering habitat was associated with higher baseline glucocorticoids, a later migration departure date and lower reproductive success in the subsequent summer (Marra et al., 1998). Preliminary data in red-sided garter snakes suggest that physiological changes during winter dormancy could impact reproductive behavior in the spring. For example, gonadotropin releasing hormone $(\mathrm{GnRH})$ is an important regulator of reproductive physiology and behavior (Gore, 2002). In male, but not female red-sided garter snakes, GnRH cell size and cell number increase over the course of winter dormancy, and these changes may induce male courtship behavior upon spring emergence (Lutterschmidt, 2015). Further research is needed to understand if and how changes in HPA axis activity and glucocorticoid concentrations during winter dormancy impact reproductive behavior during the spring.

\section{Conclusions}

I have shown that HPA axis activity is highly context-dependent, varying with 
body condition in some cases as well as with sex, season, and reproductive history. Each of these contexts is related to the seasonal life-history transition between reproduction, migration, and the subsequent activation of feeding behavior. There is growing support for a role for glucocorticoids in coordinating transitions between life-history stages (Ketterson et al., 2015; Lattin et al., 2016; Ramenofsky and Wingfield, 2017; Wada, 2008). My dissertation research has increased what is known about the mechanisms underlying life-history transitions, especially in females, using the well-studied red-sided garter snake. This new information is critical to illuminating potential sex differences, which can highlight differing selection pressures on males and females. However, when sex differences do not exist, knowing males and females are similar points to more broadly applicable physiological mechanisms. Just as comparative studies can uncover common mechanisms across taxa, comparing males and females can uncover evolutionarily conserved mechanisms among sexually reproducing species. Glucocorticoids from the HPA axis are commonly viewed as primarily stress hormones because of their important role in stress responses. In other instances glucocorticoids are viewed as primarily metabolic hormones due to their ability to mobilize energy stores to meet energetic demands. In many ways, glucocorticoids are also reproductive hormones important for supporting mating behavior and reproductive decisions. The common thread is that glucocorticoids mediate trade-offs: between physiological states and between behaviors. In each case, glucocorticoids assist the animal in navigating the balancing act between the benefits of engaging in a certain activity compared to the cost of not engaging in a different activity. When resources are limited, which they are for all 
animals at least some of the time, animals, including humans, must make choices about what activities they devote energy toward. Glucocorticoids are an essential tool that allow animals to align their physiology and behavior to a particular activity, theoretically based on what activity will provide the greatest overall fitness benefit. A better understanding of the role of the HPA axis in mediating trade-offs allows a clearer view of how animals make decisions under changing conditions. These studies investigating the role of the HPA axis in the seasonal biology of red-sided garter snakes shed light on how and why all animals do what they do, when they do it. Populations are increasingly threatened by changing climate, habitat loss, invasive species, and pollutants, perturbations that are all caused or exacerbated by human activities. A solid understanding of the mechanisms that animals use to integrate information about their environment, synchronize their physiology and behavior appropriately, and transition between life-history stages will be important for protecting vulnerable populations. This information will be critical if humans are to make informed management and conservation decisions to ensure persistence of the ecological systems we threaten. 


\section{Chapter 1 References}

Astheimer, L.B., Buttemer, W.A., Wingfield, J.C., 1995. Seasonal and acute changes in adrenocortical responsiveness in an arctic-breeding bird. Horm. Behav. 29, 442457. doi:10.1006/hbeh.1995.1276

Belda, X., Daviu, N., Nadal, R., Armario, A., 2012. Acute stress-induced sensitization of the pituitary-adrenal response to heterotypic stressors: Independence of glucocorticoid release and activation of CRH1 receptors. Horm. Behav. 62, 515524. doi:10.1016/j.yhbeh.2012.08.013

Birkhead, T.R., Møller, A.P., 1998. Sperm competition and sexual selection. Academic Press.

Bonnet, X., Bradshaw, D., Shine, R., 1998. Capital versus Income Breeding: An Ectothermic Perspective. Oikos 83, 333-342. doi:10.2307/3546846

Breuner, C.W., Delehanty, B., Boonstra, R., 2013. Evaluating stress in natural populations of vertebrates: total CORT is not good enough. Funct. Ecol. 27, 2436. doi:10.1111/1365-2435.12016

Breuner, C.W., Orchinik, M., 2002. Plasma binding proteins as mediators of corticosteroid action in vertebrates. J. Endocrinol. 175, 99-112.

Breuner, C.W., Orchinik, M., 2001. Seasonal regulation of membrane and intracellular corticosteroid receptors in the house sparrow brain. J. Neuroendocrinol. 13, 412420.

Cartledge, V.A., Jones, S.M., 2007. Does adrenal responsiveness vary with sex and reproductive status in Egernia whitii, a viviparous skink? Gen. Comp. Endocrinol. 150, 132-139. doi:10.1016/j.ygcen.2006.07.021

Cease, A.J., Lutterschmidt, D.I., Mason, R.T., 2007. Corticosterone and the transition from courtship behavior to dispersal in male red-sided garter snakes (Thamnophis sirtalis parietalis). Gen. Comp. Endocrinol. 150, 124-131.

Crews, D., 1984. Gamete production, sex hormone secretion, and mating behavior uncoupled. Horm. Behav. 18, 22-28.

Crews, D., Grassman, M., Garstka, W.R., Halpert, A., Camazine, B., 1987. Sex and seasonal differences in metabolism in the red-sided garter snake, Thamnophis sirtalis parietalis. Can. J. Zool. 65, 2362-2368. doi:10.1139/z87-357

Dayger, C.A., Cease, A.J., Lutterschmidt, D.I., 2013. Responses to capture stress and exogenous corticosterone vary with body condition in female red-sided garter snakes (Thamnophis sirtalis parietalis). Horm. Behav. 64, 748-754. doi: 10.1016/j.yhbeh.2013.09.003

Dickens, M., Romero, L.M., Cyr, N.E., Dunn, I.C., Meddle, S.L., 2009. Chronic stress alters glucocorticoid receptor and mineralocorticoid receptor mRNA expression in the European starling (Sturnus vulgaris) brain. J. Neuroendocrinol. 21, 832-840. doi:10.1111/j.1365-2826.2009.01908.x

Drent, R.H., Daan, S., 1980. The prudent parent: energetic adjustments in avian breeding. Ardea 68, 225-252.

Dunlap, K.D., Wingfield, J.C., 1995. External and internal influences on indices of physiological stress. I. Seasonal and population variation in adrenocortical 
secretion of free-living lizards, Sceloporus occidentalis . J. Exp. Zool. 271, 36-46. doi:10.1002/jez.1402710105

Garstka, W.R., Camazine, B., Crews, D., 1982. Interactions of behavior and physiology during the annual reproductive cycle of the red-sided garter snake (Thamnophis sirtalis parietalis). Herpetologica 104-123.

Ghisleni, G., Capiotti, K.M., Da Silva, R.S., Oses, J.P., Piato, Â.L., Soares, V., Bogo, M.R., Bonan, C.D., 2012. The role of CRH in behavioral responses to acute restraint stress in zebrafish. Prog. Neuropsychopharmacol. Biol. Psychiatry 36, 176-182. doi:10.1016/j.pnpbp.2011.08.016

Girard, C., Brodeur, J.C., Hontela, A., 1998. Responsiveness of the interrenal tissue of yellow perch (Perca flavescens) from contaminated sites to an ACTH challenge test in vivo. Can. J. Fish. Aquat. Sci. 55, 438-450. doi:10.1139/197-224

Gregory, P.T., 1977. Life-history parameters of the red-sided garter snake (Thamnophis sirtalis parietalis) in an extreme environment, the Interlake Region of Manitoba. National Museums of Canada, Ottawa.

Gregory, P.T., 1974. Patterns of spring emergence of the red-sided garter snake (Thamnophis sirtalis parietalis) in the Interlake region of Manitoba. Can. J. Zool. 52, 1063-1069. doi:10.1139/z74-141

Gregory, P.T., Stewart, K.W., 1975. Long-distance dispersal and feeding strategy of the red-sided garter snake (Thamnophis sirtalis parietalis) in the Interlake of Manitoba. Can. J. Zool. 53, 238-245. doi:10.1139/z75-030

Heath, J.A., Dufty, J., 1998. Body condition and the adrenal stress response in captive American kestrel juveniles. Physiol. Biochem. Zool. 71, 67-73.

Iwata, M., 1995. Downstream migratory behavior of salmonids and its relationship with cortisol and thyroid hormones: A review. Aquaculture, Application of Endocrinology to Pacific Rim Aquaculture 135, 131-139. doi:10.1016/00448486(95)01000-9

Jacobs, J.D., 1996. Regulation of life history strategies with individuals in predictable and unpredictable environments (Thesis).

Katz, A., Mirzatoni, A., Zhen, Y., Schlinger, B.A., 2008. Sex differences in cell proliferation and glucocorticoid responsiveness in the zebra finch brain. Eur. J. Neurosci. 28, 99-106. doi:10.1111/j.1460-9568.2008.06303.x

Kitaysky, A.S., Wingfield, J.C., Piatt, J.F., 1999. Dynamics of food availability, body condition and physiological stress response in breeding Black-legged Kittiwakes. Funct. Ecol. 13, 577-584. doi:10.1046/j.1365-2435.1999.00352.x

Krohmer, R.W., Grassman, M., Crews, D., 1987. Annual reproductive cycle in the male red-sided garter snake, Thamnophis sirtalis parietalis: field and laboratory studies. Gen. Comp. Endocrinol. 68, 64-75.

Krohmer, R.W., Lutterschmidt, D.I., 2011. Environmental and neuroendocrine control of reproduction in snakes, in: Aldridge, R.D., Seaver, D.M. (Eds.), Reproductive Biology and Phylogeny of Snakes. pp. 289-346.

Ladd, C.O., Huot, R.L., Thrivikraman, K.V., Nemeroff, C.B., Plotsky, P.M., 2004. Longterm adaptations in glucocorticoid receptor and mineralocorticoid receptor mrna and negative feedback on the hypothalamo-pituitary-adrenal axis following 
neonatal maternal separation. Biol. Psychiatry 55, 367-375.

doi:10.1016/j.biopsych.2003.10.007

Landys, M.M., Ramenofsky, M., Wingfield, J.C., 2006. Actions of glucocorticoids at a seasonal baseline as compared to stress-related levels in the regulation of periodic life processes. Gen. Comp. Endocrinol. 148, 132-149.

doi:10.1016/j.ygcen.2006.02.013

Liu, D., Diorio, J., Tannenbaum, B., Caldji, C., Francis, D., Freedman, A., Sharma, S., Pearson, D., Plotsky, P.M., Meaney, M.J., 1997. Maternal Care, Hippocampal Glucocorticoid Receptors, and Hypothalamic-Pituitary-Adrenal Responses to Stress. Science 277, 1659-1662. doi:10.1126/science.277.5332.1659

Lutterschmidt, D., 2015. Sex or candy? Neuroendocrine regulation of seasonal lifehistory transitions. Presented at the North American Society for Comparative Endocrinology, Ottawa, Ontario, Canada.

Lutterschmidt, D.I., 2012. Chronobiology of reproduction in garter snakes: Neuroendocrine mechanisms and geographic variation. Gen. Comp. Endocrinol. 176, 448-455. doi:10.1016/j.ygcen.2011.12.015

Lutterschmidt, D.I., LeMaster, M.P., Mason, R.T., 2004. Effects of melatonin on the behavioral and hormonal responses of red-sided garter snakes (Thamnophis sirtalis parietalis) to exogenous corticosterone. Horm. Behav. 46, 692-702. doi:10.1016/j.yhbeh.2004.06.013

Lutterschmidt, D.I., Maine, A.R., 2014. Sex or candy? Neuroendocrine regulation of the seasonal transition from courtship to feeding behavior in male red-sided garter snakes (Thamnophis sirtalis parietalis). Horm. Behav. 66, 120-134. doi:10.1016/j.yhbeh.2014.01.011

Lutterschmidt, D.I., Mason, R.T., 2010. Temporally distinct effects of stress and corticosterone on diel melatonin rhythms of red-sided garter snakes (Thamnophis sirtalis). Gen. Comp. Endocrinol. 169, 11-17. doi:10.1016/j.ygcen.2010.06.013

Lutterschmidt, D.I., Mason, R.T., 2005. A serotonin receptor antagonist, but not melatonin, modulates hormonal responses to capture stress in two populations of garter snakes (Thamnophis sirtalis parietalis and Thamnophis sirtalis concinnus). Gen. Comp. Endocrinol. 141, 259-270. doi:10.1016/j.ygcen.2005.01.012

Mommsen, T.P., Vijayan, M.M., Moon, T.W., 1999. Cortisol in teleosts: dynamics, mechanisms of action, and metabolic regulation. Rev. Fish Biol. Fish. 9, 211-268. doi:10.1023/A:1008924418720

Moore, F.L., Miller, L.J., 1984. Stress-induced inhibition of sexual behavior: Corticosterone inhibits courtship behaviors of a male amphibian (Taricha granulosa). Horm. Behav. 18, 400-410. doi:10.1016/0018-506X(84)90026-6

Moore, I.T., Greene, M.J., Mason, R.T., 2001. Environmental and seasonal adaptations of the adrenocortical and gonadal responses to capture stress in two populations of the male garter snake, Thamnophis sirtalis. J. Exp. Zool. 289, 99-108.

Moore, I.T., LeMaster, M.P., Mason, R.T., 2000a. Behavioural and hormonal responses to capture stress in the male red-sided garter snake, Thamnophis sirtalis parietalis. Anim. Behav. 59, 529-534. doi:10.1006/anbe.1999.1344

Moore, I.T., Lerner, J.P., Lerner, D.T., Mason, R.T., 2000b. Relationships between 
annual cycles of testosterone, corticosterone, and body condition in male redspotted garter snakes, Thamnophis sirtalis concinnus. Physiol. Biochem. Zool. PBZ 73, 307-312. doi:10.1086/316748

Moore, I.T., Mason, R.T., 2001. Behavioral and hormonal responses to corticosterone in the male red-sided garter snake, Thamnophis sirtalis parietalis. Physiol. Behav. 72, 669-674. doi:10.1016/S0031-9384(01)00413-9

Nichols, T., Nugent, C.A., Tyler, F.H., 1965. Diurnal Variation in Suppression of Adrenal Function by Glucocorticoids. J. Clin. Endocrinol. Metab. 25, 343-349. doi:10.1210/jcem-25-3-343

Paul, M.J., Zucker, I., Schwartz, W.J., 2008. Tracking the seasons: the internal calendars of vertebrates. Philos. Trans. R. Soc. Lond. B Biol. Sci. 363, 341-361. doi:10.1098/rstb.2007.2143

Phillips, J.B., Klukowski, M., 2008. Influence of season and adrenocorticotropic hormone on corticosterone in free-living female Eastern Fence Lizards (Sceloporus undulatus). J. Inf. 2008.

Post, E., Forchhammer, M.C., 2008. Climate change reduces reproductive success of an Arctic herbivore through trophic mismatch. Philos. Trans. R. Soc. Lond. B. Biol. Sci. 363, 2369-2375. doi:10.1098/rstb.2007.2207

Post, E., Forchhammer, M.C., Stenseth, N.C., Callaghan, T.V., 2001. The timing of lifehistory events in a changing climate. Proc. R. Soc. Lond. B Biol. Sci. 268, 15.

Ratka, A., Sutanto, W., Bloemers, M., de Kloet, E.R., 1989. On the Role of Brain Mineralocorticoid (Type I) and Glucocorticoid (Type II) Receptors in Neuroendocrine Regulation. Neuroendocrinology 50, 117-123. doi: $10.1159 / 000125210$

Robertson, B.D., Newman, A.E.M., Macdougall-Shackleton, S.A., 2015. Perils and pitfalls of manipulating glucocorticoids with silicone implants., in: Integrative and Comparative Biology. Oxford Univ Press Inc Journals Dept, 2001 Evans Rd, Cary, NC 27513 USA, pp. E154-E154.

Romero, L.M., 2002. Seasonal changes in plasma glucocorticoid concentrations in freeliving vertebrates. Gen. Comp. Endocrinol. 128, 1-24. doi:10.1016/S00166480(02)00064-3

Romero, L.M., Meister, C.J., Cyr, N.E., Kenagy, G.J., Wingfield, J.C., 2008. Seasonal glucocorticoid responses to capture in wild free-living mammals. Am. J. Physiol. - Regul. Integr. Comp. Physiol. 294, R614-R622. doi:10.1152/ajpregu.00752.2007

Romero, L.M., Soma, K.K., Wingfield, J.C., 1998. Hypothalamic-pituitary-adrenal axis changes allow seasonal modulation of corticosterone in a bird. Am. J. Physiol.Regul. Integr. Comp. Physiol. 274, R1338-R1344.

Romero, L.M., Wikelski, M., 2006. Diurnal and nocturnal differences in hypothalamicpituitary-adrenal axis function in Galápagos marine iguanas. Gen. Comp. Endocrinol. 145, 177-181. doi:10.1016/j.ygcen.2005.09.011

Salvante, K.G., Williams, T.D., 2003. Effects of corticosterone on the proportion of breeding females, reproductive output and yolk precursor levels. Gen. Comp. Endocrinol. 130, 205-214. doi:10.1016/S0016-6480(02)00637-8 
Sapolsky, R.M., 2002. Endocrinology of the stress-response, in: Behavioral Endocrinology. MIT Press, pp. 409-435.

Sapolsky, R.M., 1983. Individual Differences in Cortisol Secretory Patterns in the Wild Baboon: Role of Negative Feedback Sensitivity*. Endocrinology 113, 22632267. doi:10.1210/endo-113-6-2263

Schiml, P.A., Rissman, E.F., 1999. Cortisol facilitates induction of sexual behavior in the female musk shrew (Suncus murinus). Behav. Neurosci. 113, 166-175. doi:10.1037/0735-7044.113.1.166

Schoech, S.J., Mumme, R.L., Wingfield, J.C., 1997. Corticosterone, Reproductive Status, and Body Mass in a Cooperative Breeder, the Florida Scrub-Jay (Aphelocoma coerulescens). Physiol. Zool. 70, 68-73. doi:10.2307/30164285

Schuett, G.W., Grober, M.S., Van Kirk, E.A., Murdoch, W.J., 2004. Long-term sperm storage and plasma steroid profile of pregnancy in a western diamond-backed rattlesnake (Crotalus atrox). Herpetol. Rev. 35, 328-332.

Sever, D.M., Hamlett, W.C., 2001. Female sperm storage in reptiles. J. Exp. Zool. 292, 187-199.

Shine, R., Elphick, M.J., Harlow, P.S., Moore, I.T., LeMaster, M.P., Mason, R.T., 2001. Movements, Mating, and Dispersal of Red-Sided Gartersnakes (Thamnophis sirtalis parietalis) from a Communal Den in Manitoba. Copeia 2001, 82-91.

Shine, R., Mason, R.T., 2005. Do a male garter snake's energy stores limit his reproductive effort? Can. J. Zool. 83, 1265-1270. doi:10.1139/z05-119

Smith, C.F., Schuett, G.W., Hoss, S.K., 2012. Reproduction in female Copperhead snakes (Agkistrodon contortrix): Plasma steroid profiles during gestation and post-birth periods. Zoolog. Sci. 29, 273-279.

Tamarkin, L., Baird, C.J., Almeida, O.F.X., 1985. Melatonin: a coordinating signal for mammalian reproduction. Science 227, 714-721.

Taylor, E.N., DeNardo, D.F., Jennings, D.H., 2004. Seasonal steroid hormone levels and their relation to reproduction in the Western Diamond-backed Rattlesnake, Crotalus atrox (Serpentes: Viperidae). Gen. Comp. Endocrinol. 136, 328-337.

Terzolo, M., Zaggia, B., Allasino, B., De Francia, S., 2014. Practical treatment using mitotane for adrenocortical carcinoma. Curr. Opin. Endocrinol. Diabetes Obes. 21, 159-165. doi:10.1097/MED.0000000000000056

Vitousek, M.N., Mitchell, M.A., Romero, L.M., Awerman, J., Wikelski, M., 2010. To breed or not to breed: physiological correlates of reproductive status in a facultatively biennial iguanid. Horm. Behav. 57, 140-146. doi:10.1016/j.yhbeh.2009.09.020

Walker, B.G., Meddle, S.L., Romero, L.M., Landys, M.M., Reneerkens, J., Wingfield, J.C., 2015. Breeding on the extreme edge: Modulation of the adrenocortical response to acute stress in two High Arctic passerines. J. Exp. Zool. Part Ecol. Genet. Physiol. 323, 266-275. doi:10.1002/jez.1923

Weiss, S.L., Johnston, G., Moore, M.C., 2007. Corticosterone stimulates hatching of lateterm tree lizard embryos. Comp. Biochem. Physiol. A. Mol. Integr. Physiol. 146, 360-365. doi:10.1016/j.cbpa.2006.11.009

Whitnall, M.H., 1993. Regulation of the hypothalamic corticotropin-releasing hormone 
neurosecretory system. Prog. Neurobiol. 40, 573-629. doi:10.1016/03010082(93)90035-Q

Whittier, J.M., Crews, D., 1990. Body Mass and Reproduction in Female Red-Sided Garter Snakes (Thamnophis sirtalis parietalis). Herpetologica 46, 219-226. doi: $10.2307 / 3892907$

Whittier, J.M., Mason, R.T., Crews, D., 1987. Plasma steroid hormone levels of female red-sided garter snakes, Thamnophis sirtalis parietalis: relationship to mating and gestation. Gen. Comp. Endocrinol. 67, 33-43.

Wingfield, J.C., Maney, D.L., Breuner, C.W., Jacobs, J.D., Lynn, S., Ramenofsky, M., Richardson, R.D., 1998. Ecological Bases of Hormone-Behavior Interactions: The "Emergency Life History Stage." Am. Zool. 38, 191-206. doi:10.1093/icb/38.1.191

Wingfield, J.C., Sapolsky, R.M., 2003. Reproduction and Resistance to Stress: When and How. J. Neuroendocrinol. 15, 711-724. doi:10.1046/j.1365-2826.2003.01033.x

Yao, M., Schulkin, J., Denver, R.J., 2008. Evolutionarily Conserved Glucocorticoid Regulation of Corticotropin-Releasing Factor Expression. Endocrinology 149, 2352-2360. doi:10.1210/en.2007-1551

Zerani, M., Amabili, F., Mosconi, G., Gobbetti, A., 1991. Effects of captivity stress on plasma steroid levels in the green frog, Rana esculenta, during the annual reproductive cycle. Comp. Biochem. Physiol. A Physiol. 98, 491-496. doi:10.1016/0300-9629(91)90436-G

\section{Chapter 2 References}

Ashley, N. T., Barboza, P. S., Macbeth, B. J., Janz, D. M., Cattet, M. R. L., Booth, R. K. and Wasser, S. K. (2011). Glucocorticosteroid concentrations in feces and hair of captive caribou and reindeer following adrenocorticotropic hormone challenge. Gen. Comp. Endocrinol. 172, 382-391.

Astheimer, L. B., Buttemer, W. A. and Wingfield, J. C. (1995). Seasonal and acute changes in adrenocortical responsiveness in an arctic-breeding bird. Horm. Behav. 29, 442-457.

Capaldo, A., Gay, F., Valiante, S., Varlese, M. g., Laforgia, V. and Varano, L. (2004). Release of aldosterone and catecholamines from the interrenal gland of Triturus carnifex in response to adrenocorticotropic hormone (ACTH) administration. $J$. Morphol. 262, 692-700.

Cartledge, V. A. and Jones, S. M. (2007). Does adrenal responsiveness vary with sex and reproductive status in Egernia whitii, a viviparous skink? Gen. Comp. Endocrinol. $150,132-139$.

Cease, A. J., Lutterschmidt, D. I. and Mason, R. T. (2007). Corticosterone and the transition from courtship behavior to dispersal in male red-sided garter snakes (Thamnophis sirtalis parietalis). Gen. Comp. Endocrinol. 150, 124-131.

Clearwater, S. J. and Pankhurst, N. W. (1997). The response to capture and confinement stress of plasma cortisol, plasma sex steroids and vitellogenic oocytes in the 
marine teleost, red gurnard. J. Fish Biol. 50, 429-441.

Cockrem, J. F. and Silverin, B. (2002). Variation within and between Birds in Corticosterone Responses of Great Tits (Parus major). Gen. Comp. Endocrinol. $125,197-206$.

Coddington, E. J. and Cree, A. (1995). Effect of Acute Captivity Stress on Plasma Concentrations of Corticosterone and Sex Steroids in Female Whistling Frogs, Litoria ewingi. Gen. Comp. Endocrinol. 100, 33-38.

Crews, D. (1984). Gamete production, sex hormone secretion, and mating behavior uncoupled. Horm. Behav. 18, 22-28.

Crews, D., Grassman, M., Garstka, W. R., Halpert, A. and Camazine, B. (1987). Sex and seasonal differences in metabolism in the red-sided garter snake, Thamnophis sirtalis parietalis. Can. J. Zool. 65, 2362-2368.

Dayger, C. A., Cease, A. J. and Lutterschmidt, D. I. (2013). Responses to capture stress and exogenous corticosterone vary with body condition in female red-sided garter snakes (Thamnophis sirtalis parietalis). Horm. Behav. 64, 748-754.

Duckworth, R. A., Mendonça, M. T. and Hill, G. E. (2001). A condition dependent link between testosterone and disease resistance in the house finch. Proc. R. Soc. Lond. B Biol. Sci. 268, 2467-2472.

Elsey, R. M., Lance, V. A., Joanen, T. and Mcnease, L. (1991). Acute stress suppresses plasma estradiol levels in female alligators (Alligator mississippiensis). Comp. Biochem. Physiol. A Physiol. 100, 649-651.

Emerson, S. B. (2001). Male advertisement calls: behavioral variation and physiological processes. Anuran Commun. 36-44.

Friesen, C. R., Mason, R. T., Arnold, S. J. and Estes, S. (2013). Patterns of sperm use in two populations of Red-sided Garter Snake (Thamnophis sirtalis parietalis) with long-term female sperm storage. Can. J. Zool. 92, 33-40.

Garstka, W. R., Camazine, B. and Crews, D. (1982). Interactions of behavior and physiology during the annual reproductive cycle of the red-sided garter snake (Thamnophis sirtalis parietalis). Herpetologica 104-123.

Garstka, W. R., Tokarz, R. R., Diamond, M., Halpert, A. and Crews, D. (1985). Behavioral and physiological control of yolk synthesis and deposition in the female red-sided garter snake (Thamnophis sirtalis parietalis). Horm. Behav. 19, $137-153$.

Girard, C., Brodeur, J. C. and Hontela, A. (1998). Responsiveness of the interrenal tissue of yellow perch (Perca flavescens) from contaminated sites to an ACTH challenge test in vivo. Can. J. Fish. Aquat. Sci. 55, 438-450.

Gist, D. H. (1972). Effects of mammalian ACTH on liver and muscle glycogen levels in the South American caiman (Caiman sclerops). Gen. Comp. Endocrinol. 19, 1-6.

Gregory, P. T. (1977). Life-history parameters of the red-sided garter snake (Thamnophis sirtalis parietalis) in an extreme environment, the Interlake Region of Manitoba. Ottawa: National Museums of Canada.

Gregory, P. T. and Stewart, K. W. (1975). Long-distance dispersal and feeding strategy of the red-sided garter snake (Thamnophis sirtalis parietalis) in the Interlake of Manitoba. Can. J. Zool. 53, 238-245. 
Halpert, A. P., Garstka, W. R. and Crews, D. (1982). Sperm transport and storage and its relation to the annual sexual cycle of the female red-sided garter snake (Thamnophis sirtalis parietalis). J. Morphol. 174, 149-159.

Hau, M., Ricklefs, R. E., Wikelski, M., Lee, K. A. and Brawn, J. D. (2010). Corticosterone, testosterone and life-history strategies of birds. Proc. R. Soc. B Biol. Sci. 277, 3203-3212.

Heath, J. A. and Dufty, J. (1998). Body condition and the adrenal stress response in captive American kestrel juveniles. Physiol. Biochem. Zool. 71, 67-73.

Ingram, Crockford, J. N. and Matthews, L. R. (1999). Ultradian, circadian and seasonal rhythms in cortisol secretion and adrenal responsiveness to ACTH and yarding in unrestrained red deer (Cervus elaphus) stags. J. Endocrinol. 162, 289-300.

Jessop, T. S., Sumner, J. M., Limpus, C. J. and Whittier, J. M. (2004). Interplay between plasma hormone profiles, sex and body condition in immature hawksbill turtles (Eretmochelys imbricata) subjected to a capture stress protocol. Comp. Biochem. Physiol. A. Mol. Integr. Physiol. 137, 197-204.

Keenan, D. M., Roelfsema, F., Carroll, B. J., Iranmanesh, A. and Veldhuis, J. D. (2009). Sex defines the age dependence of endogenous ACTH-cortisol dose responsiveness. Am. J. Physiol. - Regul. Integr. Comp. Physiol. 297, R515-R523.

Klukowski, M. (2011). Effects of breeding season, testosterone and ACTH on the corticosterone response of free-ranging male fence lizards (Sceloporus undulatus). Gen. Comp. Endocrinol. 173, 295-302.

Knapp, R. and Moore, M. C. (1997). Male Morphs in Tree Lizards Have Different Testosterone Responses to Elevated Levels of Corticosterone. Gen. Comp. Endocrinol. 107, 273-279.

Krohmer, R. W., Grassman, M. and Crews, D. (1987). Annual reproductive cycle in the male red-sided garter snake, Thamnophis sirtalis parietalis: field and laboratory studies. Gen. Comp. Endocrinol. 68, 64-75.

Landys, M. M., Ramenofsky, M. and Wingfield, J. C. (2006). Actions of glucocorticoids at a seasonal baseline as compared to stress-related levels in the regulation of periodic life processes. Gen. Comp. Endocrinol. 148, 132-149.

Leary, C. J. and Harris, S. (2012). Steroid hormone levels in calling males and males practicing alternative non-calling mating tactics in the green treefrog, $\mathrm{Hyla}$ cinerea. Horm. Behav.

Leary, C. J., Jessop, T. S., Garcia, A. M. and Knapp, R. (2004). Steroid hormone profiles and relative body condition of calling and satellite toads: implications for proximate regulation of behavior in anurans. Behav. Ecol. 15, 313-320.

Lutterschmidt, D. I. (2012). Chronobiology of reproduction in garter snakes: Neuroendocrine mechanisms and geographic variation. Gen. Comp. Endocrinol. $176,448-455$.

Lutterschmidt, D. I. and Maine, A. R. (2014). Sex or candy? Neuroendocrine regulation of the seasonal transition from courtship to feeding behavior in male red-sided garter snakes (Thamnophis sirtalis parietalis). Horm. Behav. 66, 120-134.

Lutterschmidt, D. I. and Mason, R. T. (2005). A serotonin receptor antagonist, but not melatonin, modulates hormonal responses to capture stress in two populations of 
garter snakes (Thamnophis sirtalis parietalis and Thamnophis sirtalis concinnus). Gen. Comp. Endocrinol. 141, 259-270.

Lutterschmidt, D. I. and Mason, R. T. (2008). Geographic variation in timekeeping systems among three populations of garter snakes (Thamnophis sirtalis) in a common garden. Physiol. Biochem. Zool. PBZ 81, 810-825.

Lutterschmidt, D. I. and Mason, R. T. (2009). Endocrine mechanisms mediating temperature-induced reproductive behavior in red-sided garter snakes (Thamnophis sirtalis parietalis). J. Exp. Biol. 212, 3108-3118.

Lutterschmidt, D. I. and Mason, R. T. (2010). Temporally distinct effects of stress and corticosterone on diel melatonin rhythms of red-sided garter snakes (Thamnophis sirtalis). Gen. Comp. Endocrinol. 169, 11-17.

Lutterschmidt, D. I., LeMaster, M. P. and Mason, R. T. (2004). Effects of melatonin on the behavioral and hormonal responses of red-sided garter snakes (Thamnophis sirtalis parietalis) to exogenous corticosterone. Horm. Behav. 46, 692-702.

Lutterschmidt, W. I., Lutterschmidt, D. I., Mason, R. T. and Reinert, H. K. (2009). Seasonal variation in hormonal responses of timber rattlesnakes (Crotalus horridus) to reproductive and environmental stressors. J. Comp. Physiol. [B] 179, 747-757.

Mahmoud, I. Y., Guillette Jr, L. J., McAsey, M. E. and Cady, C. (1989). Stress-induced changes in serum testosterone, estradiol-17 $\beta$ and progesterone in the turtle, Chelydra serpentina. Comp. Biochem. Physiol. A Physiol. 93, 423-427.

Mashburn, K. L. and Atkinson, S. (2008). Variability in leptin and adrenal response in juvenile Steller sea lions (Eumetopias jubatus) to adrenocorticotropic hormone (ACTH) in different seasons. Gen. Comp. Endocrinol. 155, 352-358.

Moore, I. T. and Jessop, T. S. (2003). Stress, reproduction, and adrenocortical modulation in amphibians and reptiles. Horm. Behav. 43, 39-47.

Moore, I. T. and Mason, R. T. (2001). Behavioral and hormonal responses to corticosterone in the male red-sided garter snake, Thamnophis sirtalis parietalis. Physiol. Behav. 72, 669-674.

Moore, M. C., Thompson, C. W. and Marler, C. A. (1991). Reciprocal changes in corticosterone and testosterone levels following acute and chronic handling stress in the tree lizard, Urosaurus ornatus. Gen. Comp. Endocrinol. 81, 217-226.

Moore, I. T., LeMaster, M. P. and Mason, R. T. (2000a). Behavioural and hormonal responses to capture stress in the male red-sided garter snake, Thamnophis sirtalis parietalis. Anim. Behav. 59, 529-534.

Moore, I. T., Lerner, J. P., Lerner, D. T. and Mason, R. T. (2000b). Relationships between annual cycles of testosterone, corticosterone, and body condition in male red-spotted garter snakes, Thamnophis sirtalis concinnus. Physiol. Biochem. Zool. PBZ 73, 307-312.

Moore, I. T., Greene, M. J. and Mason, R. T. (2001). Environmental and seasonal adaptations of the adrenocortical and gonadal responses to capture stress in two populations of the male garter snake, Thamnophis sirtalis. J. Exp. Zool. 289, 99108.

O’Donnell, R. P., Shine, R. and Mason, R. T. (2004). Seasonal Anorexia in the Male 
Red-Sided Garter Snake, Thamnophis sirtalis parietalis. Behav. Ecol. Sociobiol. 56, 413-419.

Phillips, J. B. and Klukowski, M. (2008). Influence of season and adrenocorticotropic hormone on corticosterone in free-living female Eastern Fence Lizards (Sceloporus undulatus). J. Inf. 2008,.

Pottinger, T. G., Henrys, P. A., Williams, R. J. and Matthiessen, P. (2013). The stress response of three-spined sticklebacks is modified in proportion to effluent exposure downstream of wastewater treatment works. Aquat. Toxicol. Amst. Neth. $126,382-392$.

Rensel, M. A. and Schoech, S. J. (2011). Repeatability of baseline and stress-induced corticosterone levels across early life stages in the Florida scrub-jay (Aphelocoma coerulescens). Horm. Behav. 59, 497-502.

Rivier, C. and Rivest, S. (1991). Effect of stress on the activity of the hypothalamicpituitary-gonadal axis: peripheral and central mechanisms. Biol. Reprod. 45, 523532.

Romero, L. M. (2002). Seasonal changes in plasma glucocorticoid concentrations in freeliving vertebrates. Gen. Comp. Endocrinol. 128, 1-24.

Romero, L. M. and Wikelski, M. (2006). Diurnal and nocturnal differences in hypothalamic-pituitary-adrenal axis function in Galápagos marine iguanas. Gen. Comp. Endocrinol. 145, 177-181.

Romero, L. M. and Wingfield, J. C. (1998). Seasonal Changes in Adrenal Sensitivity Alter Corticosterone Levels in Gambel's White-Crowned Sparrows (Zonotrichia leucophrys gambelii). Comp. Biochem. Physiol. C Pharmacol. Toxicol. Endocrinol. 119, 31-36.

Romero, L. M. and Wingfield, J. C. (1999). Alterations in hypothalamic-pituitary-adrenal function associated with captivity in Gambel's white-crowned sparrows (Zonotrichia leucophrys gambelii). Comp. Biochem. Physiol. B Biochem. Mol. Biol. 122, 13-20.

Romero, L. M. and Wingfield, J. C. (2001). Regulation of the hypothalamic-pituitaryadrenal axis in free-living pigeons. J. Comp. Physiol. B 171, 231-235.

Romero, L. M., Soma, K. K. and Wingfield, J. C. (1998). Changes in pituitary and adrenal sensitivities allow the snow bunting (Plectrophenax nivalis), an Arcticbreeding song bird, to modulate corticosterone release seasonally. J. Comp. Physiol. B 168, 353-358.

Sheskin, D. J. (2007). Handbook of Parametric and Nonparametric Statistical Procedures. Boca Raton, FL: Chapman \& Hall.

Shine, R. and Mason, R. T. (2005). Do a male garter snake's energy stores limit his reproductive effort? Can. J. Zool. 83, 1265-1270.

Shine, R., Elphick, M. J., Harlow, P. S., Moore, I. T., LeMaster, M. P. and Mason, R. T. (2001). Movements, Mating, and Dispersal of Red-Sided Gartersnakes (Thamnophis sirtalis parietalis) from a Communal Den in Manitoba. Copeia 2001, 82-91.

Shors, T. J., Pickett, J., Wood, G. and Paczynski, M. (1999). Acute Stress Persistently Enhances Estrogen Levels in the Female Rat. Stress 3, 163-171. 
Uhrig, E. J., Lutterschmidt, D. I., Mason, R. T. and LeMaster, M. P. (2012). Pheromonal Mediation of Intraseasonal Declines in the Attractivity of Female Red-Sided Garter Snakes, Thamnophis sirtalis parietalis. J. Chem. Ecol.

van Lier, E., Pérez-Clariget, R. and Forsberg, M. (2003). Sex differences in cortisol secretion after administration of an ACTH analogue in sheep during the breeding and non-breeding season. Anim. Reprod. Sci. 79, 81-92.

Whittier, J. M., Mason, R. T. and Crews, D. (1987). Plasma steroid hormone levels of female red-sided garter snakes, Thamnophis sirtalis parietalis: relationship to mating and gestation. Gen. Comp. Endocrinol. 67, 33-43.

Wingfield, J. C. (2008). Comparative endocrinology, environment and global change. Gen. Comp. Endocrinol. 157, 207-216.

Wingfield, J. C. and Sapolsky, R. M. (2003). Reproduction and Resistance to Stress: When and How. J. Neuroendocrinol. 15, 711-724.

Wingfield, J. C., Deviche, P., Sharbaugh, S., Astheimer, L. B., Holberton, R., Suydam, R. and Hunt, K. (1994). Seasonal changes of the adrenocortical responses to stress in redpolls, Acanthis flammea, in Alaska. J. Exp. Zool. 270, 372-380.

Woodley, S. K. and Moore, M. C. (2002). Plasma corticosterone response to an acute stressor varies according to reproductive condition in female tree lizards (Urosaurus ornatus). Gen. Comp. Endocrinol. 128, 143-148.

Zar, J. H. (1999). Biostatistical analysis. Prentice hall Upper Saddle River, NJ.

Zerani, M., Amabili, F., Mosconi, G. and Gobbetti, A. (1991). Effects of captivity stress on plasma steroid levels in the green frog, Rana esculenta, during the annual reproductive cycle. Comp. Biochem. Physiol. A Physiol. 98, 491-496.

\section{Chapter 3 References}

Anderson, L., Cree, A., Towns, D., Nelson, N., 2014. Modulation of corticosterone secretion in tuatara (Sphenodon punctatus): Evidence of a dampened stress response in gravid females. Gen. Comp. Endocrinol. 201, 45-52. doi:10.1016/j.ygcen.2014.03.035

Armitage, K.B., 1991. Factors affecting corticosteroid concentrations in yellow-bellied marmots. Comp. Biochem. Physiol. A Physiol. 98, 47-54. doi:10.1016/03009629(91)90576-X

Astheimer, L.B., Buttemer, W.A., Wingfield, J.C., 1995. Seasonal and acute changes in adrenocortical responsiveness in an arctic-breeding bird. Horm. Behav. 29, 442457. doi:10.1006/hbeh.1995.1276

Ball, G.F., Ketterson, E.D., 2008. Sex differences in the response to environmental cues regulating seasonal reproduction in birds. Philos. Trans. R. Soc. B Biol. Sci. 363, 231-246. doi:10.1098/rstb.2007.2137

Bauer, C.M., Hayes, L.D., Ebensperger, L.A., Romero, L.M., 2014. Seasonal variation in the degu (Octodon degus) endocrine stress response. Gen. Comp. Endocrinol. 197, 26-32. doi:10.1016/j.ygcen.2013.11.025

Brann, D.W., Mahesh, V.B., 1991. Role of corticosteroids in female reproduction. 
FASEB J. 5, 2691-2698.

Breuner, C.W., Orchinik, M., 2001. Seasonal regulation of membrane and intracellular corticosteroid receptors in the house sparrow brain. J. Neuroendocrinol. 13, 412420.

Capaldo, A., Gay, F., Valiante, S., Varlese, M. g., Laforgia, V., Varano, L., 2004. Release of aldosterone and catecholamines from the interrenal gland of Triturus carnifex in response to adrenocorticotropic hormone (ACTH) administration. J. Morphol. 262, 692-700. doi:10.1002/jmor.10269

Cartledge, V.A., Jones, S.M., 2007. Does adrenal responsiveness vary with sex and reproductive status in Egernia whitii, a viviparous skink? Gen. Comp. Endocrinol. 150, 132-139. doi:10.1016/j.ygcen.2006.07.021

Cease, A.J., Lutterschmidt, D.I., Mason, R.T., 2007. Corticosterone and the transition from courtship behavior to dispersal in male red-sided garter snakes (Thamnophis sirtalis parietalis). Gen. Comp. Endocrinol. 150, 124-131.

Cockrem, J.F., Silverin, B., 2002. Variation within and between Birds in Corticosterone Responses of Great Tits (Parus major). Gen. Comp. Endocrinol. 125, 197-206.

Dayger, C.A., Cease, A.J., Lutterschmidt, D.I., 2013. Responses to capture stress and exogenous corticosterone vary with body condition in female red-sided garter snakes (Thamnophis sirtalis parietalis). Horm. Behav. 64, 748-754. doi:10.1016/j.yhbeh.2013.09.003

Dayger, C.A., Lutterschmidt, D.I., 2016. Seasonal and sex differences in responsiveness to adrenocorticotropic hormone contribute to stress response plasticity in redsided garter snakes (Thamnophis sirtalis parietalis). J. Exp. Biol. jeb.130450. doi: $10.1242 /$ jeb. 130450

Eikenaar, C., Müller, F., Klinner, T., Bairlein, F., 2015. Baseline corticosterone levels are higher in migrating than sedentary common blackbirds in autumn, but not in spring. Gen. Comp. Endocrinol. 224, 121-125. doi:10.1016/j.ygcen.2015.07.003

Fanson, K.V., Parrott, M.L., 2015. The value of eutherian-marsupial comparisons for understanding the function of glucocorticoids in female mammal reproduction. Horm. Behav., Annual Conference SBN 2014 76, 41-47. doi:10.1016/j.yhbeh.2015.05.012

Girard, C., Brodeur, J.C., Hontela, A., 1998. Responsiveness of the interrenal tissue of yellow perch (Perca flavescens) from contaminated sites to an ACTH challenge test in vivo. Can. J. Fish. Aquat. Sci. 55, 438-450. doi:10.1139/f97-224

Gist, D.H., 1972. Effects of mammalian ACTH on liver and muscle glycogen levels in the South American caiman (Caiman sclerops). Gen. Comp. Endocrinol. 19, 1-6. doi:10.1016/0016-6480(72)90001-9

Gregory, P.T., Stewart, K.W., 1975. Long-distance dispersal and feeding strategy of the red-sided garter snake (Thamnophis sirtalis parietalis) in the Interlake of Manitoba. Can. J. Zool. 53, 238-245. doi:10.1139/z75-030

Gustafson, A.W., Belt, W.D., 1981. The adrenal cortex during activity and hibernation in the male little brown bat, Myotis lucifugus lucifugus: Annual rhythm of plasma cortisol levels. Gen. Comp. Endocrinol. 44, 269-278. doi:10.1016/00166480(81)90001-0 
Heath, J.A., Dufty, J., 1998. Body condition and the adrenal stress response in captive American kestrel juveniles. Physiol. Biochem. Zool. 71, 67-73.

Holberton, R.L., 1999. Changes in Patterns of Corticosterone Secretion Concurrent with Migratory Fattening in a Neotropical Migratory Bird. Gen. Comp. Endocrinol. 116, 49-58. doi:10.1006/gcen.1999.7336

Holberton, R.L., Parrish, J.D., Wingfield, J.C., 1996. Modulation of the Adrenocortical Stress Response in Neotropical Migrants during Autumn Migration. The Auk 113, 558-564. doi:10.2307/4088976

Ingram, Crockford, J.N., Matthews, L.R., 1999. Ultradian, circadian and seasonal rhythms in cortisol secretion and adrenal responsiveness to ACTH and yarding in unrestrained red deer (Cervus elaphus) stags. J. Endocrinol. 162, 289-300. doi:10.1677/joe.0.1620289

Karten, Y.J.G., Olariu, A., Cameron, H.A., 2005. Stress in early life inhibits neurogenesis in adulthood. Trends Neurosci. 28, 171-172. doi:10.1016/j.tins.2005.01.009

Kitaysky, A.S., Wingfield, J.C., Piatt, J.F., 1999. Dynamics of food availability, body condition and physiological stress response in breeding Black-legged Kittiwakes. Funct. Ecol. 13, 577-584. doi:10.1046/j.1365-2435.1999.00352.x

Klukowski, M., 2011. Effects of breeding season, testosterone and ACTH on the corticosterone response of free-ranging male fence lizards (Sceloporus undulatus). Gen. Comp. Endocrinol. 173, 295-302. doi:10.1016/j.ygcen.2011.06.006

Landys, M.M., Ramenofsky, M., Wingfield, J.C., 2006. Actions of glucocorticoids at a seasonal baseline as compared to stress-related levels in the regulation of periodic life processes. Gen. Comp. Endocrinol. 148, 132-149. doi:10.1016/j.ygcen.2006.02.013

Lattin, C.R., Bauer, C.M., de Bruijn, R., Michael Romero, L., 2012a. Hypothalamuspituitary-adrenal axis activity and the subsequent response to chronic stress differ depending upon life-history stage. Gen. Comp. Endocrinol. 178, 494-501. doi:10.1016/j.ygcen.2012.07.013

Lattin, C.R., Breuner, C.W., Michael Romero, L., 2016. Does corticosterone regulate the onset of breeding in free-living birds?: The CORT-Flexibility Hypothesis and six potential mechanisms for priming corticosteroid function. Horm. Behav. 78, 107120. doi:10.1016/j.yhbeh.2015.10.020

Lattin, C.R., Waldron-Francis, K., Richardson, J.W., de Bruijn, R., Bauer, C.M., Breuner, C.W., Michael Romero, L., 2012b. Pharmacological characterization of intracellular glucocorticoid receptors in nine tissues from house sparrow (Passer domesticus). Gen. Comp. Endocrinol. 179, 214-220. doi:10.1016/j.ygcen.2012.08.007

Liebl, A.L., Shimizu, T., Martin, L.B., 2013. Covariation among glucocorticoid regulatory elements varies seasonally in house sparrows. Gen. Comp. Endocrinol. 183C, 32-37. doi:10.1016/j.ygcen.2012.11.021

Lutterschmidt, D.I., Mason, R.T., 2010. Temporally distinct effects of stress and corticosterone on diel melatonin rhythms of red-sided garter snakes (Thamnophis sirtalis). Gen. Comp. Endocrinol. 169, 11-17. doi:10.1016/j.ygcen.2010.06.013 
Lutterschmidt, D.I., Mason, R.T., 2009. Endocrine mechanisms mediating temperatureinduced reproductive behavior in red-sided garter snakes (Thamnophis sirtalis parietalis). J. Exp. Biol. 212, 3108-3118. doi:10.1242/jeb.033100

Lutterschmidt, D.I., Mason, R.T., 2005. A serotonin receptor antagonist, but not melatonin, modulates hormonal responses to capture stress in two populations of garter snakes (Thamnophis sirtalis parietalis and Thamnophis sirtalis concinnus). Gen. Comp. Endocrinol. 141, 259-270. doi:10.1016/j.ygcen.2005.01.012

Lutterschmidt, W.I., Lutterschmidt, D.I., Mason, R.T., Reinert, H.K., 2009. Seasonal variation in hormonal responses of timber rattlesnakes (Crotalus horridus) to reproductive and environmental stressors. J. Comp. Physiol. [B] 179, 747-757. doi:10.1007/s00360-009-0356-2

Mashburn, K.L., Atkinson, S., 2008. Variability in leptin and adrenal response in juvenile Steller sea lions (Eumetopias jubatus) to adrenocorticotropic hormone (ACTH) in different seasons. Gen. Comp. Endocrinol. 155, 352-358. doi:10.1016/j.ygcen.2007.05.030

Moore, I.T., Greene, M.J., Mason, R.T., 2001. Environmental and seasonal adaptations of the adrenocortical and gonadal responses to capture stress in two populations of the male garter snake, Thamnophis sirtalis. J. Exp. Zool. 289, 99-108.

Moore, I.T., LeMaster, M.P., Mason, R.T., 2000. Behavioural and hormonal responses to capture stress in the male red-sided garter snake, Thamnophis sirtalis parietalis. Anim. Behav. 59, 529-534. doi:10.1006/anbe.1999.1344

Muráni, E., Ponsuksili, S., D’Eath, R.B., Turner, S.P., Kurt, E., Evans, G., Thölking, L., Klont, R., Foury, A., Mormède, P., Wimmers, K., 2010. Association of HPA axisrelated genetic variation with stress reactivity and aggressive behaviour in pigs. BMC Genet. 11, 74. doi:10.1186/1471-2156-11-74

Owen, D.A.S., Carter, E.T., Holding, M.L., Islam, K., Moore, I.T., 2014. Roads are associated with a blunted stress response in a North American pit viper. Gen. Comp. Endocrinol. 202, 87-92. doi:10.1016/j.ygcen.2014.04.020

Pottinger, T.G., Henrys, P.A., Williams, R.J., Matthiessen, P., 2013. The stress response of three-spined sticklebacks is modified in proportion to effluent exposure downstream of wastewater treatment works. Aquat. Toxicol. Amst. Neth. 126, 382-392. doi:10.1016/j.aquatox.2012.09.002

Raja-aho, S., Lehikoinen, E., Suorsa, P., Nikinmaa, M., Vainio, M., Vosloo, D., Eeva, T., 2013. Corticosterone secretion patterns prior to spring and autumn migration differ in free-living barn swallows (Hirundo rustica L.). Oecologia 173, 689-697. doi:10.1007/s00442-013-2669-9

Reeder, D.M., Kosteczko, N.S., Kunz, T.H., Widmaier, E.P., 2004. Changes in baseline and stress-induced glucocorticoid levels during the active period in free-ranging male and female little brown myotis, Myotis lucifugus (Chiroptera: Vespertilionidae). Gen. Comp. Endocrinol. 136, 260-269. doi:10.1016/j.ygcen.2003.12.020

Rensel, M.A., Schoech, S.J., 2011. Repeatability of baseline and stress-induced corticosterone levels across early life stages in the Florida scrub-jay (Aphelocoma coerulescens). Horm. Behav. 59, 497-502. doi:10.1016/j.yhbeh.2011.01.010 
Romero, L.M., 2002. Seasonal changes in plasma glucocorticoid concentrations in freeliving vertebrates. Gen. Comp. Endocrinol. 128, 1-24. doi:10.1016/S00166480(02)00064-3

Romero, L.M., Ramenofsky, M., Wingfield, J.C., 1997. Season and Migration Alters the Corticosterone Response to Capture and Handling in an Arctic Migrant, the White-Crowned Sparrow (Zonotrichia leucophrys gambelii). Comp. Biochem. Physiol. C Pharmacol. Toxicol. Endocrinol. 116, 171-177. doi:10.1016/S07428413(96)00208-3

Romero, L.M., Soma, K.K., Wingfield, J.C., 1998. Changes in pituitary and adrenal sensitivities allow the snow bunting (Plectrophenax nivalis), an Arctic-breeding song bird, to modulate corticosterone release seasonally. J. Comp. Physiol. B 168, 353-358. doi:10.1007/s003600050154

Romero, L.M., Wikelski, M., 2010. Stress physiology as a predictor of survival in Galapagos marine iguanas. Proc. R. Soc. B Biol. Sci. 277, 3157-3162. doi:10.1098/rspb.2010.0678

Romero, L.M., Wingfield, J.C., 1998. Seasonal Changes in Adrenal Sensitivity Alter Corticosterone Levels in Gambel's White-Crowned Sparrows (Zonotrichia leucophrys gambelii). Comp. Biochem. Physiol. C Pharmacol. Toxicol. Endocrinol. 119, 31-36. doi:10.1016/S0742-8413(97)00167-9

Sapolsky, R.M., Romero, L.M., Munck, A.U., 2000. How do glucocorticoids influence stress responses? Integrating permissive, suppressive, stimulatory, and preparative actions 1. Endocr. Rev. 21, 55-89.

Schiml, P.A., Rissman, E.F., 1999. Cortisol facilitates induction of sexual behavior in the female musk shrew (Suncus murinus). Behav. Neurosci. 113, 166-175. doi:10.1037/0735-7044.113.1.166

Schmidt, K.L., Furlonger, A.A., Lapierre, J.M., MacDougall-Shackleton, E.A., MacDougall-Shackleton, S.A., 2012. Regulation of the HPA axis is related to song complexity and measures of phenotypic quality in song sparrows. Horm. Behav. 61, 652-659. doi:10.1016/j.yhbeh.2012.02.027

Schoech, S.J., Mumme, R.L., Wingfield, J.C., 1997. Corticosterone, Reproductive Status, and Body Mass in a Cooperative Breeder, the Florida Scrub-Jay (Aphelocoma coerulescens). Physiol. Zool. 70, 68-73. doi:10.2307/30164285

Schwartz, T.S., Bronikowski, A.M., 2013. Dissecting molecular stress networks: identifying nodes of divergence between life-history phenotypes. Mol. Ecol. 22, 739-756. doi:10.1111/j.1365-294X.2012.05750.x

Shine, R., Olsson, M.M., Mason, R.T., 2000. Chastity belts in gartersnakes: the functional significance of mating plugs. Biol. J. Linn. Soc. 70, 377-390. doi:10.1111/j.1095-8312.2000.tb01229.x

Shivatcheva, T.M., Ankov, V.K., Hadjioloff, A.I., 1988. Circannual fluctuations of the serum cortisol in the european ground squirrel, Citellus citellus L. Comp. Biochem. Physiol. A Physiol. 90, 515-518. doi:10.1016/0300-9629(88)90229-0

van Lier, E., Pérez-Clariget, R., Forsberg, M., 2003. Sex differences in cortisol secretion after administration of an ACTH analogue in sheep during the breeding and nonbreeding season. Anim. Reprod. Sci. 79, 81-92. doi:10.1016/S0378- 
4320(03)00083-6

Walker, B.G., Meddle, S.L., Romero, L.M., Landys, M.M., Reneerkens, J., Wingfield, J.C., 2015. Breeding on the extreme edge: Modulation of the adrenocortical response to acute stress in two High Arctic passerines. J. Exp. Zool. Part Ecol. Genet. Physiol. 323, 266-275. doi:10.1002/jez.1923

Whitnall, M.H., 1993. Regulation of the hypothalamic corticotropin-releasing hormone neurosecretory system. Prog. Neurobiol. 40, 573-629. doi:10.1016/03010082(93)90035-Q

Whittier, J.M., Mason, R.T., Crews, D., 1987. Plasma steroid hormone levels of female red-sided garter snakes, Thamnophis sirtalis parietalis: relationship to mating and gestation. Gen. Comp. Endocrinol. 67, 33-43.

Wingfield, J.C., Deviche, P., Sharbaugh, S., Astheimer, L.B., Holberton, R., Suydam, R., Hunt, K., 1994. Seasonal changes of the adrenocortical responses to stress in redpolls, Acanthis flammea, in Alaska. J. Exp. Zool. 270, 372-380. doi:10.1002/jez.1402700406

\section{Chapter 4 References}

Astheimer, L.B., Buttemer, W.A., Wingfield, J.C., 1995. Seasonal and acute changes in adrenocortical responsiveness in an arctic-breeding bird. Horm. Behav. 29, 442457.

Bonnet, X., Bradshaw, D., Shine, R., 1998. Capital versus Income Breeding: An Ectothermic Perspective. Oikos 83, 333-342.

Breuner, C.W., Orchinik, M., 2002. Plasma binding proteins as mediators of corticosteroid action in vertebrates. J. Endocrinol. 175, 99-112.

Cartledge, V.A., Jones, S.M., 2007. Does adrenal responsiveness vary with sex and reproductive status in Egernia whitii, a viviparous skink? Gen. Comp. Endocrinol. $150,132-139$.

Cease, A.J., Lutterschmidt, D.I., Mason, R.T., 2007. Corticosterone and the transition from courtship behavior to dispersal in male red-sided garter snakes (Thamnophis sirtalis parietalis). Gen. Comp. Endocrinol. 150, 124-131.

Charland, M.B., Gregory, P.T., 1995. Movements and habitat use in gravid and nongravid female garter snakes (Colubridae: Thamnophis). J. Zool. 236, 543561.

Clearwater, S., Pankhurst, N., 1997. The response to capture and confinement stress of plasma cortisol, plasma sex steroids and vitellogenic oocytes in the marine teleost, red gurnard. J. Fish Biol. 50, 429.

Cleary, J.J., Battaglene, S.C., Pankhurst, N.W., 2002. Capture and handling stress affects the endocrine and ovulatory response to exogenous hormone treatment in snapper, Pagrus auratus (Bloch \& Schneider). Aquac. Res. 33, 829-838.

Coddington, E.J., Cree, A., 1995. Effect of Acute Captivity Stress on Plasma Concentrations of Corticosterone and Sex Steroids in Female Whistling Frogs, Litoria ewingi. Gen. Comp. Endocrinol. 100, 33-38. 
Cook, K.V., O’Connor, C.M., Gilmour, K.M., Cooke, S.J., 2011. The glucocorticoid stress response is repeatable between years in a wild teleost fish. J. Comp. Physiol. 197, 1189-1196.

Crews, D., 1984. Gamete production, sex hormone secretion, and mating behavior uncoupled. Horm. Behav. 18, 22-28.

Drent, R.H., Daan, S., 1980. The prudent parent: energetic adjustments in avian breeding. Ardea 68, 225-252.

Garstka, W.R., Camazine, B., Crews, D., 1982. Interactions of behavior and physiology during the annual reproductive cycle of the red-sided garter snake (Thamnophis sirtalis parietalis). Herpetologica 104-123.

Ghisleni, G., Capiotti, K.M., Da Silva, R.S., Oses, J.P., Piato, Â.L., Soares, V., Bogo, M.R., Bonan, C.D., 2012. The role of CRH in behavioral responses to acute restraint stress in zebrafish. Prog. Neuropsychopharmacol. Biol. Psychiatry 36, 176-182.

Gregory, P.T., 1977. Life-history parameters of the red-sided garter snake (Thamnophis sirtalis parietalis) in an extreme environment, the Interlake Region of Manitoba. National Museums of Canada, Ottawa.

Gregory, P.T., Crampton, L.H., Skebo, K.M., 1999. Conflicts and interactions among reproduction, thermoregulation and feeding in viviparous reptiles: are gravid snakes anorexic? J. Zool. 248, 231-241.

Harper, J.M., Austad, S.N., 2001. Effect of capture and season on fecal glucocorticoid levels in deer mice (Peromyscus maniculatus) and red-backed voles (Clethrionomys gapperi). Gen. Comp. Endocrinol. 123, 337-344.

Harvey, S., Phillips, J.G., Rees, A., Hall, T.R., 2005. Stress and adrenal function. J. Exp. Zool. 232, 633-645.

Heath, J.A., Dufty, J., 1998. Body condition and the adrenal stress response in captive American kestrel juveniles. Physiol. Biochem. Zool. 71, 67-73.

Heino, Kaitala, 1999. Evolution of resource allocation between growth and reproduction in animals with indeterminate growth. J. Evol. Biol. 12, 423-429.

Joy, J.E., Crews, D., 1985. Social dynamics of group courtship behavior in male redsided garter snakes (Thamnophis sirtalis parietalis). J. Comp. Psychol. Wash. Dc 1983 99, 145-149.

Kitaysky, A.S., Wingfield, J.C., Piatt, J.F., 1999. Dynamics of food availability, body condition and physiological stress response in breeding Black-legged Kittiwakes. Funct. Ecol. 13, 577-584.

Kozłowski, J., 1992. Optimal allocation of resources to growth and reproduction: Implications for age and size at maturity. Trends Ecol. Evol. 7, 15-19.

Krohmer, R.W., Lutterschmidt, D.I., 2011. Environmental and neuroendocrine control of reproduction in snakes, in: Aldridge, R.D., Seaver, D.M. (Eds.), Reproductive Biology and Phylogeny of Snakes. pp. 289-346.

Lancaster, L.T., Hazard, L.C., Clobert, J., Sinervo, B.R., 2008. Corticosterone manipulation reveals differences in hierarchical organization of multidimensional reproductive trade-offs in r-strategist and K-strategist females. J. Evol. Biol. 21, $556-565$. 
Landys, M.M., Ramenofsky, M., Wingfield, J.C., 2006. Actions of glucocorticoids at a seasonal baseline as compared to stress-related levels in the regulation of periodic life processes. Gen. Comp. Endocrinol. 148, 132-149.

Lutterschmidt, D.I., LeMaster, M.P., Mason, R.T., 2004. Effects of melatonin on the behavioral and hormonal responses of red-sided garter snakes (Thamnophis sirtalis parietalis) to exogenous corticosterone. Horm. Behav. 46, 692-702.

Lutterschmidt, D.I., Mason, R.T., 2005. A serotonin receptor antagonist, but not melatonin, modulates hormonal responses to capture stress in two populations of garter snakes (Thamnophis sirtalis parietalis and Thamnophis sirtalis concinnus). Gen. Comp. Endocrinol. 141, 259-270.

Lutterschmidt, D.I., Mason, R.T., 2008. Geographic variation in timekeeping systems among three populations of garter snakes (Thamnophis sirtalis) in a common garden. Physiol. Biochem. Zool. Pbz 81, 810-825.

Lutterschmidt, D.I., Mason, R.T., 2009. Endocrine mechanisms mediating temperatureinduced reproductive behavior in red-sided garter snakes (Thamnophis sirtalis parietalis). J. Exp. Biol. 212, 3108-3118.

Lutterschmidt, D.I., Mason, R.T., 2010. Temporally distinct effects of stress and corticosterone on diel melatonin rhythms of red-sided garter snakes (Thamnophis sirtalis). Gen. Comp. Endocrinol. 169, 11-17.

Lutterschmidt, W.I., Lutterschmidt, D.I., Mason, R.T., Reinert, H.K., 2009. Seasonal variation in hormonal responses of timber rattlesnakes (Crotalus horridus) to reproductive and environmental stressors. J. Comp. Physiol. [B] 179, 747-757.

Mahmoud, I.Y., Guillette Jr, L.J., McAsey, M.E., Cady, C., 1989. Stress-induced changes in serum testosterone, estradiol-17 $\beta$ and progesterone in the turtle, Chelydra serpentina. Comp. Biochem. Physiol. A Physiol. 93, 423-427.

Manire, C.A., Rasmussen, L.E.L., Maruska, K.P., Tricas, T.C., 2007. Sex, seasonal, and stress-related variations in elasmobranch corticosterone concentrations. Comp. Biochem. Physiol. A. Mol. Integr. Physiol. 148, 926-935.

Mendonça, M.T., Crews, D., 1996. Effects of ovariectomy and estrogen replacement on attractivity and receptivity in the red-sided garter snake (Thamnophis sirtalis parietalis). J. Comp. Physiol. [A] 178, 373-381.

Minni, A.M., Dorey, R., Piérard, C., Dominguez, G., Helbling, J.-C., Foury, A., Béracochéa, D., Moisan, M.-P., 2012. Critical role of plasma corticosteroidbinding-globulin during stress to promote glucocorticoid delivery to the brain: impact on memory retrieval. Endocrinology 153, 4766-4774.

Moore, I.T., Greene, M.J., Mason, R.T., 2001. Environmental and seasonal adaptations of the adrenocortical and gonadal responses to capture stress in two populations of the male garter snake, Thamnophis sirtalis. J. Exp. Zool. 289, 99-108.

Moore, I.T., Jessop, T.S., 2003. Stress, reproduction, and adrenocortical modulation in amphibians and reptiles. Horm. Behav. 43, 39-47.

Moore, I.T., Lemaster, M.P., Mason, R.T., 2000a. Behavioural and hormonal responses to capture stress in the male red-sided garter snake, Thamnophis sirtalis parietalis. Anim. Behav. 59, 529-534.

Moore, I.T., Lerner, J.P., Lerner, D.T., Mason, R.T., 2000b. Relationships between 
annual cycles of testosterone, corticosterone, and body condition in male redspotted garter snakes, Thamnophis sirtalis concinnus. Physiol. Biochem. Zool. Pbz 73, 307-312.

Moore, I.T., Mason, R.T., 2001. Behavioral and hormonal responses to corticosterone in the male red-sided garter snake, Thamnophis sirtalis parietalis. Physiol. Behav. 72, 669-674.

Nilsson, P.B., Hollmén, T.E., Atkinson, S., Mashburn, K.L., Tuomi, P.A., Esler, D., Mulcahy, D.M., Rizzolo, D.J., 2008. Effects of ACTH, capture, and short term confinement on glucocorticoid concentrations in harlequin ducks (Histrionicus histrionicus). Comp. Biochem. Physiol. A. Mol. Integr. Physiol. 149, 275-283.

Reeder, D.M., Kosteczko, N.S., Kunz, T.H., Widmaier, E.P., 2004. Changes in baseline and stress-induced glucocorticoid levels during the active period in free-ranging male and female little brown myotis, Myotis lucifugus (Chiroptera:

Vespertilionidae). Gen. Comp. Endocrinol. 136, 260-269.

Ricciardella, L.F., Bliley, J.M., Feth, C.C., Woodley, S.K., 2010. Acute stressors increase plasma corticosterone and decrease locomotor activity in a terrestrial salamander (Desmognathus ochrophaeus). Physiol. Behav. 101, 81-86.

Romero, L.M., 2002. Seasonal changes in plasma glucocorticoid concentrations in freeliving vertebrates. Gen. Comp. Endocrinol. 128, 1-24.

Schoech, S.J., Mumme, R.L., Wingfield, J.C., 1997. Corticosterone, Reproductive Status, and Body Mass in a Cooperative Breeder, the Florida Scrub-Jay (Aphelocoma coerulescens). Physiol. Zool. 70, 68-73.

Sockman, K.W., Schwabl, H., 2001. Plasma Corticosterone in Nestling American Kestrels: Effects of Age, Handling Stress, Yolk Androgens, and Body Condition. Gen. Comp. Endocrinol. 122, 205-212.

Taylor, E.N., DeNardo, D.F., Jennings, D.H., 2004. Seasonal steroid hormone levels and their relation to reproduction in the Western Diamond-backed Rattlesnake, Crotalus atrox (Serpentes: Viperidae). Gen. Comp. Endocrinol. 136, 328-337.

Van Hout, A.J.-M., Eens, M., Darras, V.M., Pinxten, R., 2010. Acute stress induces a rapid increase of testosterone in a songbird: implications for plasma testosterone sampling. Gen. Comp. Endocrinol. 168, 505-510.

Vitousek, M.N., Mitchell, M.A., Romero, L.M., Awerman, J., Wikelski, M., 2010. To breed or not to breed: physiological correlates of reproductive status in a facultatively biennial iguanid. Horm. Behav. 57, 140-146.

Whittier, J.M., Crews, D., 1990. Body Mass and Reproduction in Female Red-Sided Garter Snakes (Thamnophis sirtalis parietalis). Herpetologica 46, 219-226.

Whittier, J.M., Mason, R.T., Crews, D., 1987. Plasma steroid hormone levels of female red-sided garter snakes, Thamnophis sirtalis parietalis: relationship to mating and gestation. Gen. Comp. Endocrinol. 67, 33-43.

Williams, C.T., Kitaysky, A.S., Kettle, A.B., Buck, C.L., 2008. Corticosterone levels of tufted puffins vary with breeding stage, body condition index, and reproductive performance. Gen. Comp. Endocrinol. 158, 29-35.

Wingfield, J., 1988. Changes in reproductive function of free-living birds in direct response to environmental perturbations. Process. Environ. Inf. Vertebr. 121-148. 
Wingfield, J., Ramenofsky, M., 1999. Hormones and the behavioral ecology of stress. Stress Physiol. Anim. 1-51.

Wingfield, J.C., Maney, D.L., Breuner, C.W., Jacobs, J.D., Lynn, S., Ramenofsky, M., Richardson, R.D., 1998. Ecological Bases of Hormone-Behavior Interactions: The "Emergency Life History Stage". Am. Zool. 38, 191-206.

Wingfield, J.C., Sapolsky, R.M., 2003. Reproduction and Resistance to Stress: When and How. J. Neuroendocrinol. 15, 711-724.

Woodley, S.K., Moore, M.C., 2002. Plasma corticosterone response to an acute stressor varies according to reproductive condition in female tree lizards (Urosaurus ornatus). Gen. Comp. Endocrinol. 128, 143-148.

Zar, J.H., 1999. Biostatistical analysis. Prentice hall Upper Saddle River, NJ.

Zerani, M., Amabili, F., Mosconi, G., Gobbetti, A., 1991. Effects of captivity stress on plasma steroid levels in the green frog, Rana esculenta, during the annual reproductive cycle. Comp. Biochem. Physiol. A Physiol. 98, 491-496.

\section{Chapter 5 References}

Anderson, L., Cree, A., Towns, D., Nelson, N., 2014. Modulation of corticosterone secretion in tuatara (Sphenodon punctatus): Evidence of a dampened stress response in gravid females. Gen. Comp. Endocrinol. 201, 45-52. doi:10.1016/j.ygcen.2014.03.035

Arora, V.K., Schenkein, E., Murali, R., Subudhi, S.K., Wongvipat, J., Balbas, M.D., Shah, N., Cai, L., Efstathiou, E., Logothetis, C., Zheng, D., Sawyers, C.L., 2013. Glucocorticoid Receptor Confers Resistance to Antiandrogens by Bypassing Androgen Receptor Blockade. Cell 155, 1309-1322. doi:10.1016/j.cell.2013.11.012

Bauer, C.M., Hayes, L.D., Ebensperger, L.A., Romero, L.M., 2014. Seasonal variation in the degu (Octodon degus) endocrine stress response. Gen. Comp. Endocrinol. 197, 26-32. doi:10.1016/j.ygcen.2013.11.025

Beach, F.A., 1976. Sexual attractivity, proceptivity, and receptivity in female mammals. Horm. Behav. 7, 105-138.

Bonnet, X., Bradshaw, D., Shine, R., 1998. Capital versus Income Breeding: An Ectothermic Perspective. Oikos 83, 333-342. doi:10.2307/3546846

Boyle, M.P., Brewer, J.A., Funatsu, M., Wozniak, D.F., Tsien, J.Z., Izumi, Y., Muglia, L.J., 2005. Acquired deficit of forebrain glucocorticoid receptor produces depression-like changes in adrenal axis regulation and behavior. Proc. Natl. Acad. Sci. U. S. A. 102, 473-478. doi:10.1073/pnas.0406458102

Bradford, M.M., 1976. A rapid and sensitive method for the quantitation of microgram quantities of protein utilizing the principle of protein-dye binding. Anal. Biochem. 72, 248-254. doi:10.1016/0003-2697(76)90527-3

Busch, D.S., Hayward, L.S., 2009. Stress in a conservation context: A discussion of glucocorticoid actions and how levels change with conservation-relevant variables. Biol. Conserv. 142, 2844-2853. doi:10.1016/j.biocon.2009.08.013 
Carpenter, C.C., 1977. Communication and Displays of Snakes. Integr Comp Biol Integr. Comp. Biol. 17, 217-223.

Cease, A.J., Lutterschmidt, D.I., Mason, R.T., 2007. Corticosterone and the transition from courtship behavior to dispersal in male red-sided garter snakes (Thamnophis sirtalis parietalis). Gen. Comp. Endocrinol. 150, 124-131.

Chiba, S., Numakawa, T., Ninomiya, M., Richards, M.C., Wakabayashi, C., Kunugi, H., 2012. Chronic restraint stress causes anxiety- and depression-like behaviors, downregulates glucocorticoid receptor expression, and attenuates glutamate release induced by brain-derived neurotrophic factor in the prefrontal cortex. Prog. Neuropsychopharmacol. Biol. Psychiatry 39, 112-119. doi:10.1016/j.pnpbp.2012.05.018

Crespi, E.J., Denver, R.J., 2004. Ontogeny of corticotropin-releasing factor effects on locomotion and foraging in the Western spadefoot toad (Spea hammondii). Horm. Behav. 46, 399-410. doi:10.1016/j.yhbeh.2004.03.011

Crews, D., 1976. Hormonal control of male courtship behavior and female attractivity in the garter snake (Thamnophis sirtalis sirtalis). Horm. Behav. 7, 451-460.

Crossin, G.T., Love, O.P., Cooke, S.J., Williams, T.D., 2016. Glucocorticoid manipulations in free-living animals: considerations of dose delivery, life-history context and reproductive state. Funct. Ecol. 30, 116-125. doi:10.1111/13652435.12482

Davis, J.R., Boyle, S.A., Khan, A.A., Gay, A.L.J., Grisham, J.M., Luque, L.E., 2012. Snake parasitism in an urban old-growth forest. Urban Ecosyst. 15, 739-752. doi: 10.1007/s11252-012-0234-7

Dayger, C.A., Cease, A.J., Lutterschmidt, D.I., 2013. Responses to capture stress and exogenous corticosterone vary with body condition in female red-sided garter snakes (Thamnophis sirtalis parietalis). Horm. Behav. 64, 748-754. doi:10.1016/j.yhbeh.2013.09.003

Dayger, C.A., Lutterschmidt, D.I., 2016. Seasonal and sex differences in responsiveness to adrenocorticotropic hormone contribute to stress response plasticity in redsided garter snakes (Thamnophis sirtalis parietalis). J. Exp. Biol. jeb.130450. doi:10.1242/jeb.130450

DiBattista, J.D., Anisman, H., Whitehead, M., Gilmour, K.M., 2005. The effects of cortisol administration on social status and brain monoaminergic activity in rainbow trout Oncorhynchus mykiss. J. Exp. Biol. 208, 2707-2718. doi:10.1242/jeb.01690

DiBattista, J.D., Levesque, H.M., Moon, T.W., Gilmour, K.M., 2006. Growth depression in socially subordinate rainbow trout Oncorhynchus mykiss: more than a fasting effect. Physiol. Biochem. Zool. PBZ 79, 675-687. doi:10.1086/504612

Dixson, A.F., Lunn, S.F., 1987. Post-partum changes in hormones and sexual behaviour in captive groups of marmosets (Callithrix jacchus). Physiol. Behav. 41, 577-583. doi:10.1016/0031-9384(87)90314-3

Drent, R.H., Daan, S., 1980. The prudent parent: energetic adjustments in avian breeding. Ardea 68, 225-252.

Dunlap, K.D., Jashari, D., Pappas, K.M., 2011. Glucocorticoid receptor blockade inhibits 
brain cell addition and aggressive signaling in electric fish, Apteronotus leptorhynchus. Horm. Behav. 60, 275-283. doi:10.1016/j.yhbeh.2011.06.001

Dunlap, K.D., Wingfield, J.C., 1995. External and internal influences on indices of physiological stress. I. Seasonal and population variation in adrenocortical secretion of free-living lizards, Sceloporus occidentalis . J. Exp. Zool. 271, 36-46. doi:10.1002/jez.1402710105

Garstka, W.R., Camazine, B., Crews, D., 1982. Interactions of behavior and physiology during the annual reproductive cycle of the red-sided garter snake (Thamnophis sirtalis parietalis). Herpetologica 104-123.

Gregory, P.T., 2006. Influence of income and capital on reproduction in a viviparous snake: direct and indirect effects. J. Zool. 270, 414-419. doi:10.1111/j.14697998.2006.00149.x

Gregory, P.T., 1973. Life history parameters of a population of red-sided garter snakes (Thamnophis sirtalis parietalis) adapted to a rigorous and fluctuating environment. The University of Manitoba, Winnipeg, MB.

Halpert, A.P., Garstka, W.R., Crews, D., 1982. Sperm transport and storage and its relation to the annual sexual cycle of the female red-sided garter snake (Thamnophis sirtalis parietalis). J. Morphol. 174, 149-159. doi:10.1002/jmor.1051740204

Horton, B.M., Holberton, R.L., 2009. Corticosterone manipulations alter morph-specific nestling provisioning behavior in male white-throated sparrows, Zonotrichia albicollis. Horm. Behav. 56, 510-518. doi:10.1016/j.yhbeh.2009.09.001

Jessop, T.S., 2001. Modulation of the adrenocortical stress response in marine turtles (Cheloniidae): evidence for a hormonal tactic maximizing maternal reproductive investment. J. Zool. 254, 57-65. doi:10.1017/S0952836901000553

Jessop, T.S., Knapp, R., Whittier, J.M., Limpus, C.J., 2002. Dynamic Endocrine Responses to Stress: Evidence for Energetic Constraints and Status Dependence in Breeding Male Green Turtles. Gen. Comp. Endocrinol. 126, 59-67. doi:10.1006/gcen.2001.7769

Jessop, T.S., Sumner, J.M., Limpus, C.J., Whittier, J.M., 2004. Interplay between plasma hormone profiles, sex and body condition in immature hawksbill turtles (Eretmochelys imbricata) subjected to a capture stress protocol. Comp. Biochem. Physiol. A. Mol. Integr. Physiol. 137, 197-204. doi:10.1016/j.cbpb.2003.09.029

Jessop, T.S., Tucker, A.D., Limpus, C.J., Whittier, J.M., 2003. Interactions between ecology, demography, capture stress, and profiles of corticosterone and glucose in a free-living population of Australian freshwater crocodiles. Gen. Comp. Endocrinol. 132, 161-170. doi:10.1016/S0016-6480(03)00078-9

Kitaysky, A.S., Wingfield, J.C., Piatt, J.F., 1999. Dynamics of food availability, body condition and physiological stress response in breeding Black-legged Kittiwakes. Funct. Ecol. 13, 577-584. doi:10.1046/j.1365-2435.1999.00352.x

Klukowski, M., 2011. Effects of breeding season, testosterone and ACTH on the corticosterone response of free-ranging male fence lizards (Sceloporus undulatus). Gen. Comp. Endocrinol. 173, 295-302. doi:10.1016/j.ygcen.2011.06.006 
Koch, K.A., Wingfield, J.C., Buntin, J.D., 2002. Glucocorticoids and parental hyperphagia in ring doves (Streptopelia risoria). Horm. Behav. 41, 9-21. doi:10.1006/hbeh.2001.1726

Korte, S.M., De Boer, S.F., De Kloet, E.R., Bohus, B., 1995. Anxiolytic-like effects of selective mineralocorticoid and glucocorticoid antagonists on fear-enhanced behavior in the elevated plus-maze. Psychoneuroendocrinology 20, 385-394.

Krause, J.S., McGuigan, M.A., Bishop, V.R., Wingfield, J.C., Meddle, S.L., 2015. Decreases in Mineralocorticoid but not Glucocorticoid Receptor mRNA Expression During the Short Arctic Breeding Season in Free-Living Gambel's White-Crowned Sparrow (Zonotrichia leucophrys gambelii). J. Neuroendocrinol. 27, 66-75. doi:10.1111/jne.12237

Landys, M.M., Piersma, T., Ramenofsky, M., Wingfield, J.C., 2004a. Role of the lowaffinity glucocorticoid receptor in the regulation of behavior and energy metabolism in the migratory red knot Calidris canutus islandica. Physiol. Biochem. Zool. PBZ 77, 658-668. doi:10.1086/420942

Landys, M.M., Ramenofsky, M., Guglielmo, C.G., Wingfield, J.C., 2004b. The lowaffinity glucocorticoid receptor regulates feeding and lipid breakdown in the migratory Gambel's white-crowned sparrow Zonotrichia leucophrys gambelii. J. Exp. Biol. 207, 143-154.

Landys, M.M., Ramenofsky, M., Wingfield, J.C., 2006. Actions of glucocorticoids at a seasonal baseline as compared to stress-related levels in the regulation of periodic life processes. Gen. Comp. Endocrinol. 148, 132-149. doi:10.1016/j.ygcen.2006.02.013

Lu, N.Z., Cidlowski, J.A., 2006. Glucocorticoid receptor isoforms generate transcription specificity. Trends Cell Biol. 16, 301-307. doi:10.1016/j.tcb.2006.04.005

Lupien, S.J., McEwen, B.S., Gunnar, M.R., Heim, C., 2009. Effects of stress throughout the lifespan on the brain, behaviour and cognition. Nat. Rev. Neurosci. 10, 434445. doi:10.1038/nrn2639

Lutterschmidt, D.I., Mason, R.T., 2009. Endocrine mechanisms mediating temperatureinduced reproductive behavior in red-sided garter snakes (Thamnophis sirtalis parietalis). J. Exp. Biol. 212, 3108-3118. doi:10.1242/jeb.033100

Lutterschmidt, D.I., Mason, R.T., 2008. Geographic variation in timekeeping systems among three populations of garter snakes (Thamnophis sirtalis) in a common garden. Physiol. Biochem. Zool. PBZ 81, 810-825. doi:10.1086/589900

Lutterschmidt, D.I., Mason, R.T., 2005. A serotonin receptor antagonist, but not melatonin, modulates hormonal responses to capture stress in two populations of garter snakes (Thamnophis sirtalis parietalis and Thamnophis sirtalis concinnus). Gen. Comp. Endocrinol. 141, 259-270. doi:10.1016/j.ygcen.2005.01.012

Lutterschmidt, W.I., Lutterschmidt, D.I., Mason, R.T., Reinert, H.K., 2009. Seasonal variation in hormonal responses of timber rattlesnakes (Crotalus horridus) to reproductive and environmental stressors. J. Comp. Physiol. [B] 179, 747-757. doi:10.1007/s00360-009-0356-2

Mendonça, M.T., Crews, D., 1996. Effects of ovariectomy and estrogen replacement on attractivity and receptivity in the red-sided garter snake (Thamnophis sirtalis 
parietalis). J. Comp. Physiol. [A] 178, 373-381.

Mizoguchi, K., Ishige, A., Aburada, M., Tabira, T., 2003. Chronic stress attenuates glucocorticoid negative feedback: involvement of the prefrontal cortex and hippocampus. Neuroscience 119, 887-897. doi:10.1016/S0306-4522(03)00105-2

Moore, I.T., Greene, M.J., Mason, R.T., 2001. Environmental and seasonal adaptations of the adrenocortical and gonadal responses to capture stress in two populations of the male garter snake, Thamnophis sirtalis. J. Exp. Zool. 289, 99-108.

Moore, I.T., Jessop, T.S., 2003. Stress, reproduction, and adrenocortical modulation in amphibians and reptiles. Horm. Behav. 43, 39-47.

Moore, I.T., LeMaster, M.P., Mason, R.T., 2000a. Behavioural and hormonal responses to capture stress in the male red-sided garter snake, Thamnophis sirtalis parietalis. Anim. Behav. 59, 529-534. doi:10.1006/anbe.1999.1344

Moore, I.T., Lerner, J.P., Lerner, D.T., Mason, R.T., 2000b. Relationships between annual cycles of testosterone, corticosterone, and body condition in male redspotted garter snakes, Thamnophis sirtalis concinnus. Physiol. Biochem. Zool. PBZ 73, 307-312. doi:10.1086/316748

Müller, C., Jenni-Eiermann, S., Jenni, L., 2011. Heterophils/Lymphocytes-ratio and circulating corticosterone do not indicate the same stress imposed on Eurasian kestrel nestlings. Funct. Ecol. 25, 566-576. doi:10.1111/j.13652435.2010.01816.x

Narayan, E.J., Hero, J.-M., 2013. Repeatability of Baseline Corticosterone and Acute Stress Responses to Capture, and Patterns of Reproductive Hormones in Vitellogenic and Non-Vitellogenic Female Fijian Ground Frog (Platymantis vitiana). J. Exp. Zool. Part Ecol. Genet. Physiol. 319, 471-481. doi:10.1002/jez.1810

O’Donnell, R.P., Shine, R., Mason, R.T., 2004. Seasonal Anorexia in the Male Red-Sided Garter Snake, Thamnophis sirtalis parietalis. Behav. Ecol. Sociobiol. 56, 413419.

Oka, K., Kohno, S., Urushitani, H., Guillette Jr., L.J., Ohta, Y., Iguchi, T., Katsu, Y., 2013. Molecular cloning and characterization of the corticoid receptors from the American alligator. Mol. Cell. Endocrinol. 365, 153-161. doi:10.1016/j.mce.2012.10.014

Ouyang, J.Q., Hau, M., Bonier, F., 2011. Within seasons and among years: When are corticosterone levels repeatable? Horm. Behav. 60, 559-564. doi:10.1016/j.yhbeh.2011.08.004

Pennington, K., Dicker, P., Dunn, M.J., Cotter, D.R., 2008. Proteomic analysis reveals protein changes within layer 2 of the insular cortex in schizophrenia.

PROTEOMICS 8, 5097-5107. doi:10.1002/pmic.200800415

Romero, L.M., 2002. Seasonal changes in plasma glucocorticoid concentrations in freeliving vertebrates. Gen. Comp. Endocrinol. 128, 1-24. doi:10.1016/S00166480(02)00064-3

Romero, L.M., Wikelski, M., 2001. Corticosterone Levels Predict Survival Probabilities of Galápagos Marine Iguanas during El Niño Events. Proc. Natl. Acad. Sci. U. S. A. $98,7366-7370$. 
Ronchi, E., Spencer, R.L., Krey, L.C., McEwen, B.S., 1998. Effects of photoperiod on brain corticosteroid receptors and the stress response in the golden hamster (Mesocricetus auratus). Brain Res. 780, 348-351. doi:10.1016/S00068993(97)01303-6

Sapolsky, R.M., Romero, L.M., Munck, A.U., 2000. How do glucocorticoids influence stress responses? Integrating permissive, suppressive, stimulatory, and preparative actions 1. Endocr. Rev. 21, 55-89.

Schmidt, K.L., Furlonger, A.A., Lapierre, J.M., MacDougall-Shackleton, E.A., MacDougall-Shackleton, S.A., 2012. Regulation of the HPA axis is related to song complexity and measures of phenotypic quality in song sparrows. Horm. Behav. 61, 652-659. doi:10.1016/j.yhbeh.2012.02.027

Schoech, S.J., Rensel, M.A., Bridge, E.S., Boughton, R.K., Wilcoxen, T.E., 2009. Environment, glucocorticoids, and the timing of reproduction. Gen. Comp. Endocrinol. 163, 201-207. doi:10.1016/j.ygcen.2008.09.009

Schreck, C.B., Contreras-Sanchez, W., Fitzpatrick, M.S., 2001. Effects of stress on fish reproduction, gamete quality, and progeny. Aquaculture, Reproductive Biotechnology in Finfish Aquaculture 197, 3-24. doi:10.1016/S00448486(01)00580-4

Sheskin, D.J., 2007. Handbook of Parametric and Nonparametric Statistical Procedures. Chapman \& Hall, Boca Raton, FL.

Smith, G.T., Wingfield, J.C., Veit, R.R., 1994. Adrenocortical Response to Stress in the Common Diving Petrel, Pelecanoides urinatrix. Physiol. Zool. 67, 526-537.

Soares, M.C., Cardoso, S.C., Grutter, A.S., Oliveira, R.F., Bshary, R., 2014. Cortisol mediates cleaner wrasse switch from cooperation to cheating and tactical deception. Horm. Behav. 66, 346-350. doi:10.1016/j.yhbeh.2014.06.010

Spencer, R.L., Kalman, B.A., Cotter, C.S., Deak, T., 2000. Discrimination between changes in glucocorticoid receptor expression and activation in rat brain using western blot analysis. Brain Res. 868, 275-286. doi:10.1016/S00068993(00)02341-6

Tronche, F., Kellendonk, C., Kretz, O., Gass, P., Anlag, K., Orban, P.C., Bock, R., Klein, R., Schütz, G., 1999. Disruption of the glucocorticoid receptor gene in the nervous system results in reduced anxiety. Nat. Genet. 23, 99-103. doi:10.1038/12703

Vijayan, M.M., Raptis, S., Sathiyaa, R., 2003. Cortisol treatment affects glucocorticoid receptor and glucocorticoid-responsive genes in the liver of rainbow trout. Gen. Comp. Endocrinol. 132, 256-263. doi:10.1016/S0016-6480(03)00092-3

Vitousek, M.N., Mitchell, M.A., Romero, L.M., Awerman, J., Wikelski, M., 2010. To breed or not to breed: physiological correlates of reproductive status in a facultatively biennial iguanid. Horm. Behav. 57, 140-146. doi:10.1016/j.yhbeh.2009.09.020

Vitousek, M.N., Romero, L.M., 2013. Stress responsiveness predicts individual variation in mate selectivity. Gen. Comp. Endocrinol. 187, 32-38. doi:10.1016/j.ygcen.2013.03.009

Weaver, S.A., Schaefer, A.L., Dixon, W.T., 2000. Western blotting for detection of 
glucocorticoid receptors in the brain and pituitary gland from adrenal intact pigs. Brain Res. 869, 130-136. doi:10.1016/S0006-8993(00)02358-1

Welberg, L.A.M., Seckl, J.R., Holmes, M.C., 2001. Prenatal glucocorticoid programming of brain corticosteroid receptors and corticotrophin-releasing hormone: possible implications for behaviour. Neuroscience 104, 71-79. doi:10.1016/S03064522(01)00065-3

Whitnall, M.H., 1993. Regulation of the hypothalamic corticotropin-releasing hormone neurosecretory system. Prog. Neurobiol. 40, 573-629. doi:10.1016/03010082(93)90035-Q

Whittier, J.M., Crews, D., 1990. Body Mass and Reproduction in Female Red-Sided Garter Snakes (Thamnophis sirtalis parietalis). Herpetologica 46, 219-226. doi: $10.2307 / 3892907$

Williams, C.T., Kitaysky, A.S., Kettle, A.B., Buck, C.L., 2008. Corticosterone levels of tufted puffins vary with breeding stage, body condition index, and reproductive performance. Gen. Comp. Endocrinol. 158, 29-35. doi:10.1016/j.ygcen.2008.04.018

Wingfield, J.C., Maney, D.L., Breuner, C.W., Jacobs, J.D., Lynn, S., Ramenofsky, M., Richardson, R.D., 1998. Ecological Bases of Hormone-Behavior Interactions: The "Emergency Life History Stage." Am. Zool. 38, 191-206. doi:10.1093/icb/38.1.191

Wingfield, J.C., Romero, L.M., 2001. Adrenocortical responses to stress and their modulation in free-living vertebrates. Compr. Physiol.

Wingfield, J.C., Sapolsky, R.M., 2003. Reproduction and Resistance to Stress: When and How. J. Neuroendocrinol. 15, 711-724. doi:10.1046/j.1365-2826.2003.01033.x

Wingfield, J.C., Vleck, C.M., Moore, M.C., 1992. Seasonal changes of the adrenocortical response to stress in birds of the Sonoran desert. J. Exp. Zool. 264, 419-428. doi:10.1002/jez.1402640407

Woodley, S.K., Moore, M.C., 2002. Plasma corticosterone response to an acute stressor varies according to reproductive condition in female tree lizards (Urosaurus ornatus). Gen. Comp. Endocrinol. 128, 143-148. doi:10.1016/S00166480(02)00068-0

Zar, J.H., 1999. Biostatistical analysis. Prentice Hall Upper Saddle River, NJ.

\section{Chapter 6 References}

Astheimer, L.B., Buttemer, W.A., Wingfield, J.C., 1995. Seasonal and acute changes in adrenocortical responsiveness in an arctic-breeding bird. Horm. Behav. 29, 442457. doi:10.1006/hbeh.1995.1276

Breuner, C.W., Orchinik, M., 2001. Seasonal regulation of membrane and intracellular corticosteroid receptors in the house sparrow brain. J. Neuroendocrinol. 13, 412420.

Busch, D.S., Hayward, L.S., 2009. Stress in a conservation context: A discussion of 
glucocorticoid actions and how levels change with conservation-relevant variables. Biol. Conserv. 142, 2844-2853. doi:10.1016/j.biocon.2009.08.013

Cease, A.J., Lutterschmidt, D.I., Mason, R.T., 2007. Corticosterone and the transition from courtship behavior to dispersal in male red-sided garter snakes (Thamnophis sirtalis parietalis). Gen. Comp. Endocrinol. 150, 124-131.

Cornelius, J.M., Boswell, T., Jenni-Eiermann, S., Breuner, C.W., Ramenofsky, M., 2013. Contributions of endocrinology to the migration life history of birds. Gen. Comp. Endocrinol., 10th International Symposium on Avian Endocrinology 190, 47-60. doi:10.1016/j.ygcen.2013.03.027

Dayger, C.A., Cease, A.J., Lutterschmidt, D.I., 2013. Responses to capture stress and exogenous corticosterone vary with body condition in female red-sided garter snakes (Thamnophis sirtalis parietalis). Horm. Behav. 64, 748-754. doi:10.1016/j.yhbeh.2013.09.003

Dayger, C.A., LeMaster, M.P., Lutterschmidt, D.I., in review. Physiological correlates of reproductive decisions: Relationships among body condition, reproductive status, and the hypothalamus-pituitary-adrenal axis in a reptile. Horm. Behav. In review.

Dayger, C.A., Lutterschmidt, D.I., 2017. Patterns of stress responses shift during seasonal life-history transitions: an analysis comparing baseline, maximal and integrated corticosterone in female red-sided garter snakes (Thamnophis sirtalis parietalis). Gen. Comp. Endocrinol. doi:10.1016/j.ygcen.2017.03.008

Dayger, C.A., Lutterschmidt, D.I., 2016. Seasonal and sex differences in responsiveness to adrenocorticotropic hormone contribute to stress response plasticity in redsided garter snakes (Thamnophis sirtalis parietalis). J. Exp. Biol. jeb.130450. doi:10.1242/jeb.130450

Dunlap, K.D., Wingfield, J.C., 1995. External and internal influences on indices of physiological stress. I. Seasonal and population variation in adrenocortical secretion of free-living lizards, Sceloporus occidentalis . J. Exp. Zool. 271, 36-46. doi:10.1002/jez.1402710105

Francis, C.M., Cooke, F., 1986. Differential Timing of Spring Migration in Wood Warblers (Parulinae). The Auk 103, 548-556.

Gore, A.C., 2002. GnRH: the master molecule of reproduction. Springer Science \& Business Media.

Gregory, P.T., 1974. Patterns of spring emergence of the red-sided garter snake (Thamnophis sirtalis parietalis) in the Interlake region of Manitoba. Can. J. Zool. 52, 1063-1069. doi:10.1139/z74-141

Heath, J.A., Dufty, J., 1998. Body condition and the adrenal stress response in captive American kestrel juveniles. Physiol. Biochem. Zool. 71, 67-73.

Holberton, R.L., 1999. Changes in Patterns of Corticosterone Secretion Concurrent with Migratory Fattening in a Neotropical Migratory Bird. Gen. Comp. Endocrinol. 116, 49-58. doi:10.1006/gcen.1999.7336

Holberton, R.L., Parrish, J.D., Wingfield, J.C., 1996. Modulation of the Adrenocortical Stress Response in Neotropical Migrants during Autumn Migration. The Auk 113, 558-564. doi:10.2307/4088976

Houseknecht, K.L., Baile, C.A., Matteri, R.L., Spurlock, M.E., 1998. The biology of 
leptin: a review. J. Anim. Sci. 76, 1405-1420. doi:10.2527/1998.7651405x

Iwata, M., 1995. Downstream migratory behavior of salmonids and its relationship with cortisol and thyroid hormones: A review. Aquaculture, Application of Endocrinology to Pacific Rim Aquaculture 135, 131-139. doi:10.1016/00448486(95)01000-9

Jakob, E.M., Marshall, S.D., Uetz, G.W., 1996. Estimating Fitness: A Comparison of Body Condition Indices. Oikos 77, 61-67. doi:10.2307/3545585

Jessop, T.S., Knapp, R., Whittier, J.M., Limpus, C.J., 2002. Dynamic Endocrine Responses to Stress: Evidence for Energetic Constraints and Status Dependence in Breeding Male Green Turtles. Gen. Comp. Endocrinol. 126, 59-67. doi:10.1006/gcen.2001.7769

Jønsson, K.A., Tøttrup, A.P., Borregaard, M.K., Keith, S.A., Rahbek, C., Thorup, K., 2016. Tracking Animal Dispersal: From Individual Movement to Community Assembly and Global Range Dynamics. Trends Ecol. Evol. 31, 204-214. doi:10.1016/j.tree.2016.01.003

Ketterson, E.D., Fudickar, A.M., Atwell, J.W., Greives, T.J., 2015. Seasonal timing and population divergence: when to breed, when to migrate. Curr. Opin. Behav. Sci., The integrative study of animal behavior 6, 50-58. doi:10.1016/j.cobeha.2015.09.001

Kitaysky, A.S., Wingfield, J.C., Piatt, J.F., 1999. Dynamics of food availability, body condition and physiological stress response in breeding Black-legged Kittiwakes. Funct. Ecol. 13, 577-584. doi:10.1046/j.1365-2435.1999.00352.x

Klukowski, M., 2011. Effects of breeding season, testosterone and ACTH on the corticosterone response of free-ranging male fence lizards (Sceloporus undulatus). Gen. Comp. Endocrinol. 173, 295-302. doi:10.1016/j.ygcen.2011.06.006

Krohmer, R.W., Grassman, M., Crews, D., 1987. Annual reproductive cycle in the male red-sided garter snake, Thamnophis sirtalis parietalis: field and laboratory studies. Gen. Comp. Endocrinol. 68, 64-75.

Krohmer, R.W., Lutterschmidt, D.I., 2011. Environmental and neuroendocrine control of reproduction in snakes, in: Aldridge, R.D., Seaver, D.M. (Eds.), Reproductive Biology and Phylogeny of Snakes. pp. 289-346.

Landys, M.M., Ramenofsky, M., Wingfield, J.C., 2006. Actions of glucocorticoids at a seasonal baseline as compared to stress-related levels in the regulation of periodic life processes. Gen. Comp. Endocrinol. 148, 132-149. doi:10.1016/j.ygcen.2006.02.013

Landys, M.M., Wingfield, J.C., Ramenofsky, M., 2004. Plasma corticosterone increases during migratory restlessness in the captive white-crowned sparrow Zonotrichia leucophrys gambelli. Horm. Behav. 46, 574-581. doi:10.1016/j.yhbeh.2004.06.006

Landys-Ciannelli, M.M., Ramenofsky, M., Piersma, T., Jukema, J., Group, C.R., Wingfield, J.C., 2002. Baseline and Stress-Induced Plasma Corticosterone during Long-Distance Migration in the Bar-Tailed Godwit, Limosa lapponica. Physiol. Biochem. Zool. 75, 101-110. doi:10.1086/338285 
Lattin, C.R., Bauer, C.M., de Bruijn, R., Michael Romero, L., 2012. Hypothalamuspituitary-adrenal axis activity and the subsequent response to chronic stress differ depending upon life history stage. Gen. Comp. Endocrinol. 178, 494-501. doi:10.1016/j.ygcen.2012.07.013

Lattin, C.R., Breuner, C.W., Michael Romero, L., 2016. Does corticosterone regulate the onset of breeding in free-living birds?: The CORT-Flexibility Hypothesis and six potential mechanisms for priming corticosteroid function. Horm. Behav. 78, 107120. doi:10.1016/j.yhbeh.2015.10.020

Leary, C.J., 2009. Hormones and acoustic communication in anuran amphibians. Integr. Comp. Biol. 49, 452-470. doi:10.1093/icb/icp027

Leary, C.J., Harris, S., 2012. Steroid hormone levels in calling males and males practicing alternative non-calling mating tactics in the green treefrog, Hyla cinerea. Horm. Behav. doi:10.1016/j.yhbeh.2012.11.006

Leary, C.J., Jessop, T.S., Garcia, A.M., Knapp, R., 2004. Steroid hormone profiles and relative body condition of calling and satellite toads: implications for proximate regulation of behavior in anurans. Behav. Ecol. 15, 313-320. doi:10.1093/beheco/arh015

Liebl, A.L., Shimizu, T., Martin, L.B., 2013. Covariation among glucocorticoid regulatory elements varies seasonally in house sparrows. Gen. Comp. Endocrinol. 183C, 32-37. doi:10.1016/j.ygcen.2012.11.021

Lutterschmidt, D., unpublished. Sex or candy? Neuroendocrine regulation of seasonal life-history transitions. Presented at the North American Society for Comparative Endocrinology, Ottawa, Ontario, Canada.

Lutterschmidt, D.I., 2012. Chronobiology of reproduction in garter snakes: Neuroendocrine mechanisms and geographic variation. Gen. Comp. Endocrinol. 176, 448-455. doi:10.1016/j.ygcen.2011.12.015

Lutterschmidt, D.I., LeMaster, M.P., Mason, R.T., 2004. Effects of melatonin on the behavioral and hormonal responses of red-sided garter snakes (Thamnophis sirtalis parietalis) to exogenous corticosterone. Horm. Behav. 46, 692-702. doi:10.1016/j.yhbeh.2004.06.013

Lutterschmidt, D.I., Lucas, A.R., 2015. Trans-seasonal activation of brain GnRH: Mechanisms underlying temperature-induced reproduction., in: Integrative and Comparative Biology. Oxford Univ Press Inc Journals Dept, 2001 Evans Rd, Cary, NC 27513 USA, pp. E115-E115.

Lutterschmidt, D.I., Maine, A.R., 2014. Sex or candy? Neuroendocrine regulation of the seasonal transition from courtship to feeding behavior in male red-sided garter snakes (Thamnophis sirtalis parietalis). Horm. Behav. 66, 120-134. doi:10.1016/j.yhbeh.2014.01.011

Lutterschmidt, D.I., Mason, R.T., 2010. Temporally distinct effects of stress and corticosterone on diel melatonin rhythms of red-sided garter snakes (Thamnophis sirtalis). Gen. Comp. Endocrinol. 169, 11-17. doi:10.1016/j.ygcen.2010.06.013

Lutterschmidt, D.I., Mason, R.T., 2009. Endocrine mechanisms mediating temperatureinduced reproductive behavior in red-sided garter snakes (Thamnophis sirtalis parietalis). J. Exp. Biol. 212, 3108-3118. doi:10.1242/jeb.033100 
Lutterschmidt, D.I., Mason, R.T., 2005. A serotonin receptor antagonist, but not melatonin, modulates hormonal responses to capture stress in two populations of garter snakes (Thamnophis sirtalis parietalis and Thamnophis sirtalis concinnus). Gen. Comp. Endocrinol. 141, 259-270. doi:10.1016/j.ygcen.2005.01.012

Maine, A.R., Dayger, C.A., Richards, D.Y., Ramirez, L.M., Lutterschmidt, D., unpublished. Migration to summer feeding grounds is associated with changes in plasma glucocorticoids and glucose in red-sided garter snakes (Thamnophis sirtalis).

Marra, P.P., Hobson, K.A., Holmes, R.T., 1998. Linking Winter and Summer Events in a Migratory Bird by Using Stable-Carbon Isotopes. Science 282, 1884-1886. doi:10.1126/science.282.5395.1884

Moore, F.L., Miller, L.J., 1984. Stress-induced inhibition of sexual behavior: Corticosterone inhibits courtship behaviors of a male amphibian (Taricha granulosa). Horm. Behav. 18, 400-410. doi:10.1016/0018-506X(84)90026-6

Moore, I.T., Greene, M.J., Mason, R.T., 2001. Environmental and seasonal adaptations of the adrenocortical and gonadal responses to capture stress in two populations of the male garter snake, Thamnophis sirtalis. J. Exp. Zool. 289, 99-108.

Moore, I.T., Jessop, T.S., 2003. Stress, reproduction, and adrenocortical modulation in amphibians and reptiles. Horm. Behav. 43, 39-47.

Moore, I.T., LeMaster, M.P., Mason, R.T., 2000a. Behavioural and hormonal responses to capture stress in the male red-sided garter snake, Thamnophis sirtalis parietalis. Anim. Behav. 59, 529-534. doi:10.1006/anbe.1999.1344

Moore, I.T., Lerner, J.P., Lerner, D.T., Mason, R.T., 2000b. Relationships between annual cycles of testosterone, corticosterone, and body condition in male redspotted garter snakes, Thamnophis sirtalis concinnus. Physiol. Biochem. Zool. PBZ 73, 307-312. doi:10.1086/316748

Moore, I.T., Mason, R.T., 2001. Behavioral and hormonal responses to corticosterone in the male red-sided garter snake, Thamnophis sirtalis parietalis. Physiol. Behav. 72, 669-674. doi:10.1016/S0031-9384(01)00413-9

Müller, C., Jenni-Eiermann, S., Jenni, L., 2011. Heterophils/Lymphocytes-ratio and circulating corticosterone do not indicate the same stress imposed on Eurasian kestrel nestlings. Funct. Ecol. 25, 566-576. doi:10.1111/j.13652435.2010.01816.x

Peig, J., Green, A.J., 2009. New perspectives for estimating body condition from mass/length data: the scaled mass index as an alternative method. Oikos 118, 1883-1891. doi:10.1111/j.1600-0706.2009.17643.x

Piersma, T., Reneerkens, J., Ramenofsky, M., 2000. Baseline Corticosterone Peaks in Shorebirds with Maximal Energy Stores for Migration: A General Preparatory Mechanism for Rapid Behavioral and Metabolic Transitions? Gen. Comp. Endocrinol. 120, 118-126. doi:10.1006/gcen.2000.7543

Ramenofsky, M., Wingfield, J.C., 2017. Regulation of complex behavioural transitions: migration to breeding. Anim. Behav. 124, 299-306. doi:10.1016/j.anbehav.2016.09.015

Reiter, R.J., 1993. The melatonin rhythm: both a clock and a calendar. Experientia 49, 
654-664. doi:10.1007/BF01923947

Romero, L.M., 2002. Seasonal changes in plasma glucocorticoid concentrations in freeliving vertebrates. Gen. Comp. Endocrinol. 128, 1-24. doi:10.1016/S00166480(02)00064-3

Salvante, K.G., Williams, T.D., 2003. Effects of corticosterone on the proportion of breeding females, reproductive output and yolk precursor levels. Gen. Comp. Endocrinol. 130, 205-214. doi:10.1016/S0016-6480(02)00637-8

Schmidt, K.L., Furlonger, A.A., Lapierre, J.M., MacDougall-Shackleton, E.A., MacDougall-Shackleton, S.A., 2012. Regulation of the HPA axis is related to song complexity and measures of phenotypic quality in song sparrows. Horm. Behav. 61, 652-659. doi:10.1016/j.yhbeh.2012.02.027

Schoech, S.J., Mumme, R.L., Wingfield, J.C., 1997. Corticosterone, Reproductive Status, and Body Mass in a Cooperative Breeder, the Florida Scrub-Jay (Aphelocoma coerulescens). Physiol. Zool. 70, 68-73. doi:10.2307/30164285

Shine, R., Elphick, M.J., Harlow, P.S., Moore, I.T., LeMaster, M.P., Mason, R.T., 2001. Movements, Mating, and Dispersal of Red-Sided Gartersnakes (Thamnophis sirtalis parietalis) from a Communal Den in Manitoba. Copeia 2001, 82-91.

Shine, R., Mason, R.T., 2005. Do a male garter snake's energy stores limit his reproductive effort? Can. J. Zool. 83, 1265-1270. doi:10.1139/z05-119

Skene, D.J., Pevet, P., Vivien-Roels, B., Masson-Pevet, M., Arendt, J., 1987. Effect of different photoperiods on concentrations of 5-methoxytryptophol and melatonin in the pineal gland of the Syrian hamster. J. Endocrinol. 114, 301-309. doi:10.1677/joe.0.1140301

Smith, G.T., Wingfield, J.C., Veit, R.R., 1994. Adrenocortical Response to Stress in the Common Diving Petrel, Pelecanoides urinatrix. Physiol. Zool. 67, 526-537.

Spitzer, J.C., Morrison, D.G., Wettemann, R.P., Faulkner, L.C., 1995. Reproductive responses and calf birth and weaning weights as affected by body condition at parturition and postpartum weight gain in primiparous beef cows. J. Anim. Sci. 73, 1251-1257. doi:10.2527/1995.7351251x

Vitousek, M.N., Mitchell, M.A., Romero, L.M., Awerman, J., Wikelski, M., 2010. To breed or not to breed: physiological correlates of reproductive status in a facultatively biennial iguanid. Horm. Behav. 57, 140-146. doi:10.1016/j.yhbeh.2009.09.020

Wada, H., 2008. Glucocorticoids: Mediators of vertebrate ontogenetic transitions. Gen. Comp. Endocrinol. 156, 441-453. doi:10.1016/j.ygcen.2008.02.004

Wada, M., Shimizu, T., 2004. Seasonal changes in adrenocortical responses to acute stress in polygynous male bush warblers (Cettia diphone). Gen. Comp.

Endocrinol. 135, 193-200. doi:10.1016/j.ygcen.2003.09.006

Whitnall, M.H., 1993. Regulation of the hypothalamic corticotropin-releasing hormone neurosecretory system. Prog. Neurobiol. 40, 573-629. doi:10.1016/03010082(93)90035-Q

Williams, C.T., Kitaysky, A.S., Kettle, A.B., Buck, C.L., 2008. Corticosterone levels of tufted puffins vary with breeding stage, body condition index, and reproductive performance. Gen. Comp. Endocrinol. 158, 29-35. 
doi:10.1016/j.ygcen.2008.04.018

Wilson, R., Lutterschmidt, D., 2017. Identification of a leptin-like protein in red-sided garter snakes and its effects on reproductive behavior. Integr. Comp. Biol. 57, e188-e456. doi:10.1093/icb/icx001

Wingfield, J.C., Sapolsky, R.M., 2003. Reproduction and Resistance to Stress: When and How. J. Neuroendocrinol. 15, 711-724. doi:10.1046/j.1365-2826.2003.01033.x

Zerani, M., Amabili, F., Mosconi, G., Gobbetti, A., 1991. Effects of captivity stress on plasma steroid levels in the green frog, Rana esculenta, during the annual reproductive cycle. Comp. Biochem. Physiol. A Physiol. 98, 491-496. doi:10.1016/0300-9629(91)90436-G 